Aus der Klinik für Palliativmedizin

(Prof. Dr. med. F. Nauck)

der Medizinischen Fakultät der Universität Göttingen

\title{
Kriterien für die frühe Integration von Palliativmedizin in der Pädiatrie
}

\author{
INAUGURAL - DISSERTATION \\ zur Erlangung des Doktorgrades \\ der Medizinischen Fakultät der \\ Georg-August-Universität zu Göttingen
}

vorgelegt von

Inga Steging

aus

Göttingen

Göttingen 2020 
Dekan:

Referent:

Ko-Referent/ in:

Drittreferent/ in:
Prof. Dr. med. W. Brück

Prof. Dr. med. F. Nauck

Datum der mündlichen Prüfung: 
Hiermit erkläre ich, die Dissertation mit dem Titel "Kriterien für die frühe Integration von Palliativmedizin in der Pädiatrie" eigenständig angefertigt und keine anderen als die von mir angegebenen Quellen und Hilfsmittel verwendet zu haben.

Göttingen, den

(Unterschrift) 


\section{Inhaltsverzeichnis}

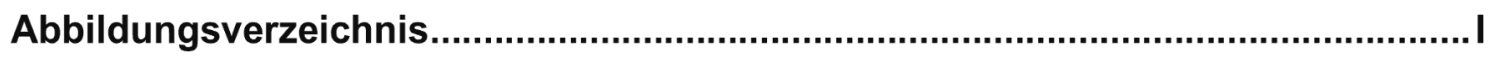

Tabellenverzeichnis ........................................................................................ II

1 Einleitung - Palliativmedizin in der Pädiatrie ................................................. 1

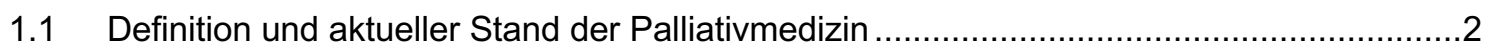

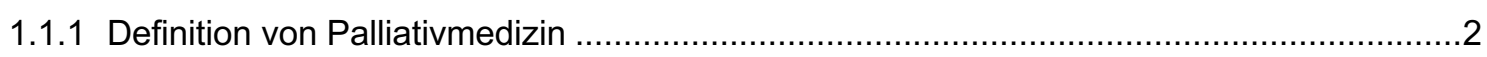

1.1.2 Geschichte und Entwicklung der Palliativmedizin .......................................................

1.1.3 Palliativmedizin im Rahmen des Medizinstudiums und der ärztlichen Weiterbildung.........6

1.1.4 Pädiatrische palliativmedizinische Versorgungsstrukturen im Raum Göttingen ................6

1.1.5 Rechtliche Grundlagen der Begutachtung für SAPV-Leistungen ................................ 8

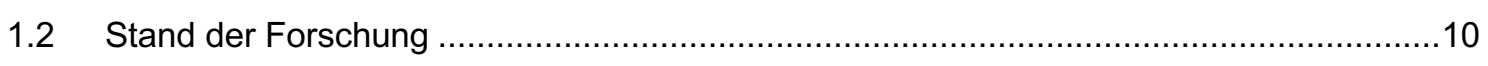

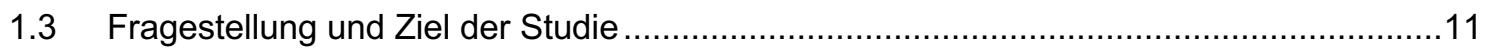

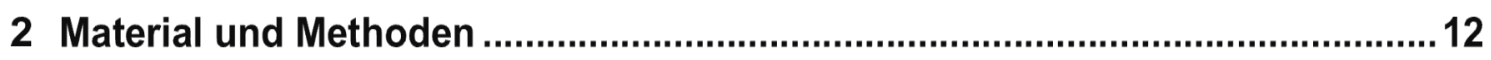

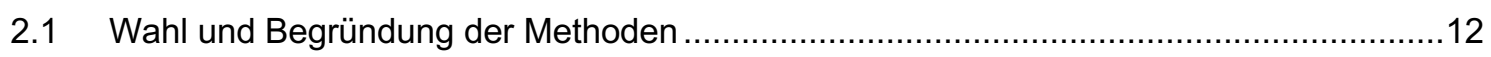

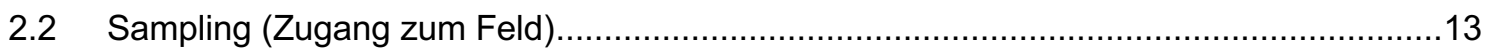

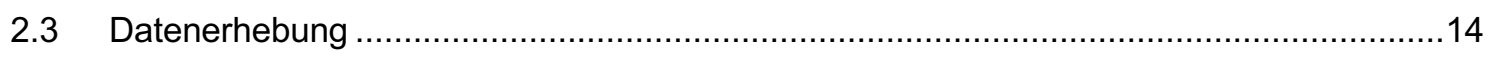

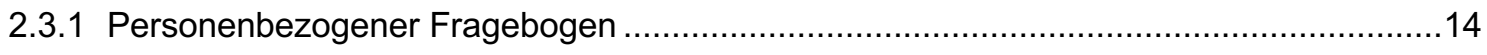



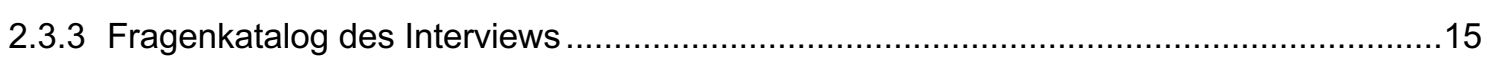

2.3.4 Durchführung der halbstandardisierten Interviews ................................................... 16



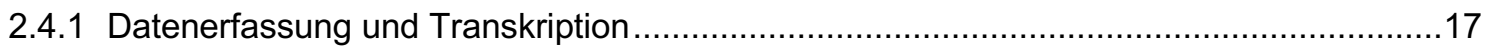

2.4.2 Prinzipien der strukturierten Inhaltsanalyse nach Mayring ......................................... 18

2.4.3 Analyse anhand der strukturierten Inhaltsanalyse nach Mayring .....................................21

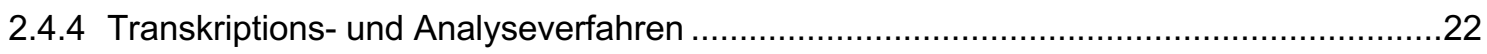

2.5 Datenschutz und Genehmigung durch die Ethikkommission.....................................23

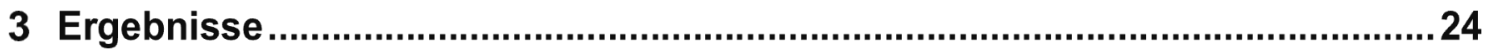

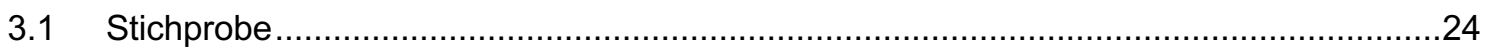

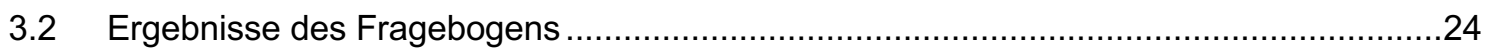

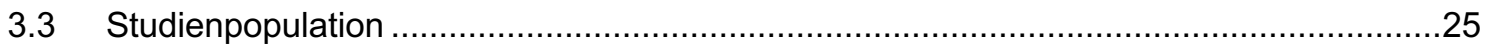

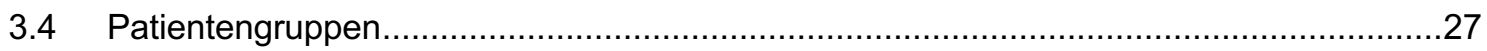




3.6 Ergebnisse der Interviews und Darstellung des Kategoriensystems.....

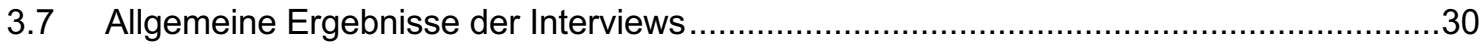

3.8 Ergebnisse der Interviews anhand der Fragestellung und thematischer Kernaussagen 31

3.8.1 Inanspruchnahme / Überweisung an eine spezialisierte ambulante palliativmedizinische Versorgungsstruktur.

3.8.2 Idealer Zeitpunkt der Inanspruchnahme einer spezialisierten ambulanten palliativmedizinischen Versorgungsstruktur....

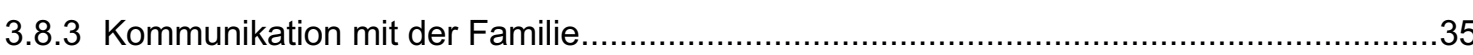

3.9 Weitere Ergebnisse der Interviews unabhängig von der Forschungsfrage ...................36

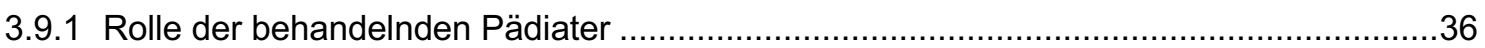

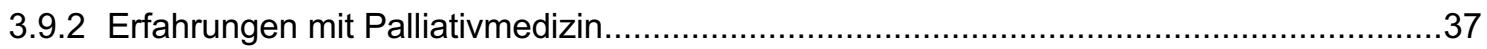

3.9.3 Bedeutung von ambulanter pädiatrischer Versorgung aus Sicht der Pädiater ...............38

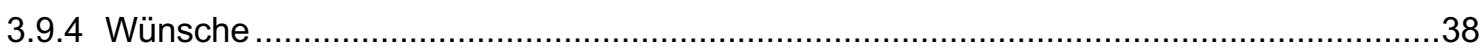

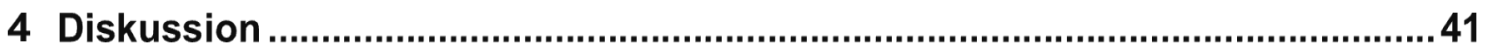

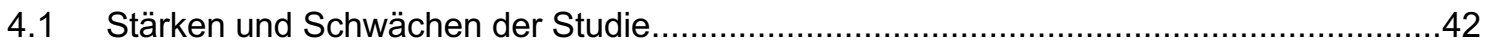

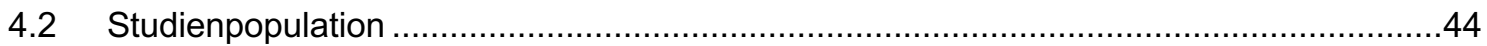

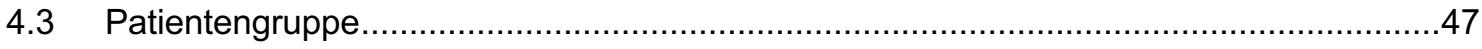

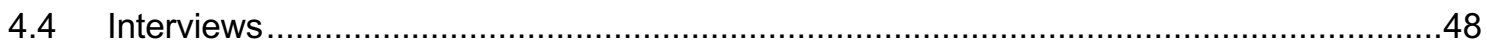

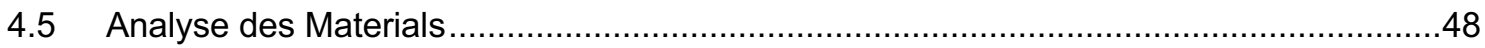

4.6 Verfügbarkeit palliativer pädiatrischer Versorgungsstrukturen in Göttingen Engagement der Pädiater

4.7 Kriterien der Inanspruchnahme einer spezialisierten ambulanten pädiatrischen palliativmedizinischen Versorgungsstruktur.....

4.8 Erfassen eines idealen Zeitpunktes der Inanspruchnahme einer spezialisierten ambulanten palliativmedizinischen Versorgungsstruktur

4.9 Kommunikation mit der Familie.

4.10 Umgang mit der eigenen Rolle als niedergelassener oder stationär tätiger Pädiater im

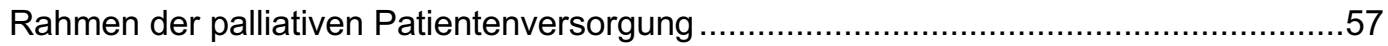

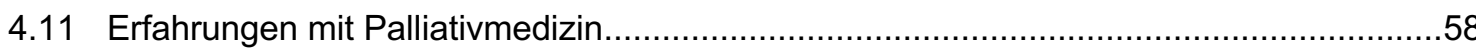

4.12 Bedeutung ambulanter pädiatrischer Palliativversorgung aus Sicht der befragten Pädiater......

4.13 Wünsche .59

4.14 Ausblick....... 


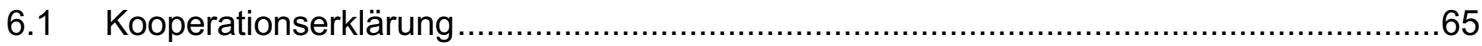

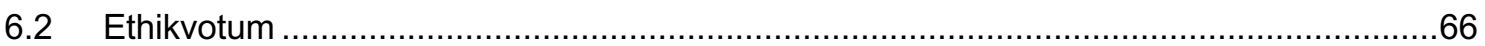

6.3 Information und Aufklärung für die Teilnahme an einem Interview im Rahmen der Studie „Kriterien für die Integration von Palliativmedizin in der Pädiatrie“

6.4 Einverständniserklärung zur Teilnahme an einem Interview im Rahmen der Studie "Kriterien für die Integration von Palliativmedizin in der Pädiatrie"

6.5 Kriterien für die Integration von Palliativmedizin in der Pädiatrie- Befragung niedergelassener oder stationär tätiger Pädiater, Teil 1 Basisdaten

6.6 Kriterien für die Integration von Palliativmedizin in der Pädiatrie- Befragung niedergelassener oder stationär tätiger Pädiater, Teil 2

7 Literaturverzeichnis 


\section{Abbildungsverzeichnis}

Abbildung 1: Ablaufmodell zusammenfassender Inhaltsanalyse,

Qualitative Inhaltsanalyse, Grundlagen und Techniken

(Mayring 2015)

Abbildung 2: Kategorisierungsebenen 


\section{Tabellenverzeichnis}

Tabelle 1: $\quad$ Transkriptionsschema in Anlehnung an BOHNSACK (1993) .................17

Tabelle 2: $\quad$ Übersicht Studienpopulation niedergelassene Pädiater ........................26

Tabelle 3: Übersicht Studienpopulation stationär tätige Pädiater .........................26

Tabelle 4: Kategoriensystem niedergelassene Pädiater .................................29

Tabelle 5: Kategoriensystem stationär tätige Pädiater .....................................30

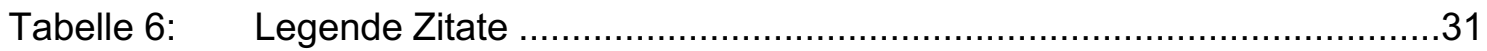




\section{Einleitung - Palliativmedizin in der Pädiatrie}

Bundesweit erkranken jährlich etwa fünfzigtausend Kinder und Jugendliche an einer lebenslimitierenden Erkrankung. Jedes Jahr sterben nahezu eintausend bis eintausendfünfhundert Kinder und Jugendliche in Deutschland an einer unheilbaren, zum Tode führenden Erkrankung (ZERNIKOW 2014). Wenn Kinder an einer lebenslimitierenden Erkrankung leiden, stellt dies sowohl für die Betroffenen als auch ihre Eltern, Geschwister und ihr weiteres soziales Umfeld eine schwere Belastung dar. Die Patienten und ihre Angehörigen bedürfen nach der Diagnosestellung, insbesondere aber auch in der letzten Lebensphase, einer bedarfsorientierten, umfassenden medizinischen, pflegerischen und psychosozialen Unterstützung (FÜHRER 2011).

Um diese Unterstützung zu erreichen, ist in einigen Fällen ein multiprofessionelles Team von Ärzten ${ }^{1}$, Pflegepersonal und weiteren Berufsgruppen nötig. Ziel einer palliativmedizinischen Betreuung sind der Erhalt und die Verbesserung der Lebensqualität für die Betroffenen, die Linderung von Leid und die professionelle Unterstützung und Begleitung der betroffenen Familie. Seit 1998 entstanden in Deutschland stationäre Kinderhospize, ambulante Kinderhospizdienste, Teams der spezialisierten ambulanten Palliativversorgung von Kindern und zwei Kinderpalliativstationen. Zwei Kinderkliniken richteten darüber hinaus ein oder mehrere Palliativzimmer ein. Ziel aller Einrichtungen der Kinderpalliativversorgung ist eine allumfassende Betreuung und Begleitung unheilbar erkrankter Kinder zu gewährleisten.

2007 wurde der Anspruch auf eine so genannte Spezialisierte Ambulante Palliativversorgung (SAPV) in § 37b SGB V (Fünftes Buch Sozialgesetzbuch) gesetzlich verankert. Die speziellen Belange von lebenslimitierend erkrankten Kindern sollen hierbei besondere Berücksichtigung finden. Die Veröffentlichung einer Studie von TADMOR et al. (2003) zeigte, dass sich eine frühzeitige spezialisierte palliativmedizinische Mitbehandlung pädiatrischer Patienten im ambulanten Bereich in hohem Maße günstig auf die Mobilität, Autonomie und die Lebensqualität der Betroffenen auswirkt. Eine frühe Integration von Palliativmedizin in der Pädiatrie ermöglicht Kindern und deren Familien, Entscheidungen über die Versorgung und Pflege zu treffen, die mit ihren eigenen Werten übereinstimmen (MACK und WOLFE 2006).

\footnotetext{
${ }^{1}$ Aus Gründen der besseren Lesbarkeit wird in dieser Arbeit darauf verzichtet, jeweils die weibliche und die männliche Bezeichnung zu verwenden. Soweit neutrale oder männliche Bezeichnungen verwendet werden, sind darunter jeweils weibliche und männliche Personen zu verstehen.
} 
Es stellt sich die Frage, welche Kriterien dazu beitragen könnten, eine frühe Integration von pädiatrischen Patienten, die an einer lebenslimitierenden und weiter fortschreitenden Krankheit leiden, zu erreichen. Bisher fehlen Kriterien, die eine frühe Integration von Palliativmedizin in der Pädiatrie bekräftigen und die Entscheidung für eine Inanspruchnahme spezialisierter palliativmedizinischer Versorgung erwirken.

\subsection{Definition und aktueller Stand der Palliativmedizin}

\subsubsection{Definition von Palliativmedizin}

Unter Palliativmedizin wird die Behandlung und Betreuung von Patienten mit einer nicht heilbaren, progredienten Erkrankung mit begrenzter Lebenserwartung verstanden. Im Vordergrund steht nicht mehr die Auflehnung gegen die Krankheit selbst, sondern vielmehr ein Leben mit der Erkrankung unter Erhalt bzw. Verbesserung der Lebensqualität. Nach der aktuellen Definition der Weltgesundheitsorganisation (WHO) wird Palliativversorgung verstanden als „Ansatz zur Verbesserung der Lebensqualität von Patienten und ihren Familien, die mit Problemen konfrontiert sind, die mit einer lebensbedrohlichen Erkrankung einhergehen, und zwar durch Vorbeugung und Lindern von Leiden, durch frühzeitiges Erkennen, Einschätzen und Behandeln von Schmerzen sowie anderen belastenden Beschwerden von körperlicher, seelischer oder spiritueller Natur" (WHO 2002a). In einem multiprofessionellen Team aus Pflegenden, Ärzten und Experten weiterer Berufsgruppen, die sich mit der ambulanten und stationären Behandlung von Menschen mit lebenslimitierenden Erkrankungen beschäftigen, erfahren Patienten und deren Angehörige in ihrem Alltag bestmögliche Unterstützung und Hilfe. Dabei steht eine ganzheitliche Betreuung und Begleitung der Patienten und ihrer Angehörigen im Sinne der WHO-Definition im Vordergrund. Die aktuelle Definition der WHO von 2002 beinhaltet auch die Einbindung palliativmedizinischer Versorgungsstrukturen zu einem frühen Zeitpunkt der Behandlung einer lebenslimitierenden Erkrankung: „Palliativmedizin kommt frühzeitig im Krankheitsverlauf zur Anwendung, auch in Verbindung mit anderen Therapien, die eine Lebensverlängerung zum Ziel haben, wie z. B. Chemotherapie oder Bestrahlung, und schließt Untersuchungen ein, die notwendig sind, um belastende Komplikationen besser zu verstehen und zu behandeln. "(WHO 2002b). Zusätzlich verweist die WHO in ihrem Handbuch auf die individuelle Betreuung von Kindern und ihren Familien: „Die Palliativversorgung von Kindern umfasst die aktive Betreuung der körperlichen, geistigen und spirituellen Bedürfnisse des Kindes vom Zeitpunkt der Diagnosestellung an und schließt die Unterstützung der Familie mit ein. Die Versorgenden müssen die körperlichen und psychosozialen Leiden des Kindes erkennen und lindern. Eine effektive Palliativversorgung benötigt einen multidisziplinären Ansatz, der die Familie einbezieht und regionale Unterstützungsangebote nutzbar macht“ (WHO 1998). 
Die Vereinigung International Meeting for Palliative Care in Children, Trento (IMPaCCT), eine Arbeitsgruppe der European Association for Palliative Care (EAPC), adaptiert die WHO-Definition pädiatrischer Palliativversorgung wie folgt:

1. Unter Palliativversorgung von Kindern und Jugendlichen versteht man eine aktive und umfassende Versorgung. Diese berücksichtigt Körper, Seele und Geist des Kindes gleichermaßen und gewährleistet die Unterstützung der gesamten betroffenen Familie.

2. Sie beginnt mit Diagnosestellung und ist unabhängig davon, ob das Kind eine Therapie mit kurativer Zielsetzung erhält.

3. Es ist die Aufgabe der professionellen Helfer, das Ausmaß der physischen, psychischen wie sozialen Belastung des Kindes einzuschätzen und zu minimieren.

4. Wirkungsvolle pädiatrische Palliativversorgung ist nur mit einem breiten multidisziplinären Ansatz möglich, der die Familie und alle öffentlichen Ressourcen mit einbezieht.

5. Sie kann auch bei knappen Ressourcen erfolgreich implementiert werden. Pädiatrische Palliativversorgung kann in Krankenhäusern der höchsten Versorgungsstufe, in den Kommunen und zuhause beim Patienten erbracht werden. (ZERNIKOW 2007)

\subsubsection{Geschichte und Entwicklung der Palliativmedizin}

Die Palliativmedizin gilt als eine junge Disziplin (KLINKHAMMER 2007). Das Wort „palliativ" leitet sich vom lateinischen pallium = Mantel bzw. von palliare $=$ mit dem Mantel bedecken (lindern) ab (PONS 2015). Die Anfänge der modernen Palliativmedizin finden sich in den 1960er und 1970er Jahren. Im Jahre 1967 öffnete die Engländerin Cicely Saunders die Türen des St. Christopher's Hospice in London, eine spezialisierte Einrichtung für schwerst- und sterbenskranke Patienten. Ein häuslicher Besuchsdienst zur Betreuung ambulanter Patienten folgte wenig später (SCHWEIZERISCHE PALLIATIVGESELLSCHAFT 2015). Diese Entwicklung fand in den folgenden Jahren auf der ganzen Welt Anerkennung und acht Jahre später, im Jahre 1975, wurde im Royal Victoria Hospital in Kanada die weltweit erste Palliativstation gegründet. Im St. Louis Hospice in Sheffield, Großbritannien, entstand zur selben Zeit das erste „Day-Care-Center“ für Palliativpatienten (BOENKE 2009).

In Deutschland setzte die Entwicklung erst mit einer gewissen Verzögerung ein. 1983 wurde mit Unterstützung der Deutschen Krebshilfe in der Chirurgischen Universitätsklinik in Köln Deutschlands erste Palliativstation eröffnet (BRAUN 2015). 
1993 entstand ebenfalls an der Universitätsklinik Köln mit Unterstützung der Deutschen Krebshilfe die Dr. Mildred Scheel Akademie für Forschung- und Bildung mit einem umfassenden palliativmedizinischen Fort- und Weiterbildungsangebot (DEUTSCHE KREBSHILFE 2015). 1994 wurde mit dem Ziel der Etablierung der Palliativmedizin die Deutsche Gesellschaft für Palliativmedizin (DGP) gegründet (PALLIATIVPORTAL 2015). Der erste Lehrstuhl für Palliativmedizin in Deutschland entstand 1999 in Bonn (MÜLLER-BUSCH 2014) und es wurde für Fachärzte die Möglichkeit geschaffen, sich im Rahmen einer Zusatzweiterbildung Palliativmedizin zu spezialisieren (PALLIATIVPORTAL 2015). Im Jahre 2007 verabschiedete die Bundesregierung mit dem $\S 37 \mathrm{~b}$ und §132d im SBG $\vee$ den gesetzlichen Anspruch auf ambulante spezialisierte Palliativversorgung. Die ersten SAPV-Verträge mit der Kassenärztlichen Vereinigung und den Krankenkassen konnten 2009 geschlossen werden (PALLIATIVPORTAL 2015). Mit dem Ziel, die Betreuung schwerstkranker Menschen zu verbessern, entstand 2010 die Charta zur Betreuung schwerstkranker und sterbender Menschen in Deutschland. Im Fokus stehen die Förderung der Aus-, Fort- und Weiterbildung, Forschung, Politik, Qualitätsmanagement und die allgemeine Zugänglichkeit der Versorgung für Betroffene (CHARTA BUNDESÄRZTEKAMMER 2015). Heute gibt es in Deutschland etwa 294 Palliativstationen und 1268 ambulante Hospizdienste für erwachsene Patienten (DEUTSCHER HOSPIZund PALLIATIVVERBAND 2016).

Die Idee des weltweit ersten Kinderhospizes entstand in den 1980er Jahren in Großbritannien, als die Nonne und Kinderkrankenschwester Frances Dominica das „HelenHouse" gründete. Die persönliche Freundschaft zu einem an Krebs erkrankten Mädchen waren Ausgangspunkt und Inspiration zur Gründung des Kinderhospizes in Oxford (KLASCHIK 2009). 1990 entstand durch das Engagement von sechs Familien mit lebensverkürzend erkrankten Kindern der Deutsche Kinderhospizverein e.V. (DKHV e.V.), der seither eine Reihe von ambulanten Einrichtungen schaffen konnte (DKHV e.V. 2017). Erstmalig bot sich für betroffene Familien ein Forum des Austausches und der gegenseitigen Unterstützung. Darüber hinaus hat sich der Verein zur Aufgabe gemacht in der Gesellschaft über „Sterben und Tod“ von Kindern zu informieren und die Interessen betroffener Familien zu vertreten und er bietet zahlreiche Seminare und Workshops an (DKHV e.V. 2017). In Deutschland entstand 1998 das erste pädiatrische ambulante Palliativteam in Bonn. Unter der Trägerschaft der Gemeinnützigen Gesellschaft der Franziskanerinnen öffnete 1990 das erste Kinderhospiz Deutschlands, das Kinderhospiz Balthasar in Olpe, seine Pforten (KINDER- und JUGENDHOSPIZ BALTHASAR 2015a). Weitere Hospize entstanden in Hamburg, Berlin, Syke, Wiesbaden, Gelsenkirchen und Düsseldorf (BOENKE 2009). Das Kinderhospiz Olpe versteht sich als ein „Ort zum Leben, Lachen, Sterben und Trauern" (KINDER- und JUGENDHOSPIZ BALTHASAR 2015b). Kinder mit lebenslimitierenden Erkrankungen sollen durch einen Aufenthalt im Hospiz gemeinsam 
mit ihrer Familie Erleichterung, Entlastung und Stärkung erfahren und im Zusammensein mit anderen betroffenen Familien Unterstützung und Austausch finden. Ein weiterer Schwerpunkt der Kinderhospizarbeit liegt in der ambulanten Versorgung lebenslimitierend erkrankter Kinder und deren Familien. Um die betroffenen Familien auch im häuslichen Umfeld angemessen begleiten zu können, werden Kinder mit einer lebenslimitierenden Erkrankung in Deutschland durch etwa 118 ambulante Hospizdienste versorgt (DEUTSCHE GESELLSCHAFT für PALLIATIVMEDIZIN 2015). So kann zum Teil eine Betreuung der Kinder auch im häuslichen Umfeld stattfinden und auch ein Sterben zu Hause in der gewohnten Umgebung ermöglicht werden. Auch über den Tod des Kindes hinaus, in der Trauerphase, erfolgt oftmals eine psychosoziale Begleitung der Familie. Seit 2010 finden lebensbedrohlich erkrankte Kinder und ihre Familien auf der Kinderpalliativstation „Lichtblicke“ des Palliativzentrums der Vestischen Kinder- und Jugendklinik Datteln unter der Leitung von Prof. Dr. med. B. Zernikow professionelle medizinischpflegerische und psychosoziale Unterstützung. „Lichtblicke“, der Name der ersten Kinderpalliativstation in Deutschland, soll deutlich machen, dass es auch in belastenden Situationen, in denen das Dunkel überwiegt, helle Momente gibt, die eine andere Sichtweise auf die Dinge möglich machen. Mit der Eröffnung des Kinderpalliativzentrums des Dr. von Haunerschen Kinderspitals im April 2016 in München entstand in Deutschland noch eine weitere Palliativstation speziell für Kinder. Während des Aufenthaltes eines lebenslimitierend erkrankten Kindes können Eltern und Geschwister während des gesamten Krankenhausaufenthaltes in der Nähe ihres Kindes sein. So besteht für die Familien die Möglichkeit den Aufenthalt zu begleiten und zu unterstützen, bis der Patient wieder in sein häusliches Umfeld entlassen werden kann. Es entstehen Begegnungen mit anderen betroffenen Familien, die Kontakt und Austausch ermöglichen. Die Verbesserung der Lebensqualität für die begrenzte gemeinsame Zeit steht bei der Patientenversorgung stets im Vordergrund. Um eine professionelle Versorgung lebenslimitierend erkrankter Kinder zu gewährleisten, bieten sowohl das Kinderpalliativzentrum Datteln, sowie auch das Kinderpalliativzentrum München, wie auch weitere Initiativen qualifizierte Seminare und Workshops für Mitarbeiter aus den Bereichen Pflege, Medizin und psychosozialer Versorgung an. Im Rahmen der Qualitätssicherung wird z.B. in Datteln die Versorgung durch Projekte der Forschungsabteilung begleitet. Das ehemalige Vodafone Stiftungsinstitut für Kinderschmerztherapie und Pädiatrische Palliativmedizin an der Vestischen Kinder- und Jugendklinik Datteln wurde mit der Erarbeitung des ersten Curriculums „Zusatzweiterbildung Palliativversorgung von Kindern und Jugendlichen für Gesundheits- und Kinderkrankenpfleger, Kinderärzte und psychosoziale Mitarbeiter" beauftragt. Das „Dattelner Curriculum“ bildet inzwischen an sechs weiteren deutschen Instituten die Grundlage des pädagogischen Konzeptes der Weiterbildung (KINDERPALLIA- 
TIVZENTRUM 2017). Die aktive Entwicklung der Palliativmedizin für Kinder und Jugendliche verlangt nach einer Schnittstelle zwischen ambulanter und stationärer Behandlung und bemüht sich aktuell um eine frühzeitige Integration in vorangehende Behandlungskonzepte.

\subsubsection{Palliativmedizin im Rahmen des Medizinstudiums und der ärztli- chen Weiterbildung}

Die Betreuung Sterbender fand als möglicher Prüfungsstoff erstmals 2002 Erwähnung in der Approbationsordnung für Ärzte (ÄAppO) (JANKE-HOPPE 2004). Seit dem Jahr 2009 stellt die Palliativmedizin durch die Gesetzesänderung im August 2009 gemäß $\S$ 27 der Approbationsordnung für Ärzte als Querschnittsbereich 13 (QB 13) ein Pflichtlehrund Prüfungsfach im Medizinstudium dar. Laut Bundesgesundheitsministerium soll somit „eine weitere Grundlage für eine umfassende und kompetente medizinische Versorgung schwerstkranker und sterbender Menschen geschaffen“ werden. Palliativmedizinische Lehre sollte nicht nur Wissen und Fertigkeiten beinhalten, sondern auch eine ärztliche Grundhaltung vermitteln, die der Umgang mit schwerst- und sterbenskranken Patienten und ihrer Angehörigen erfordert (NAUCK et al. 2015). Nach der Weiterbildungsordnung der Bundesärztekammer dient die Zusatzweiterbildung Palliativmedizin dazu, fachliche Kompetenz bei der „Behandlung und Begleitung von Patienten mit einer inkurablen, weit fortgeschrittenen und fortschreitenden Erkrankung unter Einbeziehung des sozialen Umfelds zu erreichen“ und sicherzustellen (WEITERBILDUNGSORDNUNG PALLIATIVMEDIZIN 2003). Die Anerkennung der Palliativmedizin als Zusatzbezeichnung setzt in Deutschland eine Facharztanerkennung in einem klinischen Fach voraus. Als Weiterbildungszeiten werden 12 Monate bei einem Weiterbildungsbefugten gemäß $\S 5$ Abs. 1 Satz 2 oder anteilig ersetzbar durch 120 Stunden Fallseminare einschließlich Supervision sowie ein 40-Stunden-Kurs Weiterbildung gemäß $\S 4$ Abs. 8 in Palliativmedizin gefordert (BOENKE 2009).

\subsubsection{Pädiatrische palliativmedizinische Versorgungsstrukturen im Raum Göttingen}

Für Kinder mit einer lebenslimitierenden Erkrankung besteht in Deutschland seit 2007 der gesetzliche Anspruch auf eine spezialisierte ambulante pädiatrische Palliativversorgung. In Niedersachsen hat sich seither ein flächendeckendes Betreuungsnetzwerk der spezialisierten ambulanten pädiatrischen Palliativversorgung etabliert, welches durch ein zentrales Koordinierungsbüro in Hannover organisiert wird. Multiprofessionelle regionale Teams unterstützen betroffene Familien vor Ort. In Südniedersachsen und den 
angrenzenden Bundesländern finden lebenslimitierend erkrankte Kinder und ihre Familien seit April 2011 in einem Umkreis von etwa einhundert Kilometern Hilfe und Unterstützung durch das Kinderpalliativ-Team Göttingen (KINDERPALLIATIVZENTRUM GÖTTINGEN 2017). Sechsundfünfzig Patienten und ihre Familien wurden von 2011 bis 2016 von den Mitarbeitern der spezialisierten ambulanten Palliativversorgung in Göttingen im Rahmen der SAPV-Versorgung zuhause betreut. Das Kinderpalliativzentrum steht unter der Leitung von Frau Professor Dr. med. J. Gärtner, Direktorin der Klinik für Kinder- und Jugendmedizin, und Herrn Professor Dr. med. F. Nauck, Direktor der Klinik für Palliativmedizin, und bietet eine spezialisierte stationäre und ambulante Palliativversorgung für Kinder, Jugendliche und junge Erwachsene. Ein Team aus Kinderärzten verschiedener Fachrichtungen, Palliativmedizinern aus dem Erwachsenenbereich, Palliativfachkräften aus der Gesundheits- und Kinderkrankenpflege, Physiotherapeuten, sowie Experten verschiedener psychosozialer Berufsgruppen bietet professionelle Hilfe. Weitere Mithilfe erhält das Kinderpalliativzentrum durch die Elternhilfe für das krebskranke Kind Göttingen e.V. und die ehrenamtliche Mitarbeit des Ambulanten Kinderhospizdienstes Göttingen. Die stationäre Versorgung der Patienten erfolgt auf allen Stationen der Kinderklinik. Darüber hinaus kann die Betreuung lebenslimitierend erkrankter Kinder auch in zwei aus Spendenmitteln finanzierten, auf die speziellen Bedürfnisse der jungen Patienten angepassten Kinderpalliativzimmern stattfinden. Damit ist eine Kinderpalliativstation an der Universitätsmedizin Göttingen nicht erforderlich und die lebenslimitiert erkrankten Kinder, Jugendlichen und jungen Erwachsenen können dort auch palliativmedizinisch behandelt werden, wo sie früher bereits stationär betreut wurden. Erwachsene Patienten haben die Möglichkeit auf die Palliativstation der Klinik für Palliativmedizin aufgenommen zu werden. Ein besonderer Schwerpunkt des Kinderpalliativzentrums Göttingen und der Versorgung von lebenslimitierend erkrankten Kindern im Allgemeinen, liegt in der ambulanten Betreuung der Patienten und ihrer Angehörigen. Viele Kinder mit einer lebenslimitierenden Erkrankung und deren Familien wünschen sich so viel wie möglich zuhause in ihrer gewohnten Umgebung sein zu können und soweit wie möglich Anteil am alltäglichen Leben der Familie zu haben. Die spezialisierte ambulante Palliativversorgung (SAPV) für Kinder und Jugendliche des Palliativzentrums Göttingen mit ihrem multiprofessionellen Team bietet den betroffenen Familien rund um die Uhr Unterstützung und Beistand im eigenen Zuhause an und es können so Klinikaufenthalte vermieden werden (KINDERPALLIATIVZENTRUM GÖTTINGEN 2017). Seit 2010 begleitet der ambulante Kinder- und Jugend Hospizdienst (AKHD) Göttingen betroffene Kinder und ihre Familien in einem Umkreis von fünfzig Kilometern um Göttingen. Ehrenamtliche Mitarbeiter unterstützen die Familien im Altag, gehen mit den Kindern spazieren, spielen und sind Ansprechpartner für die Familien (AMBULANTER KINDER- und JUGENDHOSPIZDIENST GÖTTINGEN 2015). Ein Kinderhospiz gibt es in Göttingen 
nicht. In Niedersachsen stehen lebenslimitierend erkrankten Kindern und ihren Familien das Angelika Reichelt Kinder- und Jugendhospiz Joschuas Engelreich in Wilhelmshafen und das Kinderhospiz Löwenherz e.V. in Syke zur Verfügung (BUNDESVERBAND KINDERHOSPIZ e.V. 2017). In Niedersachsen hat die seit Dezember 2008 bestehende Pädiatrische Palliativ AG Niedersachsen ein Konzept der SAPV für Kinder- und Jugendliche erarbeitet. Dieses Konzept sieht eine enge Kooperation zwischen den regionalen Kinderpalliativteams in Niedersachsen und dem Betreuungsnetz schwerkranker Kinder vor (BETREUUNGSNETZ.ORG 2017a). Das Koordinierungsbüro für Niedersachsen befindet sich in Hannover, welches die sechs Regionalteams in Niedersachsen koordiniert (BETREUUNGSNETZ.ORG 2017b). Zu innen gehören neben der SAPV für Kinder- und Jugendliche in Göttingen auch das Regionalteam Braunschweig, Hannover, Oldenburg, Osnabrück und Syke (BETREUUNGSNETZ.ORG 2017c).

\subsubsection{Rechtliche Grundlagen der Begutachtung für SAPV-Leistungen}

Im Mittelpunkt der spezialisierten ambulanten pädiatrischen Palliativversorgung stehen Kinder und Jugendliche mit lebenslimitierenden Erkrankungen. Die Richtlinien des Gemeinsamen Bundesausschusses zur Verordnung von spezialisierter ambulanter Palliativversorgung sind in der Spezialisierten ambulanten Palliativersorgungs-Richtlinie (SAPV-RL) im $\S 1$ bis $\S 8$ geregelt. Ziel der spezialisierten ambulanten Palliativversorgung ist gemäß § 37b SGB V die Erhaltung, Förderung und Verbesserung der Lebensqualität und Selbstbestimmung schwerstkranker Menschen und die Ermöglichung eines menschenwürdigen Lebens bis zum Tod in ihrer vertrauten häuslichen oder familiären Umgebung. Anstelle eines kurativen Ansatzes steht die medizinisch-pflegerische Zielsetzung, Symptome und Leiden einzelfallgerecht zu lindern, im Vordergrund. Im §1 der Richtlinie des Gemeinsamen Bundesausschusses (G-BA) zur Verordnung von spezialisierter ambulanter Palliativversorgung wird diese Zielsetzung aufgegriffen (PALLIATIVVERSORGUNGSRICHTLINIE 2007). Darüber hinaus werden im §1 (G-BA) Angaben gemacht, an welchen Orten oder in welchen Einrichtungen SAPV erbracht werden kann. Dazu zählen beispielsweise der Haushalt des Erkrankten und seiner Familie selbst, Pflegeeinrichtungen oder Hospize, wenn die ärztliche Versorgung im Rahmen der vertragsärztlichen Versorgung nicht ausreicht. Hierbei ist auf die individuellen Bedürfnisse und Wünsche des Patienten und seiner Angehörigen einzugehen und die besonderen Belange von Kindern sind zu berücksichtigen (PALLIATIVVERSORGUNGSRICHTLINIE 2007). Die Anspruchsvoraussetzungen auf SAPV sind in den Richtlinien des Gemeinsamen Bundesausschusses (G-BA) zur Verordnung von spezialisierter ambulanter Palliativversorgung (SAPV-RL) im $\S 2$ festgelegt. 
„§ 2: Versicherte haben Anspruch auf SAPV, wenn

1. sie an einer nicht heilbaren, fortschreitenden und so weit fortgeschrittenen Erkrankung leiden, dass dadurch ihre Lebenserwartung begrenzt ist, und

2. sie unter Berücksichtigung der in $\S 1$ genannten Ziele eine besonders aufwändige Versorgung benötigen, die nach den medizinischen und pflegerischen Erfordernissen auch ambulant oder in stationären Pflegeeinrichtungen erbracht werden kann"

(PALLIATIVVERSORGUNGSRICHTLINIE 2007).

Der $\S 3$ der Richtlinie des Gemeinsamen Bundesausschusses zur Verordnung spezialisierter ambulanter Palliativversorgung definiert die Anforderungen, die an eine Erkrankung gestellt werden, um eine spezialisierte ambulante Palliativversorgung beginnen zu können und erklärt dabei, wann eine Erkrankung als „nicht heilbar“, „fortschreitend“ oder „weit fortgeschritten“ bezeichnet wird. Insbesondere bei Kindern ist laut § 3 Absatz 3 die Voraussetzung für die SAPV als Krisenintervention auch bei einer länger prognostizierten Lebenserwartung erfüllt. In § 4 wird präzisiert, wann der Bedarf nach einer besonders aufwändigen Versorgung besteht. Dabei ist das Vorliegen eines komplexen Symptomgeschehens entscheidend. Ein Symptomgeschehen wird dann als komplex angesehen, wenn mindestens eines der nachstehenden Kriterien erfüllt ist:

- ausgeprägte Schmerzsymptomatik

- ausgeprägte neurologische/psychiatrische/psychische Symptomatik

- ausgeprägte respiratorische/kardiale Symptomatik

- ausgeprägte gastrointestinale Symptomatik

- ausgeprägte ulzerierende/exulzerierende Wunden oder Tumore

- ausgeprägte urogenitale Symptomatik

(PALLIATIVVERSORGUNGSRICHTLINIE 2007)

Besteht bei einem Kind der Bedarf für eine spezialisierte ambulante Palliativversorgung, wird die Erstverordnung auf der Grundlage dieser Richtlinie in der Regel vom behandelnden Kinder- und Jugendmediziner mit Hilfe einer Musterverordnung (Muster 63) spezialisierter ambulanter Palliativversorgung ausgestellt (PALLIATIVVERSORGUNGSRICHTLINIE 2007). 


\subsection{Stand der Forschung}

Intensive Forschungsanstrengungen in verschiedenen Bereichen der Medizin haben maßgeblich zu neuen Erkenntnissen und einem Fortschritt der Medizin beigetragen. Der Bereich der klinischen Forschung in der Palliativmedizin wurde dabei im Vergleich zu anderen Bereichen der Medizin bisher deutlich vernachlässigt (KAASA und RADBRUCH 2008). Das Fehlen von Evidenz wird immer wieder beklagt (NAUCK und RADBRUCH 2012). Um in der Palliativmedizin evidenzbasierte Erkenntnisse in Bereichen der Schmerztherapie, Symptomkontrolle und der psychosozialen Bedürfnisse am Lebensende zu erhalten, bedarf es einer Weiterentwicklung in der Forschung, die ohne Einbeziehung persönlicher Erfahrungen von Patienten, ihrer Angehörigen und des Behandlungsteams nicht möglich wäre. Die Intensivierung der Forschung in der Palliativmedizin muss sich insbesondere auch an subjektiven Bedürfnissen, Prozessen der Entscheidungsfindung, ethischen Fragestellungen und der Umsetzung palliativmedizinischer Versorgung orientieren, weshalb Forschungsmethoden aus Geisteswissenschaften, Psychologie, Soziologie und weiteren Bereichen unverzichtbar sind. Zur Klärung von Forschungsfragen in der Palliativmedizin bieten sich somit auch qualitative Forschungsmethoden an. Die Qualitative Inhaltsanalyse ist eine von vielen qualitativen Methoden, die im Bereich Palliative Care zum Einsatz kommen können (INGLETON und SEYMOR 2001). In der palliativmedizinischen Forschung haben sich qualitative Methoden inzwischen mehr und mehr etabliert. Die in dieser Arbeit angewandte qualitative Inhaltsanalyse nach Mayring zeigt in der Fachliteratur bisher jedoch keine erhebliche Relevanz. Die Suche nach Arbeiten mit der Methode der qualitativen Inhaltsanalyse nach Mayring in der Palliativmedizin ergab in der Datenbank von PubMed im Januar 2017 insgesamt nur sieben Einträge. Sämtliche dieser Studien sind Arbeiten aus Deutschland, die zwischen 2010 und 2017 erschienen sind. Ein einziger Titel widmet sich speziell einer pädiatrisch-palliativmedizinischen Fragestellung. Eine übergeordnete gezielte Suche in der Datenbank PubMed nach Forschungsarbeiten zum Thema Palliativmedizin in der Pädiatrie erbrachte zahlreiche Arbeiten, die sich mit der Einführung und Entwicklung im Bereich der Palliativversorgung von Kindern und Jugendlichen und den Erfahrungen, Bedürfnissen und Eindrücken von Eltern lebenslimitierend erkrankter Kinder und des medizinischen Fachpersonals beschäftigen (BERGSTRAESSER et al. 2015; LOTZ et al. 2016; MONTEROSSO und KRISTJANSON 2008; VERBERNE et al. 2017; WEIDNER et al. 2011). Aus den Studien geht der Wunsch der Familien nach einem würdevollen Umgang mit ihrem Kind und der Familie, einem menschlichen Miteinander, dem Verlangen nach einer Begleitung über den Tod hinaus, sowie die Aufklärung über Angebote palliativmedizinischer Versorgungsstrukturen hervor. Quantitative Forschungsansätze 
dienten bisher häufiger als qualitative Methoden der Untersuchung palliativer Versorgungsstrukturen bezogen auf Symptomkontrolle und Erhalt von Lebensqualität (BYRNE et al. 2011; VOLLENBROICH et al. 2012).

\subsection{Fragestellung und Ziel der Studie}

Diese Studie soll aus der Perspektive von niedergelassenen und stationär tätigen Pädiatern Argumente für die Zuweisung von pädiatrischen Patienten zu einer palliativmedizinischen Mitbehandlung erfassen und gleichzeitig das Bewusstsein für eine frühe Integration von Palliativmedizin in der Pädiatrie schärfen. Durch die Darstellung wichtiger Aspekte aus der Sicht der Zuweisenden sollen Kriterien herausgearbeitet werden, die eine frühzeitige kooperative palliativmedizinische Mitbetreuung von pädiatrischen Palliativpatienten zum Ziel haben und so zu einer umfassenden Versorgung dieser Menschen und ihrer Angehörigen führen.

Im Einzelnen sollen folgende Fragen beantwortet werden:

- Anhand welcher Aspekte entscheiden niedergelassene und stationär tätige Pädiater, ihre Patienten einer palliativmedizinischen Mitbetreuung zuzuweisen.

- Gibt es verallgemeinerbare Voraussetzungen für eine frühe Integration von pädiatrischen Patienten in die Palliativversorgung?

- Welche Einstellung haben niedergelassene und stationär tätige Pädiater gegenüber palliativmedizinischer Mitversorgung?

- Wie unterscheiden sich die Aussagen von niedergelassenen und stationär tätigen Pädiatern mit oder ohne eigene Zusatzbezeichnung Palliativmedizin?

Ziel der Arbeit ist es, mit den gewonnenen Erkenntnissen dieser Studie die Palliativversorgung von Kinder und Jugendlichen weiter verbessern zu können und ihre Notwendigkeit und Bedeutung für betroffene Patienten und deren Familien hervorzuheben. 


\section{Material und Methoden}

\subsection{Wahl und Begründung der Methoden}

Soziale Aspekte der medizinischen Versorgung gewinnen heute zunehmend an Bedeutung. Qualitative Forschungsarbeit, die ihren Schwerpunkt vor allem in Forschungsbereichen der Sozialwissenschaften hat und sich in erster Linie mit einer unmittelbaren Erfassung von sozialen Phänomenen durch Betroffene beschäftigt, rückt auch in der Medizin weiter in den Vordergrund und hat in den letzten Jahrzehnten mehr und mehr an Akzeptanz gewonnen (BARBOUR 2003; HIGGINBOTTOM 2004). Die Forschung über die (frühe) Integration der Palliativmedizin in der Pädiatrie ist ein relativ junges Forschungsfeld, über welches es nur geringe Vorkenntnisse aus früheren Studien gibt. Des Weiteren ist es kaum möglich, die Fragestellung (Kriterien, Einstellungen der Befragten etc.) numerisch-statistisch zu erfassen und zu beantworten. Um einen bislang wenig erforschten Wirklichkeitsbereich zu erschließen, ist eine qualitative Herangehensweise eine empfehlenswerte Möglichkeit sich dem Forschungsgebiet zu nähern (FLICK et al. 2000). Sie ist eine von vielen Methoden, die im Bereich der Palliativmedizin Anwendung finden können (INGLETON und SEYMOUR 2001). Eine qualitative Vorgehensweise ermöglicht ein hohes Maß an methodischer Flexibilität und bietet die Möglichkeit einer größeren Tiefe des Informationsgehaltes, indem die subjektive Sicht der relevanten Gesprächspersonen abgebildet wird. Grundlage dieser Arbeit ist die qualitative Befragung der Gesprächspartner in Form von semistrukturierten Interviews. Mit Hilfe eines im Vorfeld erstellten Interviewleitfadens, der konkrete Fragen zum Forschungsthema beinhaltet, konnte sichergestellt werden, dass der Inhalt der Berichte für die Fragestellung der Arbeit von Bedeutung ist. Der Leitfaden dient bei der Erhebung der qualitativen, verbalen Daten als „roter Faden“ und bietet die Möglichkeit der Rahmung und Fokussierung der Daten. Er ermöglicht ihre bessere Vergleichbarkeit und dient der Strukturierung des gesamten Kommunikationsprozesses (MISOCH 2015). Qualitative Inhaltsanalyse zeichnet sich durch eine stark theorie- und regelgeleitete Vorgehensweise aus. Um das Interviewmaterial zu bearbeiten und auszuwerten, fand die semistrukturierte Inhaltsanalyse nach Mayring Verwendung. Das Grundkonzept der qualitativen Inhaltsanalyse nach Mayring besteht darin, Texte systematisch zu analysieren. Das Material wird hierfür schrittweise mit theoriegeleitet am Text entwickelten Kategoriensystemen bearbeitet. (MAYRING 2002). Im Zentrum der qualitativen Auswertung des Textmaterials steht ein Kategoriensystem als Analyseinstrument, mit dessen Hilfe die Gesichtspunkte festgelegt werden, die aus dem Material gewonnen werden sollen. Die Entwicklung des Kategoriensystems kann deduktiv, basierend auf bereits vorhandenem Wissen, oder induktiv, aus dem Text 
heraus, erarbeitet werden. Die Stärke der qualitativen Inhaltsanalyse ist, dass sie ihr Material in Einheiten zerlegt und nacheinander systematisch bearbeitet. Die Regelgeleitetheit macht die Methode der qualitativen Inhaltsanalyse systematisch und intersubjektiv nachvollziehbar (KOHLBACHER 2006). Anwendung findet sie zur Analyse von Problemen, Kulturen und Sachverhalten (MAYRING 2002). Ein inhaltsanalytisches Vorgehen biete sich bei soziologischen, ethischen oder kulturellen Fragestellungen an, wenn es um die Untersuchung und Darstellung von Erfahrungen, Sichtweisen und Erlebnissen geht.

\subsection{Sampling (Zugang zum Feld)}

Unter einem Feld werden alle „natürlichen“ sozialen Handlungsfelder verstanden, die die potentiellen für eine bestimmte Fragestellung relevanten Personen und Personengruppen beinhalten (MISOCH 2015). Im Rahmen der Studie erfolgte die Befragung niedergelassener und stationär tätiger Pädiater in Südniedersachsen im Sinne eines nicht zufälligen Auswahlverfahrens. In Göttingen praktizieren fünfzehn niedergelassene Pädiater und annähernd dreißig stationär tätige Fachärzte für Kinder- und Jugendmedizin. Ziel der qualitativen Arbeit war der Einschluss eines Kollektivs von zehn niedergelassenen und zehn stationär tätigen Pädiatern in Göttingen, um anschließend die Aussagen der niedergelassenen und stationär tätigen Pädiater gegenüberstellen und vergleichen zu können. Die Stichprobe wurde nach den unten genannten Kriterien ausgewählt. Einschlusskriterien der Interviewpartner für die Teilnahme an der Befragung waren:

- Facharztbezeichnung für Pädiatrie, unabhängig vom Zeitpunkt der Facharztprüfung und zusätzlichen Qualifikationen.

- aktuelle Beschäftigung als stationär tätiger oder niedergelassener Pädiater in Göttingen mit oder ohne vorhandene Zusatzbezeichnung bzw. Weiterbildung als Palliativmediziner.

Die jeweils 10 Personen aus der genannten Grundgesamtheit der Pädiater wurden unter dem Gesichtspunkt eines „convinience sampling“ ausgesucht. Durch eine persönliche Vorstellung seitens der Interviewerin kam es zu einer ersten Kontaktaufnahme zwischen den Gesprächspartnern. Dabei wurde den zu befragenden Personen die Thematik sowie der Ablauf des Interviews erläutert und die Bereitschaft zur Mitarbeit erfragt. Ausnahmslos stimmten die konsultierten niedergelassenen und stationär tätigen Pädiater der Teilnahme an der Befragung zu. Anschließend wurde innen ein im Vorfeld auszufüllender Fragebogen zur Erhebung personenbezogener Daten ausgehändigt und ein weiterer Termin für die Durchführung des Interviews vereinbart. 


\subsection{Datenerhebung}

\subsubsection{Personenbezogener Fragebogen}

Der den Interviewpartnern im Vorfeld ausgehändigte Fragebogen diente dazu, verschiedene Variablen bezogen auf den Pädiater selbst (Alter, Geschlecht, Jahr des Universitätsabschlusses, Ausbildung inklusive einer eventuell vorhandenen Weiterbildung in Palliativmedizin), Angaben zu den Patienten des befragten Pädiaters (Altersdurchschnitt, häufige Grunderkrankungen, geschätzte Häufigkeit eines nicht kurativen/ palliativen Behandlungsansatzes) und die Verfügbarkeit von spezialisierter Palliativversorgung (Kinderpalliativzentrum oder niedergelassener Palliativmediziner für Kinder in der Umgebung, Palliativpflegedienste für Kinder in der Umgebung) zu erfassen.

\subsubsection{Interviews}

Eine besondere Form der qualitativen Datenerhebung ist das Interview, welches eine Methode der verbalen Kommunikation darstellt und damit starke Nähe zum Alltagsgespräch aufweist. Sprachgeschichtlich kann der Begriff Interview auf das französische „entrevue“ zurückgeführt werden, welches als „verabredete Zusammenkunft“, „einander kurz sehen“ oder „sich begegnen“ übersetzt werden kann. Ziel ist es, dass sich der Befragte möglichst frei zum Themengegenstand äußert, und dadurch ein Einblick in seine Relevanzstrukturen und Erfahrungshintergründe gewährt (SCHNELL et al. 1995). Die qualitative Inhaltsanalyse bedient sich einer Vielzahl unterschiedlicher Vorgehensweisen, Interviewtechniken und Analyseverfahren, vgl. Helferich (HELFFERICH 2005). Neben Gruppendiskussionen gehören auch Einzelinterviews zu den Interviewformen der qualitativen Forschung. Einzelinterviews zeichnen sich dadurch aus, einen geschützten Rahmen auch bei sensiblen Themen zu bieten (HOPF 2005). Sie stellen für den Interviewer die Möglichkeit dar, sich ausschließlich auf die individuelle Sichtweise des Befragten zu konzentrieren und fördern die Offenheit des Befragten (PRZYBORSKI und WOHLRAB- SAHR 2010). Interviews können zudem in Hinblick auf ihren Strukturierungsgrad differenziert werden. Standardisierte Interviews stellen ein Vorgehen mit bereits vorgegebenen Fragen und Antwortoptionen dar. Semistrukturierte Interviews orientieren sich thematisch an einem Leitfaden. Um eine Vergleichbarkeit der Daten sicherzustellen, müssen alle relevanten Themen des Leitfadens während der Befragung angesprochen werden. Narrative Interviews sind themenfokussierte Interviews, bei denen der individuelle Schwerpunkt dem Interviewpartner überlassen wird. Das Ziel offener Verfahren ist "die lokale Reproduktion von Sinn und Bedeutung" (HONER 2003). Der Vorteil eines Leitfadeninterviews besteht darin sicherzustellen, dass Aspekte die im Fokus des Interesses stehen, angesprochen werden. Der Leitfaden hat also insgesamt 
eher die Funktion einer Gedächtnisstütze und eines Orientierungsrahmens in der allgemeinen Sondierung (LAMNEK 1995). Die Datenerhebung dieser Arbeit erfolgte in Form von halbstandardisierten Einzelinterviews, welche 1988 von Scheele und Groeben zur Rekonstruktion subjektiver Theorien entwickelt wurden (SCHEELE und GROEBEN 1988). Während der Durchführung des Interviews bot der erarbeitete Leitfaden die Grundstruktur der Befragung. Er sorgt für die Vergleichbarkeit der Ergebnisse unterschiedlicher Interviews und dient bei der Befragung gleichzeitig der Orientierung (BORTZ und DÖRING 1995). Je nach Offenheit und Gesprächsfreudigkeit der Befragten unterschied sich die Interviewsituation in Bezug auf die Rolle des Interviewers. Aufgeschlossenen Interviewpartnern wurde im Gesprächsverlauf die Möglichkeit gegeben, alle für sie bedeutenden Aspekte anzusprechen. Anhand des Leitfadens konnten die Interviewpartner wieder zurück zur Fragestellung geführt werden. Im Gegensatz dazu stellt das semistrukturierte Interview auch bei zurückhaltenden Gesprächspartnern durch leitfadengestütztes Nachfragen des Interviewers sicher, dass alle für die Forschungsfrage relevanten Themen beantwortet werden. Das in dieser Studie verwendete semistrukturierte Interview erlaubte es, alle Aspekte des Forschungsgegenstandes herauszuarbeiten und eine Vergleichbarkeit zwischen den geführten Leitfadeninterviews zu gewährleisten. Vorteil der Leitfadeninterviews in dieser Studie war eine Organisation des eigenen Hintergrundwissens und die Fokussierung des Interviews auf forschungsrelevante Themen. Das Material dieser Arbeit wurde anhand von halbstandardisierten Einzelinterviews erhoben. Nach Einarbeitung in die theoretischen Grundlagen der qualitativen Forschung sowie der Interviewtechnik wurde zunächst ein Interviewleitfaden entwickelt. Der Leitfaden stellt das Gerüst der Datenerhebung dar und ermöglicht eine Vergleichbarkeit der Ergebnisse der geführten Interviews (BORTZ und DÖRING 1995). Er dient insgesamt der Funktion einer Gedächtnisstütze und eines Orientierungsrahmens in der allgemeinen Sondierung (LAMNEK 1995a). Als Vorbereitung für die zu führenden Interviews fand gemeinsam mit der Sozialwissenschaftlerin unserer Forschungsgruppe eine Interviewschulung statt. Vor der Verwendung in der Studie wurde der Leitfaden in einem Probeinterview durch die Doktorandin getestet und nach Diskussion möglicher Schwierigkeiten und sinnvoller Ergänzungen in der Forschungsgruppe überarbeitet und modifiziert (BORTZ und DÖRING 1995).

\subsubsection{Fragenkatalog des Interviews}

\section{Erkrankungssituation:}

- Sie haben im Fragebogen folgende palliativmedizinischen Versorgungsstrukturen genannt: 
In welchen Fällen überweisen Sie an diese Strukturen bzw. nehmen sie in Anspruch?

- Bei welchen Erkrankungen und Symptomen im Speziellen würden Sie palliativmedizinische Versorgungsstrukturen hinzuziehen?

- Ist das Hinzuziehen abhängig von der Symptomstärke?

- Gibt es einen bestimmten Zeitpunkt im Krankheitsverlauf, welcher Sie an palliativmedizinische Mitversorgung denken lässt?

- Können Sie einen konkreten bzw. Ihren letzten Fall schildern, bei dem Sie eine Palliativversorgung mit einbezogen haben?

Kommunikation:

- Zu welchem Zeitpunkt thematisieren Sie gegenüber den Eltern bzw. einem Patienten eine mögliche palliativmedizinische Mitversorgung?

Haltung/ Einstellung:

- Wie sollte die Integration von Palliativmedizin in der Pädiatrie aus Ihrer Sicht aussehen?

- Wann wäre für Sie der ideale Zeitpunkt für die Integration von Palliativmedizin?

\section{Erfahrungen:}

- Wie sehen Ihre bisherigen Erfahrungen bezüglich palliativmedizinischer Mitversorgung aus? (Was war die beste, was die schlechteste Erfahrung?)

- Was verstehen Sie unter Palliativmedizinischer pädiatrischer Versorgung?

\subsubsection{Durchführung der halbstandardisierten Interviews}

Nach Begrüßung und Aufklärung der Interviewpartner bezüglich des Umganges mit dem Material sowie der Anonymisierung der erhobenen Daten und nach der Unterzeichnung der Einverständniserklärung, fand die Aufzeichnung des Interviews mit Hilfe eines Aufnahmegerätes statt. Für das Interview gab es im Vorfeld keinen zeitlich festgelegten Rahmen. Der zeitliche Rahmen der Befragung des einzelnen Interviewpartners betrug durchschnittlich zwischen zehn und fünfzehn Minuten. Bei der Befragung wurde vom Interviewer darauf geachtet, dass das Interview in einem möglichst ungestörten Rahmen, in gewohnter Umgebung stattfinden konnte und alle Punkte des Leitfadens im Gespräch Berücksichtigung fanden. Dabei waren eine offene Vorgehensweise und gewisse Modifikationen des Leitfadens möglich. Es galt, von Anfang an eine angenehme, offene und vertrauensvolle Gesprächsatmosphäre zu schaffen, sodass der Interviewte sich frei äußern konnte und sich das Interview nicht lediglich an der Oberfläche sozialer Erwartungen bewegte. Es gibt keine falschen Antworten, sondern nur jene, die authentisch 
sind und jene, die die verzerrt werden durch die Annahme des Interviewten, dass bestimmte Antworten erwartet würden (soziale Erwünschtheit) (MISOCH 2015). Zum Abschluss des Interviews wurde den Gesprächspartnern die Möglichkeit gegeben, eventuelle Fragen zu klären und Raum für Ergänzungen und Vertiefungen geschaffen. Anschließend wurde dem Interviewpartner für die Bereitschaft und Mitarbeit gedankt und die Tonbandaufnahme beendet.

\subsection{Datenauswertung}

\subsubsection{Datenerfassung und Transkription}

Im Anschluss an die Durchführung der Interviews wurde das Tonmaterial wortgetreu und vollständig transkribiert. Transkription leitet sich vom lateinischen transcriptio bzw. transcribere $a b$ und bedeutet in den empirischen Sozialwissenschaften die Verschriftlichung von verbalen oder auch nonverbalen Daten (WIKIPEDIA 2015). Hierbei bildete ein zuvor in Anlehnung an das Transkriptionssystem von Gabriele Rosenthal erstelltes Transkriptionsschema die Grundlage. Abweichungen vom Transkriptionssystem von Gabriele Rosenthal wurden in der Arbeitsgruppe diskutiert und festgelegt. Durch Anwendung eines bestimmten Transkriptionssystems werden die Richtung und die Dimension der Verschriftlichung und damit die Reduktion der Datenkomplexität festgelegt (MISOCH 2015).

Tabelle 1: Transkriptionsschema in Anlehnung an BOHNSACK (1993)

\begin{tabular}{|c|c|}
\hline , & Kurzes Absetzen (vor und nach dem Komma Leerzeichen setzen) \\
\hline (3) & Pause, Anzahl in Sekunden \\
\hline $\mathrm{L}$ & $\begin{array}{l}\text { Beginn einer Überlappung: } \\
\text { IP: Darf ich etwas sagen } \\
\text { I: } \quad \text { L Ja klar }\end{array}$ \\
\hline nein=nein & Schneller Anschluss, Wortverschleifung, Zusammenziehen \\
\hline nein & Betonung \\
\hline NEIN & Laut \\
\hline 'nein' & Leise \\
\hline jaaa & $\begin{array}{l}\text { Dehnung; je mehr vokale aneinandergereiht werden, desto länger } \\
\text { ist die Dehnung }\end{array}$ \\
\hline viellei- & Abbruch (nach dem abgebrochenen Wort Leerzeichen setzen) \\
\hline$(($ laut lachend $))$ & $\begin{array}{l}\text { Kommentar zu nicht verbalen Ereignissen, Veränderung der Intona- } \\
\text { tion (z. B. bei deutlicher Anheben und Senkung der Stimme etc.) }\end{array}$ \\
\hline I & $\begin{array}{l}\text { Einsetzten und Dauer des kommentierten Phänomens z.B. /((lautes } \\
\text { Motorengeräusch)) }\end{array}$ \\
\hline
\end{tabular}




\begin{tabular}{|l|l|}
\hline (doch) & $\begin{array}{l}\text { Unsichere Transkription z. B. bei verbal schwer verständlichen Äu- } \\
\text { ßerungen }\end{array}$ \\
\hline ( ) & $\begin{array}{l}\text { Äußerung ist unverständlich; die Länge der Klammer zeigt in etwa } \\
\text { die Dauer der unverständlichen Äußerung }\end{array}$ \\
\hline$/ / \mathrm{mhm} / /$ & $\begin{array}{l}\text { Hörersignale, „mhm“ der Interviewerin werden ohne Häkchen (L) im } \\
\text { Text des Interviewten notiert } \\
\text { Externe Personen werden mit EP (1,2,3) gekennzeichnet //EP: } \\
\text { mhm// }\end{array}$ \\
\hline d a h e r & $\begin{array}{l}\text { Langsames Sprechen (Leerzeichen zwischen jedem einzelnen } \\
\text { Buchstaben) }\end{array}$ \\
\hline$?$ & Mit angehobener Stimme sprechend \\
\hline
\end{tabular}

\subsubsection{Prinzipien der strukturierten Inhaltsanalyse nach Mayring}

Das Verfahren der qualitativen Inhaltsanalyse nach Mayring dient der Interpretation von sprachlichem Datenmaterial. Durch Abstraktion soll das Textmaterial mit dieser Methode so bearbeitet und reduziert werden, dass die wesentlichen Inhalte des Ausgangstextes erhalten bleiben. Es entsteht eine komprimierte Kopie des Textes, die die Aussage des Grundmateriales exakt wiedergibt (MAYRING 2015).

Das Vorgehen qualitativer Inhaltsanalysen ist regel- und theoriegeleitet und folgt stets einer definierten Systematik. Sie ist eindeutig gegenstandsbezogen, um die Analyse in allen Schritten intersubjektiv nachvollziehbar zu machen (MAYRING 2008). Die qualitative Inhaltsanalyse wird nach Mayring in drei Abschnitte unterteilt. Die Zusammenfassung mit der Analyse des Textes folgt dem Ziel, das Material so zu reduzieren, dass die wesentlichen Inhalte erhalten bleiben. Durch Abstraktion soll ein überschaubarer Corpus geschaffen werden, der immer noch Abbild des Grundmaterials ist. Während der Eplikation wird zu einzelnen fraglichen Textteilen (Begriffen, Sätzen, ...) zusätzliches Material herangetragen, das das Textverständnis erweitert und die Textstellen erläutert, erklärt und ausdeutet. Unter vorher festgelegten Ordnungskriterien werden bei der Strukturierung bestimmte Aspekte aus dem Material herausgefiltert, die dann einen Querschnitt durch das Material liefern sollen (MAYRING 2015).

Das Grundkonzept der strukturierten Inhaltsanalyse nach Mayring stellt ein Kategoriensystem dar, welches deduktiv auf der Grundlage von bereits bestehendem Wissen oder induktiv am vorliegenden Text entwickelt und bearbeitet werden kann (MAYRING 2002). Die Kategorien stellen die Auswertungsaspekte des Materials in Kurzform dar. Sie ermöglichen auch die Nachvollziehbarkeit der Analyse für andere und somit die Intersub- 
jektivität des Vorgehens (MAYRING 2015). Das Konzept der induktiven Kategorienbildung sieht eine zusammenfassende Inhaltsanalyse des Ausgangsmaterials vor, bei dem die gebildeten Kategorien den Kerngedanken des Textes wiedergeben. Nach der Definition eines Abstraktionsniveaus müssen hierfür Auswahlkriterien bestimmt werden, welche die Bildung der einzelnen Kategorien festlegen und abschließend für die Forschungsfrage relevant sind. Als Abstraktionsniveau wird hier eine Ebene im Prozess der Begriffsbildung verstanden. Je höher das Abstraktionsniveau, desto allgemeiner ist der Begriff (ONLINE LEXIKON für PSYCHOLOGIE und PÄDAGOGIK 2017). Unter Berücksichtigung des Selektionskriteriums wird das Material nach dieser Festlegung Wort für Wort betrachtet, überprüft und zusammengefasst. Die Kategorien werden aus dem Sinngehalt des Textes abgeleitet, bis keine neuen Kategorien mehr gebildet werden können (MAYRING 2008). Im Textdurchlauf werden dabei ausschließlich die Textstellen untersucht, in denen Aussagen zur Fragestellung getroffen wurden. Dabei werden ursprüngliche Textausschnitte zunächst paraphrasiert und unter dem angestrebten Abstraktionsniveau generalisiert. Textstellen mit ähnlicher Bedeutung werden den bereits bestehenden Kategorien zugeordnet (Subsumtion), während Textstellen mit inhaltlich neuem Aussagewert eine eigene Kategorie bilden. Dieses Ablaufmodell der induktiven Kategorienbildung ist in Abbildung 1 dargestellt:

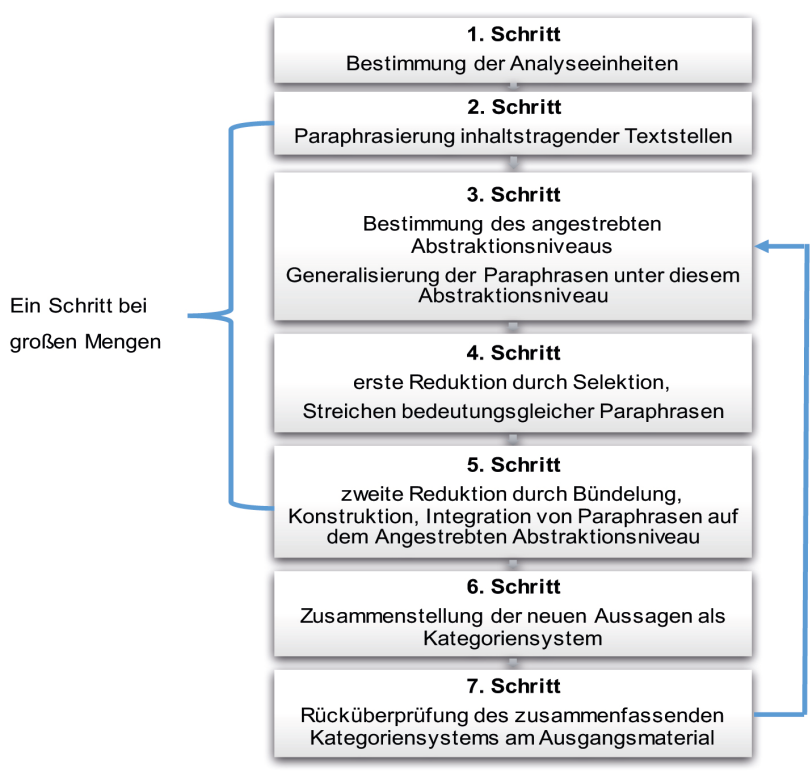

Abbildung 1: Ablaufmodell zusammenfassender Inhaltsanalyse, Qualitative Inhaltsanalyse, Grundlagen und Techniken. Basierend auf MAYRING (2015).

Nachdem auf diese Weise ein großer Teil des Materials durchgearbeitet ist, erfolgt die Überprüfung der erstellten Kategorien bezüglich des Ziels der Analyse und der Einhaltung des Abstraktionsniveaus. Das Ergebnis stellt ein Kategoriensystem zum Thema der 
an den Text gestellten Frage dar. Das Modell der induktiven Kategorienbildung bildet auch die Grundlage für diese Arbeit. Ein „allgemeines Ablaufmodell“ nach Mayring stellt das konkrete praktische Vorgehen dar. Es gliedert die einzelnen Analyseschritte der qualitativen Inhaltsanalyse systematisch und macht es dadurch für andere nachvollziehbar und intersubjektiv überprüfbar (MAYRING 2015).

Im Einzelnen umfasst das Ablaufmodell folgende Schritte (MAYRING 2003):

1. Festlegung des Materials: Das Material dieser Arbeit stellen die Transkripte semistrukturiert geführter Interviews dar, die mit zehn niedergelassenen und zehn stationär tätigen Pädiatern geführt wurden (siehe 5.2. Sampling). Dabei wurden aus den Interviews ausschließlich die Textstellen ausgewählt, die sich auf die Forschungsfrage beziehen.

2. Analyse der Entstehungssituation: Das für diese Arbeit erhobene Datenmaterial soll dazu dienen, aus den Antworten der in den Interviews befragten Pädiater, Kriterien für die Zuweisung von pädiatrischen Patienten zu einer palliativmedizinischen Mitbehandlung herauszuarbeiten. Die Erhebungsgespräche fanden zu diesem Zweck am Arbeitsplatz der Befragten statt (siehe 4 und 5.3.4.).

3. Formale Charakterisierung des Materials: Für die qualitative Inhaltsanalyse betrachtet Mayring es als überaus bedeutsam, die Art des zu analysierenden Materials genau zu bestimmen. Das hier analysierte Datenmaterial ist aus Transkripten semistrukturierter Interviews entstanden, welches nach o.g. Transkriptionsregeln bearbeitet wurde (siehe 5.4.1).

4. Festlegung der Analyserichtung: Die Analyse des Materials richtet sich in dieser Arbeit auf den thematischen Gegenstand des Materials. Dabei fanden auch der emotionale Zustand und die präzise Beurteilung des von den Interviewpartnern Gesagten Berücksichtigung.

5. Theoretische Differenzierung der Fragestellung: Die qualitative Inhaltsanalyse nach Mayring soll nicht alleine stehen, sondern Folge einer fundierten Einordnung in die wissenschaftlichen Erkenntnisse und Diskussionen zum bearbeiteten Themenspektrum sein (siehe 3).

6. Bestimmung der Analysetechnik: An dieser Stelle wird entschieden, welches inhaltsanalytische Verfahren verwendet werden soll. Zur Wahl stehen Zusammenfassung, Explikation und Strukturierung. In der vorliegenden Arbeit wurde die Zusammenfassung als Analyseverfahren gewählt.

7. Definition der Analyseeinheit: In diesem Schritt wird festgelegt, welche Maßeinheit zum Gegenstand der Analyse gemacht werden soll. Dabei stellt die „Kodiereinheit" die kleinste Texteinheit dar, die ausgewertet werden soll. Die größte zu 
interpretierende Einheit bildet die „Kontexteinheit“. Die definierten Analyseeinheiten bilden somit die Elemente, welche für die Bildung von Kategorien Verwendung finden.

8. Durchführung der Materialanalyse: Für die Durchführung der Materialanalyse bietet Mayring drei Analysetechniken an: Zusammenfassung, Explikation und Strukturierung. In der vorliegenden Schrift wurde die Analysetechnik der Zusammenfassung verwendet, die im Folgenden noch weiter erläutert werden soll.

Mit der Analysetechnik der Zusammenfassung wird das Ausgangsmaterial in mehreren Arbeitsschritten gebündelt, gekürzt und auf wesentliche Inhalte reduziert, die sich in der Kategorienbildung wiederspiegeln. Am Ende steht ein „gefülltes“ Kategoriensystem, welches für die Aufbereitung der Ergebnisse zur Verfügung steht.

\subsubsection{Analyse anhand der strukturierten Inhaltsanalyse nach Mayring}

Um das aufgezeichnete Textmaterial anhand der strukturierten Inhaltsanalyse nach Mayring zu bearbeiten, wurden die Interviews mehrfach gehört und die transkribierten Texte von der Doktorandin gelesen. Einzelne transkribierte Textbeispiele wurden in einer monatlich stattfindenden „Forschungswerkstatt" vorgestellt, diskutiert und bearbeitet. Die „Forschungswerkstatt“ bot während des gesamten Zeitraumes der Datenerhebung und Auswertung den Rahmen, sich mit unterschiedlichen Forschungsmethoden vertraut zu machen. Gleichzeitig eröffnete sie die Möglichkeit, erhobene Daten gemeinsam mit anderen Forschern zu diskutieren und die eigene Auswertung zu überprüfen. Gleichzeitig war auf diese Weise ein spezifisches Instrument der qualitativen Inhaltsanalyse, die Intercoder-Reliabilität, gewährleistet. Die Intercoder-Reliabilität kennzeichnet die Übereinstimmungen von Codierungen durch voneinander unabhängige Coder. Abschnitte des Datenmaterials konnten von mehreren Personen codiert, die Ergebnisse verglichen und die Vorgehensweise ggf. modifiziert werden. Um das transkribierte Datenmaterial zu analysieren, wurden basierend auf der Fragestellung und der Literaturanalyse vorab Selektionskriterien und das Abstraktionsniveau bestimmt. Das Vorgehen bestand darin, dass die Daten der zehn Interviews mit den niedergelassenen Pädiatern und das erarbeitete Textmaterial mit den zehn stationär tätigen Pädiatern separat bearbeitet wurden, um am Ende mögliche Unterschiede oder Gemeinsamkeiten herausarbeiten zu können. Es wurde damit begonnen, inhaltstragende Textstellen des Datenmaterials beider Gruppen zu paraphrasieren. Dabei blieben die Paraphrasen zunächst nah am Originaltext und wurden anschließend verallgemeinert. Die vom Ausgangsmaterial erstellten Paraphrasen wurden generalisiert, damit verallgemeinert und auf ein abstrakteres Sprachniveau gebracht. Es ergeben sich somit die in Abbildung 2 dargestellten Kategorisierungsebenen: 

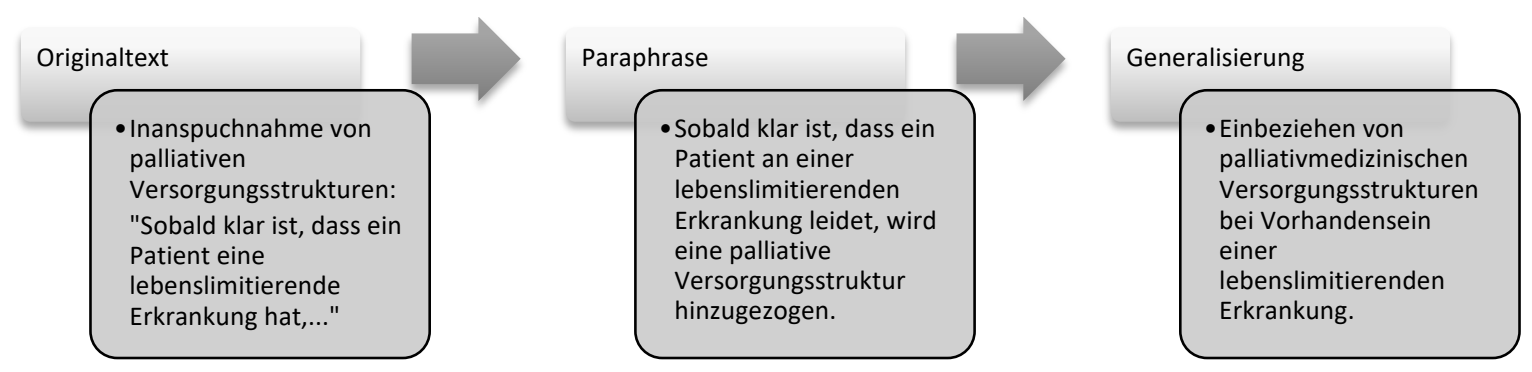

\begin{abstract}
Abbildung 2:
Zusammenfassende qualitative Inhaltsanalyse: Kategorisierungsebenen.

Basierend auf MAYRING (2003).
\end{abstract}

Am Ende dieses Arbeitsschrittes wurden redundante Paraphrasen der beiden Interviewgruppen im Analyseschritt der Reduktion gestrichen bzw. subsumiert und in einem zweiten Schritt Paraphrasen mit gleicher oder ähnlicher Aussage zusammengefasst. Auf diese Weise entstanden einzelne Kategorien oder Codes, die zum endgültigen Kategoriensystem verdichtet wurden. Das Ergebnis bildeten zwei unabhängige Kategoriensysteme. Beide reduzierten Kategoriensysteme wurden nun erneut auf das Ausgangsmaterial angewendet, um ihre Eignung zu überprüfen. War die Reduktion des Materials noch nicht ausreichend, so wurde der gesamte Arbeitsprozess auf einem nächsthöheren Abstraktionsniveau wiederholt. Der Codierprozess endete mit einem Kategoriensystem, welches sich auf die Aussagen niedergelassener Pädiater stützte und ein Kategoriensystem, welches aus dem Datenmaterial der Befragung stationär tätiger Pädiater erarbeitet wurde. Sämtliche relevanten Textstellen der transkribierten Interviews beider Gruppen wurden einer Kategorie zugeordnet, bildeten ein eigenes Kategoriensystem und standen somit für die nachfolgende Auswertung zur Verfügung.

\title{
2.4.4 Transkriptions- und Analyseverfahren
}

Um die verbalen Interviewdaten zu transkribieren, wurde die Audiotranskriptionssoftware f5 (f5; 2014) genutzt. Dies ist eine Spezialsoftware für die Transkription von Video- oder Audioinformationen vorliegender Interviews, mit welcher es möglich ist die Abspielgeschwindigkeit zu steuern, Pausen zu machen oder sich bestimmte Abschnitte noch einmal anzuhören. Für die weitere Auswertung des Transkriptionsmaterials wurde zunächst die Analysesoftware MAXQDA (MAXQDA Version 11; 2014 und MAXQDA Version 12; 2016) verwendet. MAXQDA ist eine Software zur computergestützten qualitativen Daten- und Textanalyse. Sie wird zur Unterstützung von wissenschaftlichen Projekten und Studien angewendet, bei denen Interviews, Texte und Medien wie Bild-, Audio- und Videodateien inhaltlich analysiert werden. Ziel ist, Einblicke 
in das Datenmaterial zu gewinnen, ohne die inhaltliche Interpretation durch die Forschenden vorwegzunehmen ${ }^{2}$. Inhaltliche Kategorien können durch die Software von MAXQDA in einem Kodierbaum mit Codes und Subcodes dargestellt werden. Passagen der importierten Texte können markiert und entsprechenden Codes zugeordnet werden. Mit Hilfe der Software von MAQXDA wurden die angefertigten Transkripte teilweise systematisch codiert und überarbeitet. Die Auswertung der Daten ohne Zuhilfenahme der Analysesoftware erschien jedoch im Hinblick auf den Zeitaufwand und die Genauigkeit bei der Datenauswertung als die präzisere Alternative.

\subsection{Datenschutz und Genehmigung durch die Ethikkommission}

Dieses Projekt ist eine Studie der Klinik für Palliativmedizin (Prof. Dr. med. Friedemann Nauck) der Universitätsmedizin Göttingen in enger Kooperation mit der Klinik für Kinder- und Jugendmedizin (Prof. Dr. med. Jutta Gärtner) der Universitätsmedizin Göttingen, welches der Ethikkommission unter der Antragsnummer 7/7/13 vorgestellt und nach erfolgter Prüfung freigegeben wurde. Sämtliche personenbezogenen Daten wurden verschlüsselt, d. h. die Namen der Interviewpartner wurden durch einen mehrstelligen Buchstaben- und Zahlencode ersetzt. Die erhobenen Daten können nur mittels eines vertraulichen Codes, mit dem das Material pseudonymisiert wurde, einer bestimmten Person zugeordnet werden. Sämtliche Daten und Identifikationsmerkmale werden unter besonderen Schutzvorkehrungen für einen Zeitraum von zehn Jahren getrennt voneinander aufbewahrt und anschließend zentral gelöscht bzw. vernichtet. Die Entschlüsselung ist nur durch die verantwortlichen Personen der Studie möglich. Das einmal gegebene Einverständnis der Interviewpartner kann jederzeit ohne Angabe von Gründen zurückgezogen werden; alle bis zu diesem Zeitpunkt erhobenen Daten würden in diesem Fall gelöscht bzw. vernichtet. In den Interviews wurden keine Patienten befragt, die Befragung wendete sich ausschließlich an stationär tätige und niedergelassene Pädiater.

\footnotetext{
2 www.maxqda.de
} 


\section{Ergebnisse}

\subsection{Stichprobe}

Im Rahmen dieser qualitativen Studie erfolgte die Befragung von niedergelassenen und stationär tätigen Pädiatern im Raum Göttingen. In dieser Studie wurden die Aussagen von zehn stationär tätigen und zehn niedergelassenen Pädiater erfasst. Sämtliche Teilnehmer, die zu diesem Zweck kontaktiert und befragt wurden, waren bereit an der Studie mitzuwirken. Allen Befragten gemein war die Facharztbezeichnung für Kinder- und Jugendmedizin und eine aktuelle Beschäftigung als stationär tätiger oder niedergelassener Pädiater in Göttingen.

\subsection{Ergebnisse des Fragebogens}

Einige Ergebnisse des Fragebogens wurden aufgrund der Verdeutlichung ihrer Wichtigkeit bzw. der Darstellbarkeit einer Rangordnung quantitativ ausgewertet. Diese quantitativen Angaben sind teilweise in Klammern mit einem Summenzeichen eingefügt $(\Sigma \ldots)$. Die Summe benennt in diesem Fall die Anzahl der Befragten, die diese Aussage getroffen haben. Gemeint ist, wenn fünf Pädiater die gleiche Aussage zu einem Punkt im Fragebogen getroffen haben, erscheint in der Klammer $(\Sigma 5)$. In einigen Fällen war es aufgrund der von den Pädiatern gemachten Angaben nicht immer einfach konkrete Summenangaben zu generieren. Hier wurden dann nur sichere Angaben gezählt und aufgeführt. Unproblematisch bei der Bearbeitung des Fragebogens waren Themen, die durch ankreuzen einer oder mehrerer zutreffender Variablen beantwortet werden konnten. Schwierigkeiten bei der Beantwortung ergaben sich insbesondere bei der geforderten Angabe von geschätzten Durchschnittswerten (z.B. geschätzte Häufigkeit eines palliativen Behandlungsansatzes). Vor allem wurden einige der offenen Fragen zum Gegenstand der persönlichen Beschäftigung mit palliativmedizinischen Aspekten sowohl von niedergelassenen, als auch von stationär tätigen Pädiatern nicht beantwortet. Ein möglicher Grund hierfür könnte sein, dass bezogen auf die Gesamtkinderzahl in Deutschland, (etwa 15000000 Kinder) Kinder seltener (etwa 22000 Kinder) an einer lebenslimitierenden Erkrankung leiden (STATISTISCHES BUNDESAMT 2015) und der einzelne Pädiater ohne die Zusatzbezeichnung „Palliativmedizin“ bzw. einer Beschäftigung außerhalb eines ausgewiesenen Zentrums für Kinder- und Jugendmedizin eher selten mit lebenslimitierend erkrankten Kindern in Kontakt kommt. Insgesamt gab es aus Sicht der Befragten keine wesentlichen Probleme mit den Anforderungen des Fragebogens. 


\subsection{Studienpopulation}

In der Studienpopulation der zehn stationär tätigen Pädiater war die Hälfte der Interviewpartner weiblich, die andere Hälfte männlich. Das Durchschnittsalter betrug 41,1 Jahre (32-51 Jahre). Die Approbation hatten die Teilnehmer durchschnittlich vor 15,5 Jahren (7-25 Jahre) erhalten und im Durchschnitt seit 13,1 Jahren (4-23 Jahre) als stationär tätige Pädiater gearbeitet. Im Rahmen ihrer Tätigkeit hatten 50\% der Interviewten eine pädiatrische Schwerpunktbezeichnung (Neurologie $(\Sigma 3)$, Kardiologie ( $\Sigma 2)$, Hämato-

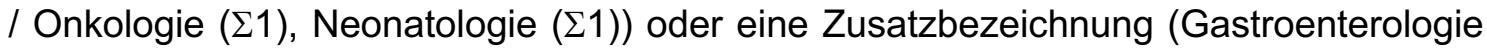
$(\Sigma 1)$, Endokrinologie $(\Sigma 1)$, Intensivmedizin $(\Sigma 1))$ erworben, zwei von innen auch die Zusatzbezeichnung „Palliativmedizin“.

Die Studienpopulation der zehn niedergelassenen Pädiater setzte sich aus sechs männlichen und vier weiblichen Interviewpartnern mit einem Durchschnittsalter von 48 Jahren (39-70 Jahre) zusammen. Die Approbation wurde den Teilnehmern durchschnittlich vor 22 Jahren (14-48 Jahre) erteilt. Vor der Niederlassung hatten sie im Durchschnitt 9,2 Jahre (5-16 Jahre) als stationär tätige Pädiater gearbeitet, die Tätigkeit als niedergelassener Pädiater im ambulanten Bereich betrug durchschnittlich 11 Jahre (2-33 Jahre). $50 \%$ der Befragten hatte eine pädiatrische Schwerpunktbezeichnung (Kardiologie $(\Sigma 3)$, Rheumatologie $(\Sigma 1)$, Pulmologie $(\Sigma 1)$ ) oder eine Zusatzbezeichung (Allergologie $(\Sigma 1)$ ) erworben. Niemand von ihnen hatte an der Zusatzweiterbildung „Palliativversorgung bei Kindern- und Jugendlichen“ mit dem Erwerb der Zusatzbezeichnung „Palliativmedizin“ teilgenommen.

Zur visuellen Veranschaulichung und Gegenüberstellung der Studienpopulation stationär und niedergelassener tätiger Pädiater ist eine Übersicht in der Tabelle 2 bzw. 3 dargestellt. 
Tabelle 2: Übersicht Studienpopulation stationär tätige Pädiater

\begin{tabular}{|c|c|c|c|c|c|c|c|}
\hline Pädiater & $\begin{array}{l}\text { Alter } \\
\text { [Jahre] }\end{array}$ & Geschlecht & Facharzt & $\begin{array}{l}\text { Schwerpunkt-/ Zu- } \\
\text { satzbezeichnung }\end{array}$ & $\begin{array}{l}\text { Zusatzbezeich- } \\
\text { nung Palliativ- } \\
\text { medizin }\end{array}$ & $\begin{array}{l}\text { Stationäre } \\
\text { Tätigkeit } \\
\text { [Jahre] }\end{array}$ & $\begin{array}{l}\text { Ambu- } \\
\text { lante Tä- } \\
\text { tigkeit } \\
\text { [Jahre] }\end{array}$ \\
\hline s_001 & 34 & w & $\sqrt{ }$ & Kardiologie & - & 7 & - \\
\hline s_002 & 32 & w & $\sqrt{ }$ & Endokrinologie & - & 5 & - \\
\hline s_003 & 32 & w & $\sqrt{ }$ & - & - & 4 & - \\
\hline s_004 & 40 & $\mathrm{~m}$ & $\sqrt{ }$ & - & - & 12 & - \\
\hline s_005 & 46 & $\mathrm{~m}$ & $\sqrt{ }$ & Neurologie & - & 18 & - \\
\hline s_006 & 51 & w & $\sqrt{ }$ & Hämato-/ Onkologe & - & 23 & - \\
\hline s_007 & 48 & w & $\sqrt{ }$ & $\begin{array}{l}\text { Kardiologie, } \mathrm{Ne}- \\
\text { onatologie, Inten- } \\
\text { sivmedizin }\end{array}$ & $\sqrt{ }$ & 16 & - \\
\hline s_008 & 44 & $\mathrm{~m}$ & $\sqrt{ }$ & $\begin{array}{l}\text { Gastroenterologie, } \\
\text { Neurologie }\end{array}$ & $\sqrt{ }$ & 16 & - \\
\hline s_009 & 37 & $\mathrm{~m}$ & $\sqrt{ }$ & - & - & 9 & - \\
\hline s_010 & 47 & $\mathrm{~m}$ & $\sqrt{ }$ & Neurologie & - & 17 & - \\
\hline
\end{tabular}

Tabelle 3: $\quad$ Übersicht Studienpopulation niedergelassene Pädiater

\begin{tabular}{|c|c|c|c|c|c|c|c|}
\hline Pädiater & $\begin{array}{l}\text { Alter } \\
\text { [Jahre] }\end{array}$ & Geschlecht & Facharzt & $\begin{array}{l}\text { Schwerpunkt-/ Zu- } \\
\text { satzbezeichnung }\end{array}$ & $\begin{array}{l}\text { Zusatzbezeich- } \\
\text { nung Palliativ- } \\
\text { medizin }\end{array}$ & $\begin{array}{l}\text { Stationäre } \\
\text { Tätigkeit } \\
\text { [Jahre] }\end{array}$ & $\begin{array}{l}\text { Ambulante } \\
\text { Tätigkeit } \\
\text { [Jahre] }\end{array}$ \\
\hline n_001 & 44 & w & $\sqrt{ }$ & keine Angabe & - & 8 & 6 \\
\hline n_002 & 46 & $\mathrm{~m}$ & $\sqrt{ }$ & $\begin{array}{l}\text { Kardiologie/ } \\
\text { Allergologie }\end{array}$ & - & 7 & 11 \\
\hline n_003 & 53 & $\mathrm{~m}$ & $\sqrt{ }$ & - & - & 11 & 13 \\
\hline n_004 & 70 & $\mathrm{~m}$ & $\sqrt{ }$ & - & - & 10 & 33 \\
\hline n_005 & 51 & $\mathrm{~m}$ & $\sqrt{ }$ & Kardiologie & - & 16 & 8 \\
\hline n_006 & 39 & w & $\sqrt{ }$ & Kardiologie & - & 10 & 2 \\
\hline n_007 & 39 & w & $\sqrt{ }$ & - & - & 6 & 5 \\
\hline n_008 & 56 & $\mathrm{~m}$ & $\sqrt{ }$ & - & - & 10 & 21 \\
\hline n_009 & 42 & $\mathrm{~m}$ & $\sqrt{ }$ & Rheumatologie & 一 & 9 & 4 \\
\hline n_010 & 40 & w & $\sqrt{ }$ & Pulmonologie & - & 5 & 7 \\
\hline
\end{tabular}




\subsection{Patientengruppen}

In der Auswertung der Fragebögen gaben acht der stationär tätigen Pädiater im Durchschnitt ein Patientenalter von 6,86 Jahre (5-8 Jahre), einer der Pädiater ein Patientenalter von 0 bis 40 Jahre, ein anderer ein Patientenalter von 0 bis 30 Jahre an. In der Gruppe der stationär tätigen Pädiater sind dies Patienten mit neurologischen Erkrankungen $(\Sigma 6)$ (Epilepsie, Stoffwechselerkrankungen), kardialen Erkrankungen ( $\Sigma 2)$ (Herzfehler), Infektionen $(\Sigma 2)$, onkologischen Erkrankungen $(\Sigma 1)$ (Leukämie, Hirntumoren) und rheumatologische Erkrankungen $(\Sigma 1)$. Ein palliativer Behandlungsansatz besteht nach Angaben der Pädiater durchschnittlich bei 11,72\% (0-50\%) dieser Patienten. Als Diagnose bzw. Art der lebenslimitierenden Erkrankung werden von den stationär tätigen Pädiatern primär neurologische Erkrankungen $(\Sigma 7)$, onkologische Erkrankungen $(\Sigma 3)$ und kardiologische Erkrankungen $(\Sigma 2)$ genannt.

Der Altersdurchschnitt der Patienten im ambulanten Bereich liegt bei 4,36 Jahre (2-10 Jahre). Einer der niedergelassenen Pädiater gab ein Durchschnittsalter der Patienten von $<5$ Jahren an, ein anderer beschreibt seine Patienten vor allem als Patienten des Kleinkindalters. In der Gruppe der niedergelassenen Pädiater werden vorrangig Infekte der oberen Luftwege $(\Sigma 7)$, Gastroenteritiden $(\Sigma 1)$, arterielle Hypertonie $(\Sigma 1)$, Juvenile idiopathische Arthritis $(\Sigma 1)$ als zu behandelnde Grunderkrankungen und Aufgaben in der Vorsorge und Prävention ( $\Sigma 1$ ) (Impfungen) genannt. Im niedergelassenen Bereich ist die Zahl der Patienten mit palliativem Behandlungsansatz deutlich geringer als in der Klinik. Im Durchschnitt sind es 0,31\% (0-0,9\%) aller ambulant betreuten Kinder. Neurologische Erkrankungen $(\Sigma 4)$, onkologische Erkrankungen $(\Sigma 3)$ und Traumafolgen $(\Sigma 1)$ werden von sieben der niedergelassenen Pädiater als lebenslimitierende Diagnosen angegeben, drei machten hierzu keine Angabe, da sie in ihrer Praxis bisher keine Patienten mit lebenslimitierenden Erkrankungen betreuet haben.

\subsection{Palliativversorgung}

Im Raum Göttingen ist die Verfügbarkeit spezialisierter pädiatrischer Palliativversorgung im Gegensatz zu anderen Regionen des Bundesgebietes relativ hoch. Das Kinderpalliativzentrum Göttingen verfügt über zwei stationäre Kinderpalliativbetten und gewährleistet Unterstützung in der spezialisierten ambulanten Palliativversorgung. Daneben bietet der Ambulanter Kinder- und Jugendhospizdienst Göttingen Hilfe für betroffene Familien an.

Die stationär tätigen Pädiater nannten im Umkreis von $50 \mathrm{~km}$ die spezialisierte ambulante pädiatrische Palliativversorgung $(\Sigma 10)$, den pädiatrischen Palliativpflegedienst $(\Sigma 7)$, die pädiatrische Palliativstation $(\Sigma 2)$ und die ambulante Hospizbewegung $(\Sigma 1)$ als 
mögliche Versorgungsstruktur bei Kindern mit lebenslimitierenden Erkrankungen. Die Dauer der eigenen palliativmedizinischen Betreuung pro Patient wurde von sechs Pädiatern durchschnittlich auf 213,4 Tage (5 Tagen bis zu 3 Jahren) geschätzt, vier der Kinderärzte machten hierzu keine Angaben. Vier der stationär tätigen Pädiater sind selber in die spezialisierte ambulante pädiatrische Palliativversorgung eingebunden. Für palliativmedizinische Aspekte nutzen die stationär tätigen Pädiater durchschnittlich 2,3 Stunden in der Woche (0-8 Stunden/Woche). Die angegebene Zeit füllen sie mit Organisation ( $\Sigma 6)$, Gesprächen mit Patienten, Eltern und Kollegen ( $\Sigma 4)$, Versorgung der Patienten zuhause $(\Sigma 4)$, Ausstellen von Rezepten und Hilfsmitteln $(\Sigma 2)$ und mit der Dokumentation. Als angemessen erachten sieben der stationär tätigen Pädiater 7,125 Stunden in der Woche (1-10 Stunden/Woche), um sich um palliativmedizinische Aspekte und die Belange ihrer Patienten zu kümmern. Zusätzlich besteht bei fünf der stationär tätigen Pädiater der Wunsch, sich mit der Verbesserung von Symptomen (Schmerztherapie $(\Sigma 2)$, Schlafstörungen ( $\Sigma 1)$ ), Weiterbildung $(\Sigma 1)$, mehr Hausbesuchen $(\Sigma 1)$, Sterbebegleitung $(\Sigma 1)$, Betreuung der Familien nach dem Tod $(\Sigma 1)$ und der frühen Integration von Palliativmedizin $(\Sigma 1)$ zu beschäftigen.

Die niedergelassenen Pädiater nannten als Strukturen spezialisierter pädiatrischer Palliativversorgung im Umkreis von 50 km die spezialisierte ambulante pädiatrische Palliativversorgung $(\Sigma 10)$, den pädiatrischen Palliativpflegedienst $(\Sigma 8)$, die pädiatrische Palliativstation $(\Sigma 2)$ und die ambulante Hospizbewegung $(\Sigma 2)$ bei Kindern mit lebenslimitierenden Erkrankungen. Zwei der niedergelassenen Pädiater gaben an, selbst keine lebenslimitierend erkrankten Kinder zu betreuen, zwei weitere machten hierzu keine Angaben. Die verbleibenden sechs ambulant tätigen Pädiater machten Angaben zur Dauer der eigenen palliativmedizinischen Betreuung pro Patient von 2 Stunden in der Woche, über mehrere Wochen und Monate, bis hin zu zehn Jahren. Eigenes Engagement in der spezialisierten ambulanten pädiatrischen Palliativversorgung zeigt keiner der befragten niedergelassenen Pädiater. Im Durchschnitt nutzen sieben der Pädiater aus dem ambulanten Bereich 33,57 Minuten in der Woche (0-180 Minuten/Woche) für palliativmedizinische Aspekte, drei von innen beschäftigen sich überhaupt nicht mit diesem Thema. Zu den palliativmedizinischen Aspekten zählen für vier der Befragten Gesprächen mit den Patienten, Eltern und Kollegen ( $\Sigma 4$ ), Organisation ( $(\Sigma 3)$ und die Versorgung der Patienten zuhause $(\Sigma 1)$, sechs der niedergelassenen Pädiater machten hierzu keine Angaben. 50,2 Minuten in der Woche (20-180 Minuten/Woche) werden von den niedergelassenen Pädiatern als angemessener Zeitrahmen betrachtet, um sich mit palliativmedizinischen Aspekten zu befassen. Ein weiteres Interesse, sich mit palliativmedizinischen Aspekten zu befassen besteht bei vier von zehn ambulant tätigen Pädiatern. Hierzu zählen die Betrachtung, Unterstützung und Befriedigung psychosozialer Bedürfnisse der gesamten 
Familie eines erkrankten Patienten mit der Unterstützung durch medikamentöse Versorgung $(\Sigma 2)$, Musiktherapie $(\Sigma 1)$ und Hausbesuche $(\Sigma 1)$.

\subsection{Ergebnisse der Interviews und Darstellung des Kategoriensystems}

Unter Zuhilfenahme eines eigens entwickelten Interviewleitfadens wurden in einem Zeitraum von Juni 2013 bis Februar 2015 zehn stationär tätige und zehn niedergelassene Pädiater befragt. Die durchschnittliche Dauer der mit den stationär tätigen Pädiatern geführten Interviews betrug 10:30 Minuten (07:06-14:12 Minuten), die Länge der mit den niedergelassenen Pädiatern geführten Interviews betrug im Durchschnitt 12:00 Minuten (05:44-17:44 Minuten). Eine Auswertung des Interviewmaterials erfolgte anhand der qualitativen Inhaltsanalyse nach Mayring. In einem Verallgemeinerungsprozess wurden induktiv Kategorien direkt aus dem erhobenen Material abgeleitet. Für die Befragung der stationär tätigen und der niedergelassenen Pädiater entstanden zwei voneinander getrennte Kategoriensysteme mit verschiedenen Codes.

Tabelle 4: Kategoriensystem niedergelassene Pädiater

\begin{tabular}{|c|c|}
\hline 1. & $\begin{array}{l}\text { Lebenslimitierende Erkrankung } \\
\text { - Progredienz der Erkrankung } \\
\text { - Symptomlast } \\
\text { - Häufung von Krankenhausaufenthalten } \\
\text { - Linderung von Leid }\end{array}$ \\
\hline 2. & $\begin{array}{l}\text { Bereitschaft/ Akzeptanz der Familie, Patientenwunsch } \\
\text { - Entscheidungsprozess }\end{array}$ \\
\hline 3. & $\begin{array}{l}\text { Notwendigkeit professioneller Unterstützung } \\
\text { - Überforderung, Belastung, Erschöpfung der Familie } \\
\text { - Unzureichende Versorgungsmöglichkeiten durch andere ambulante } \\
\text { Strukturen }\end{array}$ \\
\hline 4. & Lebensqualität \\
\hline 5. & Eigenes Gefühl \\
\hline 6. & $\begin{array}{l}\text { Rolle des Pädiaters } \\
\text { - Erreichen von Grenzen des behandelnden Pädiaters }\end{array}$ \\
\hline
\end{tabular}


Tabelle 5: Kategoriensystem stationär tätige Pädiater

\begin{tabular}{|c|c|}
\hline 1. & $\begin{array}{l}\text { Lebenslimitierende Erkrankung } \\
\text { - Progredienz der Erkrankung } \\
\text { - Symptomlast } \\
\text { - Häufung von Krankenhausaufenthalten } \\
\text { - Linderung von Leid }\end{array}$ \\
\hline 2. & $\begin{array}{l}\text { Bereitschaft/ Akzeptanz der Familie, Patientenwunsch } \\
\text { - Entscheidungsprozess }\end{array}$ \\
\hline 3. & $\begin{array}{l}\text { Notwendigkeit professioneller Unterstützung } \\
\text { - Überforderung, Belastung, Erschöpfung der Familie } \\
\text { - Unzureichende Versorgungsmöglichkeiten durch andere ambulante } \\
\text { Strukturen }\end{array}$ \\
\hline 4. & Lebensqualität \\
\hline 5. & Eigenes Gefühl \\
\hline
\end{tabular}

\subsection{Allgemeine Ergebnisse der Interviews}

In den Aussagen der Interviews wurden bei allgemeiner Betrachtung der Ergebnisse innerhalb der jeweiligen Bezugsgruppe zu bestimmten Fragestellungen Gemeinsamkeiten deutlich, während sich zu anderen Themen individuelle Unterschiede ergaben. Diese Feststellung gilt zusätzlich im Vergleich der Aussagen der stationär tätigen Pädiater und niedergelassenen Pädiater. Einige Fragen wurden mit konkreteren Angaben beantwortet, andere Bereiche wurden eher allgemein beantwortet. Relativ allgemein blieben die Aussagen zu den Entscheidungsmaßstäben für eine spezialisierte pädiatrische Palliativversorgung.

Übereinstimmung gab es bei den Voraussetzungen für eine Inanspruchnahme von spezialisierter pädiatrischer Palliativversorgung, bei der Einschätzung eines idealen Zeitpunktes für das Hinzuziehen einer palliativen Versorgungsstruktur und dem Ansprechen palliativer Versorgungsmöglichkeiten mit der Familie.

Unterschiede zeigten sich in den Aussagen zur eigenen Rolle von stationär tätigen und niedergelassenen Pädiatern. 


\subsection{Ergebnisse der Interviews anhand der Fragestellung und themati- scher Kernaussagen}

In den nächsten Abschnitten werden die weiteren Ergebnisse der Interviewanalyse dargestellt. Hierzu wurden zum Teil Fragenformulierungen aus den Interviews an den Anfang gestellt. Zur besseren Veranschaulichung und Verdeutlichung der Aussagen wurden inhaltlich interessante Zitate aus den Interviews übernommen. Mit folgenden Abkürzungen und Satzzeichen wurden diese Auszüge wiedergegeben (siehe Tabelle 6):

Tabelle 6: Legende Zitate

\begin{tabular}{|l|l|}
\hline „Beispieltext“ & Zitat aus einem Interview \\
\hline Interviewer: „Beispieltext“ & Gestellte Frage im Interview \\
\hline$[\ldots]$ & $\begin{array}{l}\text { Ausgelassener Interviewabschnitt im Zitat } \\
\text { Anzahl der Befragten, die diese Aussage getroffen ha- } \\
\text { ben. } \quad(\Sigma 2) \text { bedeutet hier: } 2 \text { befragte Personen }\end{array}$ \\
\hline$(\Sigma \ldots)$ & $\begin{array}{l}\text { Stationär tätiger Pädiater } \\
\text { s }\end{array}$ \\
\hline $\mathrm{n}$ & niedergelassener Pädiater \\
\hline
\end{tabular}

\subsubsection{Inanspruchnahme / Überweisung an eine spezialisierte ambulante palliativmedizinische Versorgungsstruktur}

Interviewer: „Im Fragebogen wurden unterschiedliche palliativmedizinischen Versorgungsstrukturen in der Umgebung genannt. In welchen Fällen überweisen Sie an diese Strukturen bzw. nehmen sie in Anspruch?“

Auf diese eingangs gestellte Frage gaben sämtliche der befragten Pädiater $(\Sigma 20)$ an, dass sie beim Vorliegen einer lebenslimitierenden Erkrankung an eine palliativmedizinische Versorgung ihrer Patienten denken. Ein weiterer bedeutender Faktor stellte für beide der ausgewählten Befragungsgruppen der persönliche Patientenwunsch und die Bereitschaft der Eltern bzw. der Familie für das Hinzuziehen einer palliativmedizinischen Versorgungsstruktur dar.

"[...] schon dann, wenn wirklich klar ist, hier liegt ne Erkrankung vor bei dem Kind, die nicht zu kurieren ist, bei der keine Heilung möglich ist. Also schon zu einem relativ frühen Zeitpunkt."(n_004; S.3, 77-79)

„[...] Voraussetzung in aller Regel sind eben lebenslimitierende schwerwiegende Erkrankungen [...]." (s_004; S.1, Z. 47-49) 
„[...] wenn sich abzeichnet, dass es keine weitere erfolgsversprechende Therapie mehr gibt und wenn die Eltern das auch in gewisser Weise akzeptiert haben. [...] wenn die Eltern Palliativmedizin nicht hören wollen, wenn sie sagen wir machen alles so weiter, dann [...] kannste da nicht mit kommen." (s_006; S.1- 2, Z. 31-43)

Für viele der befragten Pädiater stellt der Weg der Familien mit Beginn der Diagnosestellung bis zur Akzeptanz der lebenslimitierenden Erkrankung eines Familienangehörigen eine Entwicklung dar. Häufig, so wurde geschildert, reagieren die Eltern zunächst mit Zurückhaltung auf den Vorschlag einer Unterstützung durch eine spezialisierte palliativmedizinische Versorgungsstruktur.

„[...] immer halt so ganz vorsichtig, [...] es ist ja für die Eltern, ich find das besser, wenn es so ein Prozess ist, d.h. sie können erstmal häufig, sehr häufig Abwehr, nein über Endlichkeit wollen wir jetzt nicht sprechen und ich erwarte auch nicht im ersten Gespräch, dass sie sagen es ist ja ne tolle Idee, das möchten wir gern haben, sondern ich erwarte meistens, dass wir mehrere Kontakte haben werden, bevor jemand sagt o.k., das kann ich mir vorstellen und ich kann mir das auch als Hilfe vorstellen." (s_007; S.3, Z. 79-87)

Daneben war sowohl für die stationär tätigen Pädiater, als auch für die niedergelassenen Pädiater eine erkennbare Notwendigkeit professioneller psychosozialer, pflegerischer und medizinischer Unterstützung (spirituelle Unterstützung wurde nur von einem einzigen Befragten genannt) für den Patienten und die Familie wesentliches Merkmal einer Inanspruchnahme palliativmedizinischer Versorgungsstrukturen. Eine palliative Mitversorgung kommt dann infrage, wenn die familiäre Situation Gefahr läuft zu dekompensieren und die Eltern zuhause überfordert sind. Zu einem Zeitpunkt, an dem die Möglichkeiten einer sonstigen ambulanten Betreuung ausgeschöpft sind, kommt eine spezialisierte palliativmedizinische Versorgung in Betracht. Hierbei begründeten einige der behandelnden Kinderärzte ihre Entscheidung vor allem emotional als Ausdruck ihres eigenen Gefühls.

„Das würde ich in allen Fällen tun, in denen die häusliche Betreuung mit Pflegedienst [...] nicht ausreicht und wo man das Gefühl hat, das auch die Familie selbst nicht in der Lage ist, diese Probleme zuhause auszuhalten oder zu versorgen, Kinder zu versorgen." (n_003; S.1, Z.5-9) 
Von einzelnen niedergelassenen Pädiatern wurde auch das Erreichen eigener Grenzen der Kompetenz in der Betreuung eines lebenslimitierend erkrankten Kindes angesprochen, die an das Hinzuziehen eines professionellen Teams denken lassen.

„[...] das Kind [...] braucht medizinische Unterstützung, die wir als niedergelassene Ärzte nicht leisten können." (n_007; S.1, Z.44-46)

"[...] wenn ich merke auch hier, es geht über das hinaus, was ich leisten kann." (n_001; S.2, Z.45-46)

Ein Ziel der spezialisierten ambulanten Palliativversorgung ist hierbei für alle Befragten der Erhalt und die Verbesserung der Lebensqualität der Patienten sowie eine Besserung von Symptomen, Zufriedenheit und geringere Belastung der Angehörigen.

„[...] wie kann ich die Lebensqualität des Kindes verbessern, wie kann ich dafür sorgen, dass möglichst viel Zeit zuhause bleibt, möglichst viele Aktivitäten noch machen kann, [...] ich finde es immer ganz schön auch für Kinder, die äh, dass die überhaupt mal raus können, weil wenn ich Kinder ganz lange im Krankenhaus einsperre, dann können sie nicht mehr raus, dann wissen sie nicht, was Frühling ist, sie wissen nicht, was Sommer ist und es geht alles an ihnen vorbei. [...] also möglichst viel zuhause zu sein [...]. (s_007; S. 5- 6, Z.174-183)

Interviewer: „Bei welchen Erkrankungen und Symptomen im Speziellen würden Sie palliativmedizinische Versorgungsstrukturen hinzuziehen?"

Allgemein wurden das Vorhandensein und die Progredienz einer lebenslimitierenden Erkrankung als Entscheidungsmerkmal für die Inanspruchnahme einer palliativmedizinischen Versorgungsstruktur genannt. Speziell nannten hier beide der befragten Gruppen neurologische, onkologische und kardiologische Erkrankungen der pädiatrischen Patienten. Bei den genannten Erkrankungsgruppen können die Patienten im Verlauf an unterschiedlichsten Symptomen leiden. Die Gruppe der stationär tätigen Pädiater dachte hierbei maßgebend an Symptome wie Schmerzen ( $\Sigma 7)$, Krampfanfälle $(\Sigma 5)$, pulmonale Probleme ( $\Sigma 4)$, Ernährungsprobleme (z.B. Schluckstörungen) ( $\Sigma 4)$, Bewusstseinsstörungen $(\Sigma 2)$, Angst $(\Sigma 2)$, Ödeme $(\Sigma 2)$ und Juckreiz $(\Sigma 1)$. Von den niedergelassenen Pädiatern wurden bei dieser Frage Atemnot $(\Sigma 6)$, Schmerzen ( $\Sigma 5)$, Ernährungsprobleme (z.B. Schluckstörungen) ( $\Sigma 4)$ und Krampfanfälle $(\Sigma 2)$ genannt. Eine Entscheidung für eine palliativmedizinische Mitversorgung wird laut Aussage einiger Pädiater eher durch die Symptomqualität als durch die Symptomquantität beeinflusst. 
„Eher von der Symptomqualität, nich, also ich denke es gibt Symptome und Erscheinungen im Endstadium einer Krankheit, die sind von Eltern gut zu handeln. Also z.B. dass die Kinder nicht mehr kommunizieren können, ist glaube ich für Eltern eher erträglich, als wenn sie Kinder haben, die z.B. permanent krampfen oder permanent schreien vor Schmerzen, oder ja also ständig unter sich lassen bzw. einfach erbrechen und einfach pflegerisch großen Aufwand betreiben. Nich, also das sind so für mich die Situationen, also die Stärke spielt glaube ich nicht so ne große Rolle, aber das hängt sicherlich auch von den Eltern ab, wie sehr sie unter den Symptomen eben auch mitleiden, ne." (n_008; S.2, Z.39-50)

\subsubsection{Idealer Zeitpunkt der Inanspruchnahme einer spezialisierten ambu- lanten palliativmedizinischen Versorgungsstruktur}

Interviewer: „Gibt es einen bestimmten Zeitpunkt im Krankheitsverlauf, bei dem Sie an palliativmedizinische Mitversorgung denken?"

Ein frühes Angebot palliativer Versorgungsstrukturen bei einer lebenslimitierenden Erkrankung wurde von einer Vielzahl der befragten Pädiater als wünschenswert angesehen. Einen konkreten idealen Zeitpunkt scheint es nicht zu geben. Vielmehr wird der Zeitpunkt in Abhängigkeit der Akzeptanz der Situation durch die Familie und den Verlauf der Erkrankung gesehen. Der Zeitpunkt, bei dem an eine palliative Mitversorgung gedacht wird, scheint somit individuell sehr unterschiedlich zu sein. Angaben zur tatsächlichen Bedeutung von „früh“ wurden von den Befragten nicht konkretisiert. In einer Situation, in der ein Kind lebenslimitierend erkrankt ist und eine Chance auf Heilung nicht mehr besteht, ist es häufig das eigene Gefühl, welches in die Entscheidung über palliative Versorgung mit einfließt.

„[...] wenn ich das Gefühl habe, das hat so'ne gewisse Endlichkeit, dann biete ich das an, auch schon möglichst frühzeitig, [...]." (s_007; S.4, Z. 77-79)

Einige der stationär tätigen Pädiater sahen als realen, aber sicherlich zu späten Zeitpunkt für die Inanspruchnahme einer spezialisierten palliativmedizinischen Versorgungsstruktur das Eintreten einer lebensbedrohlichen Situation.

"Insbesondere dann, wenn der Patient das erste Mal in einer lebensbedrohlichen Situation zu uns gekommen ist. Das ist eigentlich der Zeitpunkt, wo wir dann uns darüber Gedanken machen, ob der Patient eine solche Betreuung hat. Das ist natürlich eigentlich zu spät, aber Norm - so im Tagesgeschäft.“(s_005; S.2, Z. 38-43) 
Als weiteres Merkmal für den Zeitpunkt der Inanspruchnahme einer palliativmedizinischen Versorgungsstruktur wurden eine Häufung stationärer Aufenthalte und der Wunsch der betroffenen Familien, mit dem erkrankten Kind zuhause zu sein, genannt. Ein frühes Einsetzen palliativmedizinischen Versorgungsstrukturen kann aus Sicht der befragten Pädiater dazu beitragen, unnötige stationäre Aufenthalte zu vermeiden und Kindern und Eltern so viel Zeit wie möglich in gewohnter häuslicher Umgebung zu ermöglichen.

„[...] und dass man halt vermeidet, versucht, dass es dem Kind so gut wie möglich zuhause geht und man verhindert halt vermehrte stationäre Aufenthalte." (s_001; S.4, Z. 108-110)

\subsubsection{Kommunikation mit der Familie}

Die Konfrontation mit der Diagnose einer lebenslimitierenden Erkrankung des eigenen Kindes bringt die betroffenen Familien in eine vollkommen neue Lebenssituation, die sie begreifen, akzeptieren und bewältigen müssen. Voraussetzung für das Ansprechen einer palliativen Versorgungsstruktur ist nach Meinung der stationär tätigen und niedergelassenen Pädiater die Akzeptanz der Erkrankung durch die Familien und die Bereitschaft, Unterstützung anzunehmen. Laut Aussage einiger der befragten Kinderärzte entwickelt sich im Laufe der (Berufs-)Zeit ein Gespür dafür, den richtigen Moment zu erkennen, um den Familien professionelle Hilfe anzubieten.

„[...] da muss man schauen, ich glaub das ist, man muss, irgendwann entwickelt man ein Gefühl dafür, wann das dran ist [...]“(s_008; S. 3, Z. 90-91)

Auch in diesem Kontext wurde von den befragten Pädiatern ein frühzeitiges Ansprechen postuliert, um die Lebensqualität des Patienten zu wahren und zu verbessern.

„[...] man sollte es nicht zu spät machen, ich glaube man kann halt den Eltern und vielleicht auch dem Kind oder ganz sicher dem Kind so einer Verzweiflung noch helfen und Schmerzen nehmen, die sie nicht ertragen müssen und man kann halt vielleicht noch ein bisschen schöne Zeit rausholen, wenn man das frühzeitig anfängt. Also ich würde, wenn ich den Fall hätte, würde ich versuchen das nicht zu spät zu machen." (n_007; S. 5, Z. 145-151)

Demgegenüber steht der Gedanke einiger der Kinderärzte, eine spezialisierte palliativmedizinische Versorgung dann anzusprechen, wenn eine Progredienz der Erkrankung auch für die Eltern erkennbar ist und sie in einer erneuten Krisensituation eine intensivmedizinische Therapie im Krankenhaus für ihre Kinder ablehnen. 
„[...] intensivmedizinische Maßnahmen bis hin zur Reanimation, [...] ich glaube, wenn man diese Situationen mit den Eltern ein oder zweimal durchsteht, dass die Eltern dann selbst [...] eher zu dem Punkt kommen, [...] wenn sie einmal das Sorgental durchschritten haben, [...] manchmal verstehen sie das auch besser, [...] wo man dann auf alle Fälle darüber nachdenkt." (s_009; S. 4- 5, Z. 143-163)

Betroffene und ihre Angehörigen erleben die Mitteilung einer infausten Prognose häufig als Grenzsituation und reagieren mit Gefühlen wie Angst, Kontrollverlust, Hilflosigkeit, Wut und Hoffnungslosigkeit. Sowohl die befragten stationär tätigen Pädiater, als auch die niedergelassenen Pädiater geben zu bedenken, dass das Anbieten einer palliativen Versorgungsstruktur Hoffnungslosigkeit verstärken und das Gefühl vermitteln kann, dass es keinerlei Perspektive mehr für das erkrankte Kind und die Familie gibt.

„Das ist ziemlich schwierig, weil da natürlich sozusagen man und die Eltern gleich das Gefühl haben, nach dem Motto, jetzt ist jede Hilfe zu spät, jetzt geht's nur noch darum, das Sterben zu humanisieren oder wie auch immer [...]." (s_008; S.3, Z. 85-88)

\subsection{Weitere Ergebnisse der Interviews unabhängig von der Forschungs- frage}

\subsubsection{Rolle der behandelnden Pädiater}

Viele der niedergelassenen Pädiater sehen ihren Zuständigkeitsbereich nicht in der Übermittlung einer lebenslimitierenden Diagnose. Häufig haben Kinderärzte in ihrer Praxis selbst keine eigenen Patienten, die lebenslimitierend erkrankt sind und wenig bis gar keine Berührungspunkte mit Patienten, für die eine palliative Mitversorgung in Frage kommt. In den Interviews wurde häufig berichtet, dass es kaum eigene Erfahrungen mit palliativ erkrankten Kindern im ambulanten Bereich gibt.

„Es ist schwierig, weil wir solche Patienten hier im Alltag nicht betreuen, deswegen habe ich keine Erfahrung damit. [...] also solche kritisch kranken Kinder werden ja meistens nicht nur durch die Niedergelassenen betreut, sondern eben hauptsächlich eigentlich an einer spezialisierten Ambulanz [...] und insofern werden solche Schritte oder solche Meilensteine sozusagen ja eigentlich eher von anderen beobachtet. Deswegen haben wir da oder ich habe keine eigene Erfahrung [...]. (n_010; S.2, Z. 42-50)

Die Diagnose einer unheilbaren Erkrankung wird nach Ansicht der befragten niedergelassenen Pädiater in aller Regel in der Klinik gestellt.

„[...] Ich bin eigentlich nie in der Situation, dass ich der Übermittler dieser Diagnosen bin 
[...] es ist meistens so, dass die entsprechenden Zentren die Diagnose gestellt haben, auch die Erstgespräche führen, mit der Frage, was bedeutet diese Diagnose für das Kind [...]." (n_002; S. 2-3, Z. 64-73)

In der Klinik erfolgt von den stationär tätigen Kollegen ein erstes Aufklärungsgespräch mit den Eltern und weitere Behandlungsstrategien werden diskutiert und festgelegt. Je nach klinischem Zustand des Patienten und dessen ambulanter oder stationärer Aktenlage entscheidet sich dann auch in der Klinik, ob ein Patient im Verlauf eine Palliativversorgung benötigt.

„[...] wir entscheiden uns hier vor Ort in der Kinderklinik anhand der stationären oder ambulanten Aktensituation und des klinischen Verlaufes, dass ein Patient Palliativversorgung benötigt."(s_010; S.1, Z. 10-13)

Wird bei einem Patienten in der Klinik von den behandelnden Ärzten die Indikation für eine palliativmedizinische Versorgung gestellt, so besteht dort bereits während des stationären Aufenthaltes jederzeit die Möglichkeit, Kollegen des palliativmedizinischen Versorgungsteams für weitere Entscheidungen und Gespräche zu Rate zu ziehen. Eltern und Kind können einzelne Personen des Palliativteams kennenlernen und gemeinsam die geplante Betreuung zuhause besprechen. Aus Sicht der stationär tätigen Pädiater herrscht eine gute Kommunikation und Zusammenarbeit zwischen stationär tätigen Pädiatern und Kollegen des Palliativteams, die als Ansprechpartner zur Verfügung stehen.

\subsubsection{Erfahrungen mit Palliativmedizin}

Insgesamt wurden die Erfahrungen der stationär tätigen und niedergelassenen Pädiater mit palliativen Versorgungsstrukturen als überwiegend positiv und gut beschrieben. Sowohl Eltern als auch Patienten waren aus Sicht der Pädiater mit der Betreuung im häuslichen Umfeld zufrieden und erfuhren Unterstützung, Hilfe und Entlastung. Auch die Mediziner sind froh, dass es diese Art der Unterstützung und die Möglichkeit palliativer Versorgung gibt.

„Also eigentlich hab ich sehr gute Erfahrungen. Ich bin froh, dass es das gibt, muss ich ehrlich sagen, [...] das ist ganz gut organisiert." (s_006; S.6; Z. 180-182)

Die spezialisierte ambulante pädiatrische Palliativversorgung schafft die Möglichkeit schwerstkranke Kinder Zuhause zu begleiten, Krankenhausaufenthalte zu vermeiden, ihre Lebensqualität aufrechtzuerhalten oder zu verbessern und ein Sterben in gewohnter Umgebung zu ermöglichen. So viel wie möglich Zuhause sein zu können und soweit 
möglich am alltäglichen Leben teilzunehmen, ist ein bedeutender Wunsch vieler Patienten mit einer lebenslimitierenden Erkrankung.

"[...] und wir haben es geschafft, seit wir involviert waren, dass die nicht mehr ins Krankenhaus kommen musste. das war schon gut, also das konnten wir alles ambulant managen bis dahin und da war auch klar, dass die Eltern jetzt sie gehen lassen wollten, die wollten auch nicht wieder noch mal in die Klinik." (s_008; S.3, Z. 73-75)

„[...] das Kind war palliativ versorgt und [...] ist dann im Rahmen der nächsten Krise zuhause verstorben und ich glaube es war für alle Beteiligten tatsächlich relativ zufriedenstellendes Ergebnis dann sozusagen der weiteren Betreuung gewesen." (s_009; S.3, Z. 104-108)

\subsubsection{Bedeutung von ambulanter pädiatrischer Versorgung aus Sicht der Pädiater}

Palliativmedizinische pädiatrische Betreuung bedeutet für die befragten Pädiater in erster Linie die ambulante pflegerische, medizinische und psychosoziale Versorgung von lebenslimitierend erkrankten Kindern und deren Familien in ihrem gewohnten Umfeld. Eine spirituelle Betreuung des Patienten und der Familie wurde nur von einem der befragten niedergelassenen Pädiater spontan genannt. Im Vordergrund der ambulanten Palliativversorgung steht u.a. die Vermeidung von stationären Aufenthalten, die Gestaltung eines möglichst normalen Alltages für die Kinder und die Erhaltung bzw. Verbesserung ihrer Lebensqualität durch Lindern von leidvollen Symptomen wie z. B. Krampfanfällen, Schmerzen, Unruhe, Schlafstörungen und die Entlastung und Unterstützung der gesamten Familie. Daneben ist die Akzeptanz einer Erkrankung und Akzeptanz dessen, dass ein Kind sterben darf, wichtiges Thema der palliativmedizinischen Versorgung. Kinder haben gleichermaßen wie Erwachsene das Recht in Würde und im Beisein ihrer Angehörigen zu sterben. Aufgabe des Palliativteams ist es, den Weg des Kindes und dessen Familie im Leben, im Sterben und über den Tod hinaus zu begleiten.

\subsubsection{Wünsche}

Die Idee, schwerstkranke und sterbende Menschen palliativmedizinisch zu begleiten und zu betreuen, hat ihren Ursprung bereits in den 1960er und 1970er Jahren. Trotzdem gilt die Palliativmedizin heute als junge und wachsende Disziplin. Auch die Befragung der stationär tätigen und niedergelassenen Pädiater hat gezeigt, dass es ein deutliches Interesse an Fort- und Weiterbildung zu diesem Thema gibt.

„Ich fände nicht schlecht, wenn diese, das ist ja was, was in den letzten Jahren glaube ich sehr forciert wird auch, diese Palliativversorgung, liest man zumindest. wenn's da 
vielleicht mal, vielleicht gab's das auch schon längst, oder wiederholt, regelmäßig Fortbildungsveranstaltungen für die Niedergelassenen gäbe [...].“ (n_010; S.5, Z. 122-127)

Zur Ausbildung und zum Beruf des Pädiaters gehört auch die Auseinandersetzung mit Sterben und Tod. Die Übermittlung einer lebenslimitierenden Diagnose und die Begleitung der Familie in einer Situation, die das gesamte Leben einer Familie plötzlich verändert, stellt gerade für junge Kollegen eine große Herausforderung und manchmal auch persönliche Belastung dar.

„Dann waren sozusagen die Assistenten halt auch zuständig und da hat mir einfach ne Ausbildung gefehlt. ich glaube es, es ging gut und die Eltern und die Eltern waren einfach dankbar, wenn man gekommen ist und mit ihnen geredet hat, aber ich fühlte mich da oft überfordert. einfach in der Gesprächsführung, in der Handhabung, [...] nicht das ich mir was vorwerfe, dass ich was falsch gemacht hab oder so, aber das war schon ne Situation, die sehr besonders ist und auch sehr, emotional lang anhaltend." (s_006; S. 5, Z. 134-143)

Nach Meinung der befragten Pädiater sollte die Palliativmedizin ebenso wie kurative Therapiemöglichkeiten zum Themenkatalog der Facharztausbildung gehören und fester Bestandteil der Ausbildung zum Pädiater sein. Kurative und palliative Medizin sollten ganz selbstverständlich miteinander verzahnt sein und nebeneinander praktiziert werden. Weiterhin sollten sowohl stationär tätige als auch niedergelassene Pädiater für Palliativmedizin sensibilisiert werden.

„[...] es gehört mit zur Ausbildung zum Kinderarzt dazu, sich über Palliativmedizin auch zu informieren und zu wissen, was das bedeutet. [...] wir als Kinderklinik [...] sind auch aufgefordert, darüber aufzuklären und auszubilden. Sie gehört zum Alltag in einer Kinderklinik dazu, insbesondere wenn man eine Intensivstation hat, auf der natürlich viele Kinder auch mit lebenslimitierenden Erkrankungen auch, ja versorgt werden können und auch der ambulant tätige Kinderarzt muss Strukturen kennen, in denen er palliativmedizinisch tätig werden kann." (s_010; S. 3, Z. 95-104)

Im Idealfall gibt es sowohl eine eigene Kinderpalliativstation oder „Palliativbetten“ in einer Kinderklinik, als auch ambulante Versorgungsstrukturen als Teil der Klinikstruktur. Auf diese Weise kann eine frühzeitige Einbindung einer ambulanten Versorgungsstruktur ermöglicht und der Übergang von der stationären zur ambulanten Behandlung erleichtert und bestmöglich koordiniert werden. 
Weitere Wünsche zielen auf die Möglichkeit einer adäquaten und flächendeckenden Versorgung für lebenslimitierend erkrankte Kinder und die nötige Zeit, die eine solche Aufgabe in Anspruch nimmt, ab. Im Moment ist die ambulante Palliativversorgung für den betreffenden Pädiater in vielen Fällen eine Zusatzaufgabe neben den alltäglichen Anforderungen in der Klinik oder der Praxis. Einige der befragten Pädiater bedauern, nicht mehr Zeit für die Versorgung von lebenslimitierend erkrankten Kindern und die Organisation dieser Aufgabe zu haben.

"[...] wir machen es halt on top und das ist halt ein bisschen unglücklich, dass man einfach 'n bisschen wenig Zeit hat dafür, dass man sich mehr Zeit wünschen würde." (s_008; S.6, Z. 193-195) 


\section{Diskussion}

Das Ziel dieser Arbeit bestand in der Erfassung von Argumenten für die Zuweisung von pädiatrischen Patienten zu einer palliativmedizinischen Mitbehandlung aus der Perspektive von niedergelassenen und stationär tätigen Pädiatern. Es sollten Zuweisungskriterien herausgearbeitet werden, die zu einer frühzeitigen kooperativen palliativmedizinischen Mitbetreuung von pädiatrischen Palliativpatienten führen. Eine Studie mit dieser Fragestellung ist nach dem Wissen der Doktorandin bisher nicht durchgeführt worden. Die Beschäftigung mit dem Thema ist neu und soll hier auf die Bedeutung einer frühzeitigen palliativmedizinischen Mitbetreuung von pädiatrischen Palliativpatienten hinweisen und das Bewusstsein für eine frühe Integration von Palliativmedizin in der Pädiatrie schärfen. Grundlage dieser Arbeit bildet das Interviewmaterial einer semistrukturierten Befragung niedergelassener und stationär tätiger Pädiater. Da sich die Fragestellung dieser Arbeit auf die Darstellung subjektiver individueller Ansichten und Einstellungen der befragten Pädiater bezog, bot sich eine überwiegend qualitative Herangehensweise als Forschungsdesign an. Durch ihren offenen Ansatz erlauben qualitative Methoden eine subjektorientierte Forschung (MAYRING 2002). Es können Wissenslücken geschlossen und neue Kenntnisse erschlossenen werden (KUCKARTZ et al. 2008). Durch die Anwendung qualitativer Methoden lässt sich ein komplexes Phänomen in seiner ganzen Breite und Tiefe erfassen (BROSIUS und KOSCHEL 2001). Zusätzlich ermöglichte in dieser Studie die getrennte Betrachtung der Aussagen der niedergelassenen und stationär tätigen Pädiater einen direkten Vergleich in der Auswertung der Interviews der befragten Personengruppen. Qualitative Studien zeichnen sich durch ein offenes Vorgehen und dem Subjekt im Vordergrund der Analyse aus (MISOCH 2015). Vor dem Hintergrund quantitativer Datenerhebungen scheint die Anzahl der Studienteilnehmer bei qualitativen Studien wie der vorliegenden zunächst gering. Im Gegensatz zu quantitativen Studien, die ihre Aussagekraft über große Stichproben und bestimmte Merkmalszusammenhänge oder Ausprägungen nach dem Gesetz der „großen Zahl“ erreichen (MISOCH 2015), streben qualitative Studien durch die Ermittlung individueller Sichtweisen und dem Verstehen des Subjekts „von innen heraus“ danach, die Sicht des Befragten nachzuvollziehen. Der qualitative Ansatz dieser Studie ermöglichte es, die subjektive Sicht der befragten Personen zu erfassen, ein differenziertes Bild an Aussagen zu erheben und somit bisher unbekannte Sachverhalte zu entdecken, die mit einer quantitativen Herangehensweise weniger gut oder überhaupt nicht zu erfassen gewesen und im Verborgenen geblieben wären.

In die Auswertung dieser Studie wurde eine Gruppe von zehn niedergelassenen und 
zehn stationär tätigen Pädiatern eingeschlossen. Der initial von den befragten Personen bearbeitete Fragebogen der Untersuchung, diente der Datenerhebung. Er ermöglichte es, zählbare Eigenschaften wie personenbezogene Angaben der Pädiater, ihrer Patienten und ihr Verständnis der Verfügbarkeit von spezialisierter Palliativversorgung in der Umgebung, abzubilden. Unproblematisch bei der Bearbeitung des Fragebogens waren Themen, die durch ankreuzen einer oder mehrerer zutreffender Variablen beantwortet werden konnten. Schwierigkeiten bei der Beantwortung ergaben sich insbesondere bei der geforderten Angabe von geschätzten Durchschnittswerten (z.B. geschätzte Häufigkeit eines palliativen Behandlungsansatzes). Vor allem wurden einige der offenen Fragen zum Gegenstand der persönlichen Beschäftigung mit palliativmedizinischen Aspekten sowohl von niedergelassenen, als auch von stationär tätigen Pädiatern nicht beantwortet. Insgesamt gab es aus Sicht der Befragten keine wesentlichen Probleme mit den Anforderungen des Fragebogens. Beiden Gruppen der Befragten sahen als Voraussetzung für die Inanspruchnahme palliativmedizinischer Versorgungsstrukturen zunächst die Diagnose einer lebenslimitierenden Erkrankung. Eine Vielzahl der teilnehmenden Pädiater wünschte sich im Rahmen einer lebenslimitierenden Erkrankung ein möglichst frühes Angebot palliativmedizinischer Versorgungsstrukturen. Ein konkreter Zeitpunkt der Inanspruchnahme wurde nicht benannt. Der individuell unterschiedliche Verlauf einer Erkrankung und die Akzeptanz einer lebenslimitierenden Erkrankung durch den Patienten und die Familie, fließt laut Aussage der Befragten in die Entscheidung für eine palliativmedizinische Versorgung mit ein. Es ist ein Entwicklungsprozess, der individuell sehr unterschiedlich verläuft. Unterschiede zeigten sich in den Aussagen zur eigenen Rolle von stationär tätigen und niedergelassenen Pädiatern. Im Folgenden werden die Ergebnisse der Studie genauer betrachtet.

\subsection{Stärken und Schwächen der Studie}

Kriterien der Qualitätssicherung sind sowohl bei quantitativer als auch bei qualitativer Forschung von zentraler Bedeutung. In der hier vorliegenden Studie wurde zur wissenschaftlichen Erarbeitung der Fragestellung ein überwiegend qualitatives Forschungsdesign gewählt. Um die Qualität qualitativer Forschungsansätze sicherzustellen, ist zunächst die bewusste und begründete Wahl der qualitativen Methoden ein Gütekriterium qualitativer Arbeiten. Es ist auf die Indikation qualitativer Forschung zu achten (FLICK 2002). Generell eignet sich die Qualitative Inhaltsanalyse zur Analyse von Problemen, Kulturen und Sachverhalten (MAYRING 2000) (LAMNEK 2005) besonders dann, wenn die Absicht des Untersuchers eine Reduktion und Zusammenfassung des Ausgangsmateriales ist. Da bei dieser Arbeit die Erfassung von persönlichen Sichtweisen, Erfahrungen und Erlebnissen der befragten Pädiater und ihre konkreten und detaillierten Antworten im Vordergrund standen und größere Textmengen ausgewertet werden mussten, 
war eine inhaltsanalytische Vorgehensweise sinnvoll. Die drei klassischen Gütekriterien der Qualitätssicherung quantitativer Forschung lassen sich nicht ausdrücklich und ohne hinterfragt zu werden auf die qualitative Forschung übertragen. Aufgrund der Systematik des Vorgehens und der Regelgeleitetheit erhebt qualitative Inhaltsanalyse den Anspruch, prinzipiell nachvollziehbar zu sein. Transparenz und Intersubjektivität sind damit Bestandteile des Ansatzes (MAYRING 2007). Allerdings birgt das Ablaufmodell nach Mayring die Gefahr, dass durch vorschnell gebildete Kategorien inhaltliche Feinheiten verloren gehen. Um dem entgegenzuwirken wurde die Paraphrasierung und Kategorienbildung aus dem Text heraus regelmäßig im Team der „Forschungswerkstatt“ diskutiert, überprüft und reflektiert. So konnte sichergestellt werden, dass das Material aus unterschiedlichen Perspektiven betrachtet wurde. Das ständige Feedback der Gruppenteilnehmer steigerte die Qualität der Arbeit (PRESS 2005). Die unabhängige Betrachtung und Bearbeitung des Textmaterials verschiedener Wissenschaftler zu bestimmten Textabschnitten wird von Mayring als Intercoderreliabilität bezeichnet und zu den Gütekriterien der qualitativen Inhaltsanalyse gezählt (MAYRING 2007). Bei der Durchführung qualitativer Interviews ist die Qualität der Daten zwangsläufig von der Qualifikation des Interviewenden abhängig und bestimmt nach Lamnek (LAMNEK 2005) die Tiefe der Interpretation, die anhand des Kategoriensystems vorgenommen werden. Neben der wissenschaftlichen Vorbereitung auf das Forschungsthema und der Aneignung konkreter Kommunikationstechniken muss der Interviewende laut Hopf (HOPF 1987) über kommunikative Kompetenzen und eine gewisse Eloquenz verfügen sowie die Fähigkeit besitzen, Ruhe, Wärme und Offenheit auszustrahlen (MISOCH 2015). Eine Interviewschulung mit der Sozialwissenschaftlerin der Forschungsgruppe und die Durchführung und Reflexion eines Probeinterviews im Vorfeld der Studie bot die Möglichkeit, persönliche Unsicherheiten mit Kommunikationstechniken abzulegen und Selbstvertrauen in der Gesprächsführung aufzubauen. Kritiker qualitativer Forschungsmethoden beanstanden insbesondere die Subjektivität der Datenerhebung und werfen ihr Unwissenschaftlichkeit vor. In qualitativen Studien liegt jedoch die zentrale Aufgabe des Interviewenden darin, eine intensive Beziehung zum Befragten herzustellen (ADLER und ADLER 2002; KVALE 1996) und dadurch eine Interviewatmosphäre zu ermöglichen, in der aussagekräftige und authentische Daten erhoben werden können. Wichtig ist hierbei der reflektierte und kontrollierte Umgang mit der Rolle von Subjektivität und des Einflusses des Interviewenden innerhalb des qualitativen Forschungsprozesses (MISOCH 2015). Die qualitative Erhebung und Analyse von Daten ist immer ein kommunikativer und interaktiver Prozess zwischen dem Forscher und den Untersuchten (WOLF 2008). Mit der Auswahl eines qualitativen Ansatzes gelang es in dieser Studie umfassende und differenzierte Aussagen zu erheben. Mit einem quantitativen Forschungsansatz wären wesent- 
liche Aspekte sicherlich nicht zur Sprache gekommen und somit wichtige Ansichten verloren gegangen. Neben der qualitativen Analyse des Textmateriales der Interviews fand im Vorfeld der Interviews eine Befragung der Studienteilnehmer mittels Fragebogen statt. Die Aussagen des Fragebogens dienten dazu unterschiedliche persönliche Daten der befragten Pädiater, Angaben zu ihren Patienten und die Verfügbarkeit spezialisierter palliativer Versorgungsstrukturen zu erfassen, welche nicht den Anspruch haben, repräsentativ zu sein, für die Fragestellung und Diskussion der Ergebnisse jedoch sehr hilfreich waren und somit später in die Auswertung mit einflossen. Laut Mayring erhält man durch die Kombination unterschiedlicher Forschungsmethoden die größte Chance, die jeweiligen Vorteile der beiden Forschungstraditionen zu nutzen (MAYRING 2001). Mit dem Forschungsansatz dieser Arbeit ist es weniger gelungen, die frühe Indikationsstellung für eine palliativmedizinische Mitversorgung, also den Zeitpunkt, sondern mehr die Kriterien für eine Inanspruchnahme palliativer Versorgungsstrukturen herauszuarbeiten. Durch bewusste Auswahl der Studienteilnehmer, bei denen die Voraussetzung für die Teilnahme an der Studie die Facharztbezeichnung für Pädiatrie darstellte, wurde eine Studienpopulation untersucht, von der spezifisches Wissen und Interesse an der Forschungsfrage erwartet wurde. Die Studienteilnehmer konnten mit ihrer Kompetenz, ihrem Fachwissen und ihren persönlichen Erfahrungen dazu beitragen, der Fragestellung des bestehenden Forschungsbedarfes nachzukommen und erlaubten eine Annäherung an die Forschungsfrage. Diese gezielte Auswahl ging aber durchaus mit einer Selektion der Studienpopulation einher. Pädiater, von denen keine Interviewbereitschaft bzw. kein Interesse am Thema Palliativmedizin erwartet wurde, standen nicht im Blickfeld der Befragung. Ein weiterer Aspekt bei der Durchführung dieser Studie war, dass ein großer Teil der Studienteilnehmer persönlich mit der Interviewerin bekannt war. Diese Tatsache kann zu einer grundsätzlich höheren Teilnahmebereitschaft an der Studie und einem größeren Engagement der Studienteilnehmer geführt haben, bildet aber möglicherweise nicht das tatsächliche Interesse der Pädiater an der Forschungsfrage ab. Auch ist eine besonders positive oder vorteilhafte Beantwortung der gestellten Fragen denkbar. Ein Nachteil der qualitativen Inhaltsanalyse nach Mayring ist sicherlich darin zu sehen, dass bei der Auswertung der Interviews sämtliche Informationen, die keine Antwort auf die Forschungsfrage geben verloren gehen und möglichen weiteren interessanten Aspekten hierbei keine Bedeutung zukommen.

\subsection{Studienpopulation}

Um die Forschungsfrage möglichst intensiv und effektiv bearbeiten zu können, wurden die Interviewpartner so ausgewählt, dass eine generelle Interviewbereitschaft der Be- 
fragten mit hoher Wahrscheinlichkeit vorausgesetzt und differenzierte forschungsrelevante Aussagen der Betreffenden erwartet werden konnten. In der qualitativen Forschung rückt die Strategie der bewussten Fallauswahl in das Zentrum der Untersuchung. Diejenigen Subjekte werden in die Arbeit einbezogen, die sich als inhaltlich adäquat im Hinblick auf die Forschungsfrage erweisen und die inhaltlich reichhaltige Informationen versprechen (MISOCH 2015). Die Auswahl der Befragten erfolgt nicht nach Repräsentativitätskriterien, daher werden keine Zufallsstichproben gezogen. Es interessieren vielmehr typische Fälle (LAMNEK 2005). Eine derartige Auswahl der Studienpopulation birgt die mögliche Gefahr der fehlenden Berücksichtigung aller relevanten Inhalte, zumal die Auswahl der Interviewpartner häufig auch von forschungspraktischen Kriterien wie zum Beispiel der Interviewbereitschaft abhängig ist. Außerdem steht sie auch dem Anspruch qualitativer Forschung konträr gegenüber, individuelle Sichtweisen von innen heraus zu ermitteln und die Auswahl nicht nach äußeren Attributen zu treffen. Auch wenn die Analyse das Fehlen wesentlicher Aspekte offenbart, ist es andererseits bei dieser Methode auch möglich, weitere Daten zu einem späteren Zeitpunkt zu erheben. In der vorliegenden Arbeit ist die hundert prozentige Teilnahme und Mitwirkung der angefragten Pädiater an der Studie als hoch zu bewerten. Unter den Befragten zeugt dies von einer hohen Bereitschaft der niedergelassenen und stationär tätigen Pädiater, sich mit dem Thema auseinanderzusetzen und lässt ein auffallendes Bewusstsein für die Bedeutung der Palliativmedizin in der Pädiatrie erkennen. Sowohl in der Studienpopulation der zehn niedergelassenen Pädiater als auch der Gruppe der stationär tätigen Pädiater war das Verhältnis von weiblichen und männlichen Interviewpartnern nahezu ausgeglichen. Ein Grund für die Ausgeglichenheit der Geschlechterverteilung ist möglicherweise in der allgemeinen geschlechtsneutralen Aufgeschlossenheit der Berufsgruppe der Pädiater gegenüber dem Forschungsthema zu sehen, aber auch mögliches Ergebnis der gezielten Anfrage der betreffenden Personen bezüglich der Bereitschaft der Teilnahme an der Befragung im Rahmen dieses Projektes. Das Durchschnittsalter lag bei den stationär tätigen Pädiatern sieben Jahre unter dem der niedergelassenen Pädiater und auch die Approbation hatten die Teilnehmer aus der Gruppe der stationär tätigen Pädiater durchschnittlich sieben Jahre früher erhalten als die Befragten der Gruppe der niedergelassenen Pädiater. Der Unterschied im Altersdurchschnitt und die größere Summe an Jahren nach Erhalt der Approbation lassen sich damit erklären, dass eine Niederlassung erst nach einer gewissen Zeit der stationären Tätigkeit im Krankenhaus und nach absolvierter Facharztprüfung möglich ist. Die meisten Pädiater beenden ihre stationäre Tätigkeit und gehen den Schritt in die Niederlassung erst nach qualifizierter Weiterbildung und gewonnener klinischer Erfahrung, so dass das Alter bei Eintritt in die Niederlassung meist per se höher ist als bei Weiterführung der stationären Tätigkeit nach Erhalt der Facharztbezeichnung. Der Altersdurchschnitt von 41 Jahren bei den stationär tätigen 
und 48 Jahren bei den niedergelassenen Pädiatern und eine Dauer der ärztlichen Tätigkeit mit durchschnittlich 15,5 Jahren und 22 Jahren belegen die Teilnahme überwiegend erfahrener Pädiater an den Interviews. Die Zusatzbezeichnung „Palliativmedizin“ hatte unter den niedergelassenen Pädiater niemand erlangt, zwei der stationär tätigen Pädiater hatten zum Zeitpunkt der Befragung an der Weiterbildung „Palliativversorgung bei Kindern- und Jugendlichen“ mit dem Erwerb der Zusatzbezeichnung „Palliativmedizin“ teilgenommen. Parallelen zwischen der Dauer der beruflichen Tätigkeit und qualifizierter, kompetenter Erfahrung in der Betreuung lebenslimitierend erkrankter Kinder können daher kaum gezogen werden. Es drängt sich also die Frage auf, warum trotz des gesteigerten Interesses und der Bedeutung des Themas Palliativmedizin in der Pädiatrie für die Befragten, wenig Ärzte dabei waren, die sich weiter in der Palliativmedizin spezialisiert haben. Einer der Gründe könnte die hohe Arbeitsbelastung der Mediziner sein (KNESEBECK et al. 2010). Die Gesamtsituation habe sich zwar laut Studienergebnissen gebessert, allerdings seien noch immer eine Wochenarbeitszeit von rund 60 Stunden die Regel in der Chirurgie, Pädiatrie und Gynäkologie (SCHIKORA 2007). Als Ursache für das Vorliegen der Zusatzbezeichnung „Palliativmedizin“ ausschließlich in der Gruppe der stationär tätigen Pädiater ist nach Aussagen der Befragten in dieser Arbeit die Tatsache, dass lebenslimitierend erkrankte Patienten überwiegend in spezialisierten Zentren und nicht im ambulanten Bereich betreut werden. Berührungspunkte mit Patienten, die eine palliative Mitversorgung benötigen, seien in der Praxis selten (siehe 6.6.4.). Insgesamt ist die Zahl der Mediziner mit einer Zusatzausbildung für Palliativmedizin (für den Erwachsenen- sowie für den Kinder- und Jugendbereich) von ca. 100 Ärzten im Jahr 2005 auf etwa 8000 Ärzte im Jahr 2013 gestiegen (GESUNDHEITSBERICHTERSTATTUNG des BUNDES 2015). Im Zeitraum der Befragung hatten zwei der befragten stationär tätigen Studienteilnehmer die Zusatzbezeichnung „Palliativmedizin“ erlangt. Inzwischen sind vier der befragten stationär tätigen Pädiater „Palliativmediziner“. Grundsätzlich wächst mit der steigenden Zahl an komplex und chronisch erkrankten Menschen mit lebenslimitierenden Erkrankungen, die aufgrund des Fortschritts der Medizin und der pflegerischen Versorgungsmöglichkeiten (z.B. Intensivpflegedienste auch im ambulanten Bereich) auch zu Hause oder in Pflegeeinrichtungen (Pflegeheime und Hospize) versorgt werden können, der Bedarf an qualitätsgesicherter palliativmedizinischer Versorgung. Zunehmend werden mit dem Auf- und Ausbau der Palliativmedizin für Kinder, Jugendliche und junge Erwachsene spezialisierte Fachkräfte gebraucht und das Interesse an der noch recht jungen Disziplin nimmt zu. 


\subsection{Patientengruppe}

In der Auswertung der Fragebögen, die nicht repräsentativ ist, gaben die stationär tätigen Pädiater in ihrem Tätigkeitsfeld ein Patientenalter von der Geburt bis ins mittlere Erwachsenenalter an, womit die Betreuung der Patienten verschiedener Altersstufen, der Umgang mit den unterschiedlichen Bedürfnissen der jeweiligen Personen und einem äußerst breiten Spektrum an speziell im Kindes- und Jugendalter auftretenden Erkrankungen einhergeht. Diese Besonderheit in der Pädiatrie findet auch im § 37b SGB V (Spezialisierte ambulante Palliativversorgung) Beachtung, indem dort darauf hingewiesen wird, die besonderen Belange von Kindern mit einer lebenslimitierenden Diagnose zu berücksichtigen (HOSPIZ- und PALLIATIVGESETZ 2015). Der Altersdurchschnitt der Patienten im ambulanten Bereich liegt nach Aussage der hier befragten niedergelassenen Pädiater bei etwa vier bis fünf Jahren. Im ambulanten Bereich werden laut Aussage der Pädiater also vorwiegend Patienten des Kleinkind- bzw. Vorschulalters betreut. Es gibt Hinweise darauf, dass die ambulante Inanspruchnahme von Kinderärzten mit zunehmendem Alter der Patienten sinkt und zugleich die Rate der zu behandelnden Kinder in der Allgemeinmedizin ansteigt (RATTAY et al. 2014). Krankheitsbilder, mit denen sich die Gruppe der stationär tätigen Pädiater im klinischen Alltag auseinandersetzt, sind häufiger spezielle und zum Teil seltene Erkrankungen aus den zahlreichen Disziplinen der Pädiatrie. In der ambulanten Betreuung von pädiatrischen Patienten stehen die Behandlung von unterschiedlichsten Infektionserkrankungen und die Prävention von Krankheiten im Vordergrund. Hier lässt sich ein deutlicher Unterschied zwischen stationär tätigen Pädiatern und niedergelassenen Pädiatern bezüglich der Häufigkeit der zu behandelnden Grunderkrankungen im jeweiligen Arbeitsumfeld erkennen. Dieser Unterschied liegt am ehesten darin begründet, dass bestimmte Erkrankungen nicht ausschließlich ambulant behandelbar sind und diese Patienten zur weiteren Diagnostik und Therapie in die Klinik überwiesen werden. Ein palliativer Behandlungsansatz besteht nach Angaben der stationär tätigen Pädiater durchschnittlich bei annäherungsweise zwölf Prozent der im Krankenhaus behandelten Patienten. Die Diagnose einer lebenslimitierenden Erkrankung wird hier häufig im Bereich neurologischer, onkologischer und kardiologischer Erkrankungen gestellt. Die Zahl der Patienten mit palliativem Behandlungsansatz im ambulanten Bereich wurde von den niedergelassenen Pädiatern mit durchschnittlich 0,3 Prozent deutlich geringer eingeschätzt. Auch hier stehen neurologische und onkologische Krankheitsbilder im Vordergrund. Allerdings gibt es in der vorliegenden Untersuchung niedergelassene Pädiater, die in ihrem Praxisalltag bislang noch nie einen Patienten mit palliativem Behandlungsansatz behandelt haben. 


\subsection{Interviews}

Qualitative Forschung kann in Form unterschiedlichster Methoden geschehen. Allen Ansätzen gemein ist die nahe und intensive Beschreibung eines Phänomens. Die Qualitative Forschung definiert solche Phänomene als einen Forschungsgegenstand, die nicht auf einzeln messbare Variablen reduziert werden können. Die Forschungsgegenstände sind zumeist Konstrukte, die sprachlich eng an die Alltagswelt angelehnt sind (FUHS 2007). In Bezug auf das Interview war bei einigen der Befragten anfänglich eine gewisse Scheu spürbar. Möglich ist die Befürchtung der Interviewpartner, sie könnten im Gespräch nicht die „richtigen“ Antworten geben. „Richtige“ oder „falsche“ Antworten gibt es nicht, sondern nur jene, die verzerrt werden durch die Annahme des Interviewten, dass bestimmte Antworten erwartet würden (soziale Erwünschtheit) (MISOCH 2015). Aufgabe des Interviewers ist in dieser Situation, den Studienteilnehmern die Sorge vor „falschen" Antworten zu nehmen und eine angenehme und vertrauensvolle Situation zu schaffen, in der sich der Befragte frei und ohne den Druck sozialer Erwartungen äußern kann (MISOCH 2015). In der Interviewsituation gelang dies u.a. durch die Befragung der Pädiater in ihrem gewohnten Umfeld, welches innen Sicherheit vermittelte. Bevor ein Interviewteilnehmer frei spricht und einem Fremden seine persönlichen Erfahrungen und Gefühle offenbart, möchte er den Interviewer einschätzen können (KVALE 2007).

\subsection{Analyse des Materials}

Aufgrund der zielgerichteten Fragestellung dieser Arbeit war es von Bedeutung, konkrete und detaillierte Antworten zu generieren. Aus diesem Grund wurde für die Auswertung der Interviews die induktive Vorgehensweise der Kategorienbildung nach Mayring gewählt, da im Rahmen qualitativ orientierter Ansätze die Auswertungsaspekte nahe am Material, aus dem Material heraus entwickelt werden. Die Bildung der Kategorien erfolgt in dieser Studie durch Paraphrasierung, Generalisierung und Reduktion relevanter Textstellen. In einem nächsten Schritt werden die entwickelten Kategorien überprüft, gegebenenfalls überarbeitet und zusammengefasst. Auch das fertige Kategoriensystem erfährt in einem endgültigen Materialdurchgang erneute Testung. Kategorien werden damit immer aus dem Material entwickelt, überarbeitet, angepasst und rücküberprüft (MAYRING 2000). Diese Vorgehensweise verlangt bei der Projektausführung ein hohes Maß an Geduld und Ausdauer. Eine der Gefahren bei der Kodierung des Ausgangsmaterials besteht darin, zu schnell ein System der Kategorien zu erstellen und den Kontakt zu der Bedeutung, die im Text enthalten ist, zu verlieren (FLICK 2002; LAMNEK 2005). Aus diesem Grund wurden in der vorliegenden Arbeit einzelne transkribierte Textbeispiele und Codierungen des Materials regelmäßig dem Projektteam vorgestellt und im Rahmen einer stattfindenden „Forschungswerkstatt“ gemeinsam diskutiert und ggf. überarbeitet. 
Eine nur einseitige Betrachtung des Materials konnte so vermieden und das Einbeziehen möglichst vieler für das Kodierungssystem relevanter Aussagen gewährleistet werden. Eine Herausforderung während des Analyseprozesses war es, den Überblick über das Kategoriensystem zu behalten. Die Nutzung eines Computerprogrammes wie MAXQDA stellt bei der Neuzuordnung oder Umstrukturierung des Kategoriensystems eine deutliche Erleichterung dar. Eine händische Analyseermöglicht jedoch einen intensiveren Kontakt mit dem Material. Beide Möglichkeiten der Datenanalyse wurden in der vorliegenden Untersuchung verwendet. Die Analyse der Daten ohne den Einsatz von Computersoftware konnte bei der Bearbeitung des Textes den Zeitaufwand reduzieren und erlaubte eine äußerst präzise Auswertung des Materials.

\subsection{Verfügbarkeit palliativer pädiatrischer Versorgungsstrukturen in Göt- tingen - Engagement der Pädiater}

In der Stadt Göttingen mit Existenz der Universitätsmedizin, der zugehörigen Klinik für Kinder- und Jugendmedizin und dem Palliativzentrum Göttingen besteht eine qualifizierte und fachgerechte palliativmedizinische Behandlung und Betreuung von lebenslimitierend erkrankten Kindern und Jugendlichen. Sowohl die befragten niedergelassenen als auch die stationär tätigen Pädiater nennen unterschiedliche palliative Versorgungsstrukturen, wie die spezialisierte ambulante Palliativversorgung, den pädiatrischen Palliativpflegedienst, die ambulante Hospizbewegung und die pädiatrische Palliativstation (es stand zum Zeitpunkt der Befragung ein Palliativpflegezimmer zur Verfügung) der Kinderklinik als mögliche Betreuungseinrichtungen für Kinder und Jugendliche mit lebenslimitierenden Erkrankungen im Raum Göttingen. Hierbei werden die real zur Verfügung stehenden Strukturen meist richtig benannt. Sowohl im niedergelassenen Bereich, als auch bei den stationär tätigen Pädiatern ist ein Wissen über bestehende palliative Versorgungsstrukturen in Göttingen vorhanden. Im Zeitraum der Befragung gehören sechs aller stationär tätigen Pädiater zum Kinderpalliativteam Göttingen, von den niedergelassenen Pädiatern engagiert sich niemand zusätzlich in der spezialisierten ambulanten Versorgung von lebenslimitierend erkrankten Kindern. Ein Grund hierfür könnte der zusätzliche Zeitaufwand einer spezialisierten ambulanten Versorgung sein, aber auch die von den niedergelassenen Pädiatern geschilderte Tatsache, dass sie selber in ihrer Praxis kaum lebenslimitierend erkrankte Patienten betreuen. Im stationären Bereich beschäftigen sich die Pädiater in der Woche im Durchschnitt etwa anderthalb Stunden länger mit palliativmedizinischen Aspekten als die niedergelassenen Kollegen und würden sich auch mehr Zeit für palliativmedizinische Aspekte wünschen als die Pädiater in der Praxis. Sicherlich ist die Präsenz der Universitätsmedizin mit den genannten Versorgungsmöglichkeiten und die dortige Behandlung von lebenslimitierend erkrankten 
Kindern als möglicher Grund dafür zu sehen, dass die stationär tätigen Pädiater mehr Zeit für palliativmedizinische Aufgaben beanspruchen. Bezogen auf die Gesamtkinderzahl in Deutschland (etwa 15000000 Kinder) leiden Kinder eher seltener (etwa 22000 Kinder) an einer lebenslimitierenden Erkrankung (STATISTISCHES BUNDESAMT 2015) und der einzelne Pädiater ohne die Zusatzbezeichnung Palliativmedizin bzw. einer Beschäftigung außerhalb eines ausgewiesenen Zentrums für Kinder- und Jugendmedizin kommt eher selten mit lebenslimitierend erkrankten Kindern in Kontakt. Einhergehend mit einer geringen Dichte pro Versorgungsfläche gibt es eine geringe Anzahl an Patienten (ZERNIKOW und NAUCK 2008). Kinder mit einer lebenslimitierenden Erkrankung werden vermutlich bereits primär im Krankenhaus behandelt und nicht in der niedergelassenen Praxis. Zwei der befragten niedergelassenen Pädiater haben in ihrer Praxis noch nie einen Patienten mit einer lebenslimitierenden Erkrankung betreut. Jedoch sind sowohl etwa die Hälfte der befragten niedergelassenen als auch der stationär tätigen Pädiater daran interessiert, sich zusätzlich mit palliativmedizinischen Aspekten zu befassen. Eine qualitative Studie von NEILSON et al. (2015) aus Großbritannien setzt sich mit der Rolle des Hausarztes in der kinderonkologischen Palliativpflege auseinander. Es wurden semistrukturierte Interviews mit achtzehn Hausärzten und elf betroffenen Eltern nach dem Tod ihres Kindes geführt, die den Zeitdruck der Hausärzte und auch die persönliche Herausforderung, die die Arbeit mit sterbenden Kindern und ihren Familien für den niedergelassenen Kollegen bedeutet, benennen. Eine verbesserte Kommunikation und Zusammenarbeit von Hausärzten mit den entsprechenden Zentren könnte laut dieser Studie zu einer Zeitersparnis der Hausärzte und der Überwindung von Schwierigkeiten und Barrieren führen. Möglicherweise würde eine noch engere Zusammenarbeit von Stationär tätigen Pädiatern bzw. dem ambulanten spezialisierten Palliativteam und den niedergelassenen Pädiatern in Göttingen zu einem größeren Engagement der niedergelassenen Pädiater im Bereich der Palliativversorgung und zu einem Abbau von eventuell vorhandenen Unsicherheiten im Umgang mit lebenslimitierend erkrankten Kindern und ihren Familien führen.

\subsection{Kriterien der Inanspruchnahme einer spezialisierten ambulanten pädi- atrischen palliativmedizinischen Versorgungsstruktur}

Seit 2007 haben Kinder mit einer lebenslimitierenden Erkrankung in Deutschland gesetzlichen Anspruch auf eine spezialisierte ambulante pädiatrische Palliativversorgung. Die spezialisierte ambulante Palliativversorgung beabsichtigt ein höchstes Maß an Lebensqualität für den Patienten unter Berücksichtigung seiner individuellen Bedürfnisse und der umfassenden Unterstützung der gesamten Familie (PALLIATIVVERSORGUNGSRICHTLINIE 2007). Leidet ein Kind an einer lebenslimitierenden Erkrankung, so 
sind sich in der vorliegenden Studie niedergelassene und stationär tätige Pädiater einig, sollte die Inanspruchnahme palliativmedizinischer Versorgungsstrukturen und eine spezialisierte ambulante palliativmedizinische Mitbetreuung dieser Patienten erwogen werden. Wesentliche Voraussetzung für die Verordnung einer professionellen Palliativversorgung sind aus Sicht der Befragten hierbei der Wunsch des Patienten und die Bereitschaft der Eltern. Die unabdingbare Wichtigkeit dieser Bereitschaft und in erster Linie die Akzeptanz der Erkrankung durch die Eltern wurde als Bedingung für das Hinzuziehen palliativmedizinischer Versorgungsstrukturen vielfach betont. Der Weg der Auseinandersetzung mit der Erkrankung des Kindes und ihren Folgen beginnt für die Familien mit dem Zeitpunkt der Diagnosestellung und stellt eine vielgestaltige Entwicklung dar, die individuell sehr unterschiedlich verlaufen kann. Von den Befragten wurde geschildert, dass häufig mehrere Gespräche zwischen Eltern und behandelnden Ärzten stattfinden, bevor es für die Eltern möglich ist, sich der frühen Endlichkeit des Lebens ihrer Kinder bei Vorliegen einer lebenslimitierenden Erkrankung zu stellen und eine Unterstützung durch eine spezialisierte palliativmedizinische Versorgungsstruktur zuzulassen. Sicherlich ist dies neben einer ersten natürlichen Reaktion des nicht-wahr-haben-Wollens (KÜBLER-ROSS 2014) auch auf die bei Kindern äußerst verschiedenartigen Diagnosen in unterschiedlichen psychosozialen und kognitiven Entwicklungsstadien und damit verbundenen individuell geprägten komplexen Symptomgeschehen zurückzuführen (ZERNIKOW und NAUCK 2008). Im Unterschied zur Betreuung schwerstkranker erwachsener Patienten ist vor allem bei Kindern mit chronischen Erkrankungen, wie z. B. der Spinalen Muskelatrophie, zum Zeitpunkt der Diagnosestellung das Lebensende längst nicht absehbar. Lebenslimitierende Erkrankungen bei Kindern bedürfen zum Teil über mehrere Jahre einer professionellen Begleitung und Unterstützung der Patienten und ihrer Familien. Leidvolle Symptome können zwischenzeitlich abklingen oder auch vorübergehend gar nicht mehr vorhanden sein, so dass eine spezialisierte ambulante Palliativversorgung pausiert werden kann. Diese besondere Situation trägt sicherlich dazu bei, dass es für die Eltern schwierig ist, sich die Präsenz der Endlichkeit des Lebens ihres Kindes vor Augen zu führen und sich für eine palliative Mitversorgung zu entscheiden. Dennoch profitieren betroffene Kinder von einer frühen Integration von Palliativmedizin, indem spezialisierte Versorgungsteams von Anfang an individuelle Bedürfnisse und Wünsche des Kindes und der Familie berücksichtigen und im Verlauf der Erkrankung Entscheidungen leichter getroffen werden können (MACK und WOLFE 2006). Ein weiteres wesentliches Merkmal für die Inanspruchnahme palliativmedizinischer Versorgungsstrukturen stellt darüber hinaus sowohl bei niedergelassenen als auch bei stationär tätigen Pädiatern eine erkennbare Notwendigkeit professioneller psychosozialer, pflegerischer und medizinischer Unterstützung für den Patienten und die Familie dar. An dieser Stelle soll angesprochen sein, dass in den durchgeführten Interviews für diese 
Studie mit den Ärzten die geistliche, spirituelle Begleitung der Patienten und ihrer Familien nur ein einziges Mal Erwähnung fand. Einer Umfrage von Hans Georg Ziebertz zufolge liegt die persönliche Bedeutsamkeit von Religion heute merklich unter fünfzig Prozent (ZIEBERTZ 2016). Möglich ist, dass Spiritualität bzw. Religion als ein Ausdruck von Spiritualität für die befragten Kinder- und Jugendmediziner kein zentrales Thema darstellt, möglicherweise aber Patienten und ihre Familien durchaus beschäftigt. Diese Frage kann hier jedoch nicht hinreichend beantwortet werden. Sind Eltern mit der Gesamtsituation zuhause überfordert und benötigen Unterstützung in den genannten Bereichen, kommt eine palliativmedizinische Mitversorgung in Betracht, wenn alternative ambulante Versorgungsmöglichkeiten ausgeschöpft sind. Hierbei ist es in der Regel nicht so, dass betroffene Familien die spezialisierte ambulante pädiatrische Palliativversorgung für sich einfordern, sondern es kommt nach Ansicht der befragten Kinderärzte auf eine achtsame Beobachtungsgabe und sensible Einschätzung des behandelnden Pädiaters an, die Dringlichkeit einer palliativmedizinischen Versorgung zu erspüren. Einzelne der niedergelassenen Pädiater führten auch das Erreichen eigener Grenzen der Kompetenz in der Betreuung lebenslimitierend erkrankter Kinder und Jugendlicher als ein Entscheidungskriterium für das Hinzuziehen eines professionellen Palliativteams an. Auch hier gilt es, professionelle Hilfe zu beanspruchen, um dem Ziel der Verbesserung von Lebensqualität und Verminderung der Symptomlast bei den Kindern/ Jugendlichen sowie einer geringeren Belastung für die Angehörigen Sorge zu tragen. Dieser Aspekt fand bei den stationär tätigen Pädiatern keine Berücksichtigung. Es ist davon auszugehen, dass die Verzahnung kurativer und palliativer Versorgungsmöglichkeiten im stationären Rahmen enger ist als in der ambulanten Betreuung schwerstkranker Kinder. Durch die intensive Zusammenarbeit von Mitarbeitern des Palliativteams, die selber auch einen Teil des Ärzteteams der Kinder- und Jugendmedizin Göttingen darstellen, kann vermutlich ein anderer Übergang von heilenden zu palliativen Behandlungsansätzen stattfinden. Das Hinzuziehen palliativer Versorgungsstrukturen erscheint unkomplizierter und vielleicht auch selbstverständlicher. Daneben sind bei der Entscheidung für eine palliativmedizinische Mitversorgung die unterschiedlichen Symptome einer lebenslimitierenden Erkrankung und ihre Progredienz nicht zu vernachlässigen. Als Ursache einer lebenslimitierenden Diagnose beschrieben beide der befragten Gruppen neurologische, onkologische und kardiologische Erkrankungen, die unterschiedlichste Symptome hervorrufen können. Bei der Formulierung maßgeblicher Symptome gab es unter den Aussagen der niedergelassenen und stationär tätigen Pädiater keine relevanten Unterschiede. Im Vordergrund der Behandlung stehen in einer palliativen Situation Symptome wie Schmerzen, Krampfanfälle, pulmonale Probleme (z.B. Atemnot), Ernährungs- 
probleme (z.B. Schluckstörungen), Bewusstseinsstörungen, Angst, Ödeme und Juckreiz. Hierbei ist nach Aussage einiger Pädiater eher die Symptomqualität als die Symptomquantität entscheidend.

Insgesamt ist es schwierig, einzelne detaillierte Kriterien aufzuzählen, die das Hinzuziehen einer spezialisierten pädiatrischen palliativmedizinischen Versorgungsstruktur unerlässlich machen. Laut Aussage der befragten Pädiater dieser Studie ist nicht ausschließlich das Vorliegen eines komplexen Symptomgeschehens vorrangig entscheidend. Neben der Existenz einer lebenslimitierenden Erkrankung und damit verbundenen leidvollen Symptomen, fließen in die Entscheidung über eine spezialisierte palliativmedizinische Mitversorgung laut der vorliegenden Studie auch die emotionale Bereitschaft der Angehörigen gegenüber einer solchen Versorgung und der Bedarf der Familie an kompetenter psychosozialer, pflegerischer und medizinischer Unterstützung ein. Die große Spannbreite in der Altersstruktur bei Kindern, Jugendlichen und jungen Erwachsenen mit lebenslimitierenden Erkrankungen und die unterschiedlichen Erkrankungsursachen und -Verläufe fordern bei jedem einzelnen Patienten eine sehr individuelle Einschätzung des persönlichen Versorgungsbedarfes.

\subsection{Erfassen eines idealen Zeitpunktes der Inanspruchnahme einer spezi- alisierten ambulanten palliativmedizinischen Versorgungsstruktur}

Einen übergeordnet geltenden idealen Zeitpunkt für den Beginn einer palliativmedizinischen Mitversorgung lebenslimitierend erkrankter Kinder gibt es nicht. Untersuchungen in der Erwachsenen-Onkologie geben Hinweise darauf, dass eine frühzeitige Palliativversorgung sich positiv auf die Stimmung und Zufriedenheit der Betroffenen auswirkt und zu einer Verbesserung der Gesamtversorgung der Patienten führt (SALINS et al. 2016). Studien von BAKITAS et al. (2009) und ZIMMERMANN et al. (2014) zeigen bei früher Integration von spezialisierter Palliativversorgung eine Verbesserung der Lebensqualität der Patienten. LOWERY et al. (2013) und WALKER et al. (2014) berichten von einer verbesserten Stimmung bei Patienten mit depressiven Symptomen oder Ängsten. RUGNO et al. (2014) sprechen bei früher Integration von spezialisierten palliativen Versorgungsstrukturen von einer deutlich besseren Symptomkontrolle. Grundsätzlich sind die Bedürfnisse von Erwachsenen und Kindern nicht in jeglicher Hinsicht zu vergleichen. „Kinder sind keine kleinen Erwachsenen“ - diese Aussage des Schweizer Entwicklungspsychologen Jean Piaget trifft auch auf die medizinische Versorgung von Kindern zu (SCHIESTL und STARK 2017). Dennoch ist aus Sicht der befragten Pädiater auch bei Kindern von einer positiven Auswirkung früher Integration von Palliativmedizin auszugehen. In einer nordamerikanischen, retrospektiven Umfrage von FRIEDRICHSDORF et al. (2015) wurden sechzig betroffene Eltern von bereits verstorbenen, zuvor an Krebs 
erkrankten Kindern befragt. Die Hälfte von ihnen wurde von einem ambulanten spezialisierten Palliativteam versorgt, die andere Hälfte verstarb ohne eine spezialisierte palliativmedizinische Versorgung. In der Gruppe der Kinder, die von einem ambulanten spezialisierten Palliativteam betreut wurden, konnte eine Verbesserung der Lebensqualität belegt werden und diese Kinder konnten häufiger zuhause sterben. Die Entscheidung über die Inanspruchnahme palliativmedizinischer Versorgungsstrukturen wird durch unterschiedliche Faktoren ausschlaggebend beeinflusst. Wesentliche Voraussetzung ist zunächst die Akzeptanz und Entschlossenheit der Eltern und des betroffenen Kindes, sofern es sich äußern kann, aber auch des Pädiaters bzw. des betreuenden Teams. Diese sind maßgeblich vom Verlauf der Erkrankung des Kindes abhängig. Die Zustimmung zu einer ambulanten palliativmedizinischen pädiatrischen Versorgung ist als sensibler Entwicklungsprozess innerhalb der gesamten Familie zu betrachten und die persönlichen Grenzen der Eltern in der Auseinandersetzung mit diesem Thema zu berücksichtigen. Das vorherrschende Leitbild in der Palliativversorgung ist von Fürsorge und Mitgefühl geprägt. Leidet ein Kind an einer lebenslimitierenden Erkrankung und ist die Aussicht auf Heilung nicht mehr gegeben, so ist es das Gefühl, welches das ärztliche Ansprechen palliativer Versorgungsmöglichkeiten steuert. Krankheit und Sterben sind für den Menschen unweigerlich mit Emotionen verbunden und lösen Gefühle wie Schmerz, Angst, Wut, Einsamkeit, Verzweiflung, Mitgefühl aber auch Erleichterung bei den Trauernden aus. Die enge Verknüpfung emotionaler Reaktionen mit dem Thema Tod und Sterben könnte ein Grund dafür sein, warum die befragten Pädiater sich bei der Einschätzung für den „richtigen Zeitpunkt“ einer palliativen Versorgung unter anderem von ihren Emotionen leiten lassen und auf ihr „Bauchgefühl“ vertrauen. Denkbar wäre allerdings auch, die Thematisierung von Palliativmedizin an die Diagnosestellung einer lebenslimitierenden Erkrankung oder an den Wechsel von einer kurativen zu einer palliativen Behandlungssituation zu knüpfen, ohne dass direkt eine Involvierung palliativmedizinischer Strukturen zur Unterstützung erforderlich sein muss. Auch sogenannte „red flags", also das Vorhandensein klinischer Hinweise, die eine palliative Mitversorgung sinnvoll erscheinen lassen, könnten die Thematisierung von palliativmedizinischen Unterstützungsmöglichkeiten triggern. „Red flags“ könnten eine komplexe Symptomlast, schwer zu kontrollierende belastende Symptome, rezidivierende Krankenhausaufenthalte aufgrund medizinischer Komplikationen oder die psycho-soziale Überforderung des Umfelds sein. Letztendlich sind an dieser Stelle auch die in der Verordnung spezialisierter ambulanter Palliativversorgung aufgeführten Kriterien zu nennen. Die für die Erwachsenen in der Musterverordnung (Muster 63) vorgegebenen Symptome, wie eine urogenitale Symptomatik, ausgeprägte ulzerierende/ exulzerierende Wunden oder Tumore, eine ausgeprägte Schmerzsymptomatik, eine ausgeprägte respiratorische/kardiale Symptomatik, eine ausgeprägte neurologische/psychiatrische oder psychische 
Symptomatik, eine ausgeprägte gastrointestinale Symptomatik oder ein anderes komplexes Symptomgeschehen passen auf die kindlichen Symptome nicht in allen Fällen (PALLIATIVVERSORGUNGSRICHTLINIE 2007). Als wünschenswert erachten viele der befragten Pädiater eine frühe Integration palliativer Versorgungsstrukturen im Verlauf einer lebenslimitierenden Erkrankung, wobei eine tatsächliche Definition von „früh“ nicht angegeben wird. Eine aktuelle nigerianische Studie von OLAGUNJU et al. (2016) befürwortet die frühe Integration von Palliativmedizin bei an Krebs erkrankten Kindern, um eine bessere Symptomkontrolle zum Wohle der Patienten zu erreichen. Dieser Erkenntnis entgegen steht die reale Einschätzung von befragten Pädiatern der vorliegenden Studie, dass die Inanspruchnahme einer spezialisierten palliativmedizinischen Versorgungsstruktur erst zu einem Zeitpunkt in Betracht gezogen wird, in dem es zum Eintreten einer akut lebensbedrohlichen Situation für den Patienten kommt. Dieser Zeitpunkt wird von den Befragten als deutlich zu spät eingeschätzt, um eine ideale und professionelle Versorgung lebenslimitierend erkrankter Kinder einzuleiten. Ein geltendes Merkmal für den Zeitpunkt der Inanspruchnahme einer palliativmedizinischen Versorgungsstruktur sind nach Meinung der befragten Pädiater die Häufung stationärer Aufenthalte und der Wunsch der betroffenen Familien, mit ihrem erkrankten Kind zuhause zu sein. Ein frühes Einsetzen palliativmedizinischer Versorgungsstrukturen ermöglicht aus Sicht der befragten Pädiater die individuellen Bedürfnisse eines betreffenden Kindes und der Familie besser einzuschätzen und zu berücksichtigen und gemeinsame Entscheidungen am Lebensende zu verwirklichen. Sie kann dazu beitragen, unnötige stationäre Aufenthalte zu vermeiden und Kindern und Eltern so viel Zeit wie möglich in gewohnter häuslicher Umgebung zu ermöglichen.

\subsection{Kommunikation mit der Familie}

Kommunikation (lat. communicatio, ,Mitteilung') bedeutet den Austausch oder die Übertragung von Informationen. „Information“ ist in diesem Zusammenhang eine zusammenfassende Bezeichnung für Wissen und Erkenntnis (DUDEN 2018).

Wird eine Familie mit der lebenslimitierenden Diagnose des Kindes konfrontiert, sollte im Gespräch eine gemeinsame Realität geschaffen werden, die es ermöglicht zu kommunizieren. Wichtig ist es, eine Situation herzustellen, in der nicht lediglich eine Diagnose übermittelt wird, sondern ein Dialog stattfindet. Eine schlechte Nachricht überbringt niemand gern. Nicht nur für den Patienten und seine Angehörigen ist eine schlechte Nachricht belastend, sondern sie kann auch bei dem überbringenden Arzt intensive Emotionen auslösen. Eine schlechte Nachricht ruft bei vielen Betroffenen ein kognitives, emotionales Verhaltensdefizit hervor, welches über längere Zeit persistiert (PTACEK 
und EBERHARDT 1996). Die Diagnose einer lebenslimitierenden Erkrankung des eigenen Kindes katapultiert die betroffenen Familien in eine vollkommen neue Lebenssituation, die es zu begreifen, zu akzeptieren und zu verarbeiten gilt. Ein Gesprächsrahmen, der es den Eltern erlaubt Tempo und Maß ihrer Auseinandersetzung mit der Erkrankung ihres Kindes mit zu gestalten, ist wünschenswert (RITTER 2013). Nach Meinung der befragten Pädiater ist ein Ansprechen palliativer Versorgungsstrukturen erst dann sinnvoll, wenn eine Akzeptanz der Erkrankung spürbar und die Bereitschaft der Familien erkennbar ist, professionelle Hilfe und Unterstützung anzunehmen. Die Einfühlungsgabe, den richtigen Moment für das Ansprechen palliativer Mitversorgung mit den Eltern zu erkennen, entwickele man laut der Befragten im Laufe der Berufsjahre. Die Akzeptanz einer lebenslimitierenden Erkrankung und die Aufgeschlossenheit gegenüber palliativmedizinischer Versorgungsstrukturen ist aus Sicht einiger der befragten Kinderärzte größer, wenn eine deutliche Progredienz der Erkrankung auch für die Eltern erkennbar ist. Es ist zu vermuten, dass es für die Eltern leichter ist, sich auf eine palliative Mitversorgung einzulassen, wenn sie eine Symptomverschlechterung ihrer Kinder erleben. Nicht selten geht es Kindern mit einer lebenslimitierenden Diagnose je nach Art der Erkrankung über längere Zeiträume gut und es machen sich kaum leidvolle Symptome bemerkbar (siehe auch 7.3.2.), sodass die Eltern und ggf. auch der betreuende Pädiater zunächst keine Notwenigkeit für eine palliative Mitversorgung sehen und die Vorteile einer frühzeitigen Unterstützung nicht erkennen. Die befragten Pädiater geben außerdem zu bedenken, dass das Angebot und die Inanspruchnahme spezialisierter ambulanter pädiatrischer Palliativversorgung als ein Aufgeben verstanden werden und Hoffnungslosigkeit verstärken kann. Auch nach positiven Erfahrungen mit einer frühen Integration palliativer Versorgungsstrukturen enthält „Palliativmedizin“ eine unangenehme Botschaft. Um eine erfolgreiche frühe Integration von Palliativmedizin zu verwirklichen, bedarf es einer sorgfältigen Aufklärung der Öffentlichkeit, der Patienten und der beteiligten Versorgungsstrukturen (ZIMMERMANN et al. 2016). Aufgabe hierbei ist u. a., palliativmedizinische Versorgung als hilfreiche bedarfsorientierte Unterstützung darzustellen, die mit der Hoffnung auf Symptomlinderung, Lebensqualität im Allgemeinen, Reduktion von Klinikaufenthalten etc. verknüpft ist. Von den befragten Pädiatern wurde ein möglichst frühzeitiges Ansprechen von Palliativmedizin empfohlen, um die Lebensqualität der Patienten zu erhalten und zu verbessern. Spezialisierte ambulante pädiatrische Palliativversorgung kann wesentlich zu einer besseren Pflege und ihrem Wohlbefinden beitragen (NISWANDER 2014). Hierzu bedarf es klarer Kriterien, die über das reine „Bauchgefühl“ hinausgehen. 


\subsection{Umgang mit der eigenen Rolle als niedergelassener oder stationär tä- tiger Pädiater im Rahmen der palliativen Patientenversorgung}

Im Umgang mit der eigenen Rolle im Rahmen der palliativen Patientenversorgung gibt es aus Sicht der niedergelassenen und stationär tätigen Pädiater deutliche Unterschiede. Die Mehrzahl der niedergelassenen Pädiater beschreiben kaum eigene Erfahrungen mit lebenslimitierend erkrankten Kindern. In der eigenen Praxis betreuen sie nur selten Kinder mit einer lebenslimitierenden Erkrankung. Die Seltenheit der Begleitung lebenslimitierend erkrankter Kinder durch die niedergelassenen Pädiater im Raum Göttingen ist nachvollziehbar. Kinder sind sehr selten von einer lebenslimitierenden Erkrankung betroffen und ein niedergelassener Pädiater ist somit kaum mit lebenslimitierend erkrankten Kindern und ihren Familien konfrontiert. Zudem bestehen in der Stadt Göttingen mit der Klinik für Kinder- und Jugendmedizin und dem Palliativzentrum Göttingen qualifizierte palliativmedizinische Versorgungsstrukturen, die die fachgerechte Behandlung und Betreuung von lebenslimitierend erkrankten Kindern sicherstellen. Oft wird die lebenslimitierende Diagnose erst nach ausführlicher Diagnostik in der Klinik gestellt und dort auch von den stationär tätigen Pädiatern mit den Familien kommuniziert und das weitere Vorgehen besprochen. Dies ist sicher einer der Gründe, warum niedergelassene Kinderärzte weniger Berührungspunkte mit lebenslimitierend erkrankten Kindern haben. Die Versorgung lebenslimitierend erkrankter Patienten wird mit dem Wissen, dass es dort spezialisierte Versorgungsmöglichkeiten gibt, mitunter den stationär tätigen Pädiatern bzw. dem ambulanten Palliativteam überlassen. Unsicherheit im Umgang mit den betroffenen Familien kann zu einem Vermeidungsverhalten führen. Fühlen sich Ärzte unwohl beim Überbringen schlechter Nachrichten, kann dies dazu führen, dass sie entsprechenden Situationen ausweichen [...] (TESSER 1971). Neilson beschreibt, dass mangelnde Vertrautheit mit einer Familie, sowie die Seltenheit von lebenslimitierend erkrankten Kindern in der Praxis, Unsicherheit bei niedergelassenen Pädiatern im Umgang mit den betroffenen Familien hervorrufen kann (NEILSON 2015). Ein weiterer Aspekt, der sicherlich eine übergeordnete Rolle spielt ist, dass die Aufklärung, Anleitung und Begleitung einer Familie mit einem schwerstkranken Kind ein hohes Maß an Flexibilität und Zeitaufwand bedeutet, den ein niedergelassener Pädiater in seiner Praxis kaum leisten kann. Bei Diagnosestellung einer lebenslimitierenden Erkrankung in der Klinik übernehmen die stationär tätigen Pädiater die Aufklärung und Beratung der betroffenen Familien. Je nach klinischem Zustand des Patienten wird die Indikation für eine palliativmedizinische Versorgung gestellt und gemeinsam mit den Eltern eine mögliche Inanspruchnahme besprochen. Eine Kontaktaufnahme mit dem Palliativteam ist bereits während des stationären Aufenthaltes möglich, um eine palliative Mitversorgung im häusli- 
chen Umfeld möglichst übergangslos zu gewährleisten. Aus der Sicht der stationär tätigen Pädiater gelingt eine positive und professionelle Zusammenarbeit mit den Mitarbeitern des Palliativteams.

\subsection{Erfahrungen mit Palliativmedizin}

Persönliche Erfahrungen mit Palliativmedizin werden von den niedergelassenen Pädiatern als eher selten angegeben (siehe 7.3.5). Insgesamt bewerten aber sowohl niedergelassene als auch stationär tätige Pädiater die Erfahrungen - wenn vorhanden - mit palliativen Versorgungsstrukturen als positiv. Sie beschreiben den Eindruck, dass sich sowohl Eltern als auch Patienten gut betreut, entlastet und aufgehoben fühlen. Diese Ansicht deckt sich auch mit der Bewertung befragter Familien zur Einführung der spezialisierten ambulanten Palliativversorgung in einer Untersuchung von KREMEIKE et al. (2016). In dieser Studie wurden zwanzig semistrukturierte Interviews mit Eltern von lebenslimitierend erkrankten Kindern in Niedersachsen geführt. Dreizehn der befragten Familien hatten Erfahrung mit spezialisierter ambulanter pädiatrischer Palliativversorgung, sieben von ihnen nahmen keine spezialisierte ambulante pädiatrische Palliativversorgung in Anspruch. Die Eltern mit Erfahrung in der spezialisierten ambulanten pädiatrischen Palliativversorgung bewerteten den Nutzen der häuslichen Versorgung, die Versorgungskontinuität und die Leistungsbringer als Team positiver als die Familien ohne eine spezialisierte ambulante pädiatrische Palliativversorgung. Eine mögliche Erklärung hierfür könnte sein, dass Familien mit persönlichen Erfahrungen in der spezialisierten ambulanten pädiatrischen Palliativversorgung initial bestehende Vorurteile und Ängste, Palliativversorgung könnte ein „Aufgeben“ signalisieren, ablegen konnten und die Vorteile einer solchen Versorgung im Vordergrund standen. Aus Sicht der in dieser Studie befragten Pädiater wird die Zusammenarbeit mit dem Palliativteam als strukturiert, organisiert und kommunikativ beschrieben und die permanente Verfügbarkeit kompetenter Ansprechpartner wird als äußerst positiv erachtet.

\subsection{Bedeutung ambulanter pädiatrischer Palliativversorgung aus Sicht der befragten Pädiater}

Die spezialisierte ambulante pädiatrische Palliativversorgung stellt die Möglichkeit dar, eine professionelle ambulante Versorgung schwerstkranker Kinder und ihrer Familien zu gewährleisten. Dazu zählt die pflegerische, medizinische, psychosoziale und spirituelle Betreuung der betroffenen Familie in ihrem gewohnten Umfeld verbunden mit einer kontinuierlichen Erreichbarkeit des ambulanten Palliativteams. Eine amerikanische Studie aus dem Jahr 2014 ergab, dass Kinder, die an einer komplexen chronischen Erkrankung versterben, die letzten sechs Monate zuhause verbringen und in dieser Zeit von einer 
palliativen Mitversorgung profitieren (NISWANDER et al. 2014). Ziel ist hierbei, den Kindern einen annähernd normalen Alltag in vertrauter Atmosphäre zu ermöglichen, leidvolle Symptome zu lindern und die Lebensqualität der Kinder zu erhalten und zu verbessern. Die palliativmedizinische Mitversorgung eines schwerstkranken Kindes scheint sich positiv auf die Lebensqualität auszuwirken und macht ein Sterben zuhause wahrscheinlicher (FRIEDRICHSDORF et al. 2015). Pädiatrische Palliativmedizin bedeutet für die Befragten auch, Sterben zuzulassen und Kinder gemeinsam mit ihren Familien unter Beachtung ihrer individuellen Bedürfnisse in Würde auf ihrem Weg zu begleiten. Auch über den Tod des Kindes hinaus gewährleistet die spezialisierte ambulante pädiatrische Palliativversorgung die Nachsorge und Trauerbegleitung der Familien.

\subsection{Wünsche}

Der Gedanke einer spezialisierten pädiatrischen Palliativversorgung stellt einen neuen, wachsenden und sich weiterentwickelnder Bereich in der Medizin dar. Es wurde deutlich, dass die befragten Kinderärzte diese Entwicklung befürworten und das Bedürfnis verspüren, sich in diesem Bereich regelmäßig fort- und weiterzubilden. Die Betreuung palliativmedizinisch zu versorgender Patienten wird als wesentlicher Bestandteil des Facharztkataloges gesehen und ein selbstverständliches Miteinander von kurativer und palliativer Medizin als wünschenswert formuliert. Möglicherweise wird dieses deshalb besonders erwähnt, da der Umgang mit der Gegenwart des Todes und die Akzeptanz des Sterbens einigen Ärzten schwerfallen und der Tod von behandelnden Medizinern auch als Niederlage empfunden werden kann. Die Übermittlung einer lebenslimitierenden Diagnose muss nicht nur für den Patienten, sondern kann auch für den überbringenden Arzt eine belastende Situation darstellen. Gerade für junge Mediziner stellt dies oft eine Herausforderung dar. Aus diesem Grund gehört laut Aussage der Befragten die Auseinandersetzung mit Sterben und Tod zur Ausbildung und zum Beruf des Pädiaters dazu. Inzwischen wird die Gesprächsführung bei Ärzten (BUCKMAN 1992; MAGUIRE und PITCEATHLY 2002; MILLER und ROLNICK 2002) und bei Medizinstudenten mit Hilfe von standardisierten, vorgegebenen Patientenrollen oder Schauspielpatienten geschult (TERZIOGLU et al. 2003). Bei den befragten Pädiatern zeigten sich Kompetenzen und Arbeitsstrategien vorrangig durch individuelle alltägliche Erfahrung geprägt. Interessant in diesem Zusammenhang wäre die Frage, ob und wie sich der Lehrplan der Studenten auf die Kompetenzbildung in Bezug auf Gesprächsführung und den Umgang mit Sterbenden niederschlägt. Als Idealfall einer spezialisierten pädiatrischen Palliativversorgung beschrieben die Befragten das Vorhandensein einer Kinderpalliativstation oder von „Palliativbetten“, wie eines (mittlerweile zwei) in der Kinderklinik in Göttingen verfügbar 
ist. Ein weiteres Anliegen war den befragten Pädiatern die Gewährleistung einer flächendeckenden Versorgung lebenslimitierend erkrankter Kinder und die nötige Zeit sich mit den Belangen dieser Kinder und ihrer Familien zu beschäftigen. Zwingend erforderlich hierfür ist eine Verbesserung der organisatorischen Abläufe mit ausreichenden personellen und finanziellen Möglichkeiten, um den Anforderungen dieser Aufgabe gerecht werden zu können. Weiterhin sollten sowohl stationär tätige als auch niedergelassene Pädiater für Palliativmedizin sensibilisiert werden und weitere Verbesserungen in organisatorischen und strukturellen Bereichen erreicht werden, um bestehende Möglichkeiten intensiv umsetzen zu können.

\subsection{Ausblick}

Ziel der Sozialforschung ist die möglichst unverfälschte Erfassung der sozialen Wirklichkeit. (LAMNEK 1995). Qualitative Methoden ermöglichen die Untersuchung subjektiver Wirklichkeiten, subjektiver Sinnkonstruktionen und Alltagstheorien. Das ermöglicht, Lebenswelten von innen heraus zu beschreiben und individuelle Sichtweisen und Meinungen oder Motive zu analysieren. (MISOCH 2015). Indem sich der Wissenschaftler auf die Perspektive des Befragten einlässt, kann er neue Aspekte entdecken, die mit quantitativen Verfahren kaum zu ermitteln wären (KVALE 1996). Daher ist in der palliativmedizinischen Forschung neben deskriptiv-statistischen Verfahren auch der Einsatz qualitativer Forschungsmethoden sinnvoll. Die Durchführung semistrukturierter Interviews in dieser Studie konnte dazu beitragen die persönliche Sichtweise von niedergelassenen und stationär tätigen Pädiatern zum Thema Palliativmedizin in der Pädiatrie abzubilden. Da dem Interviewten eine gewisse Gestaltungsfreiheit bezogen auf die Inhalte ermöglicht wird, sind persönliche Befragungen eine geeignete Form der Datenerhebung auf sensiblen Gebieten (HYNSON et al. 2006). Die vergleichsweise allgemeinen Ergebnisse unserer Studie entsprechen den Erwartungen bei der Befragung von Pädiatern, die eine sehr heterogenen Patientengruppe betreuen, die sich aus dem unterschiedlichen psychosozialen und kognitiven Entwicklungsspektrum, unterschiedlichen Versorgungsgruppen und Diagnosen sowie einem individuell geprägten, komplexen Symptomgeschehen ergeben (ZERNIKOW und NAUCK 2008). Forschung im Bereich Palliativmedizin widmet sich einer besonders vulnerablen Patientengruppe in einer sehr besonderen Phase des Lebens (GYSELS et al. 2013), so dass die Planung und Durchführung von Forschungsprojekten in diesem Bereich eine besondere Verantwortung darstellt. Eine weitere Herausforderung stellt hierbei die Forschungsarbeit in der pädiatrischen Palliativmedizin dar, da Patienten selber häufig nicht befragt werden können und ihre Kommunikationsmöglichkeiten eingeschränkt sind und somit viele Ergebnisse von Studien auf der Sicht- 
weise und Meinung der Eltern oder der Betreuenden basieren. Palliativmedizinische Forschung in der Pädiatrie ist notwendig, um Behandlungs- und Versorgungsmöglichkeiten zu entwickeln, die speziell auf die Bedürfnisse von Kindern angepasst sind. Dabei interessieren Fragestellungen zur Versorgungsstruktur, zur Symptomerfassung, zu medikamentösen und nicht-medikamentösen Behandlungsmöglichkeiten, zur Rolle der Behandelnden und der Familien sowie zu Patienten bezogenen Merkmalen wie Alter oder Art der Erkrankung. In einem 2014 erschienenen Artikel im Journal of Pediatric Nursing heißt es, dass Forschung im Bereich der pädiatrischen Palliativmedizin zukünftig qualitativ hochwertigere Studien mit größeren Stichprobengrößen und Kontrollgruppen beinhalten sollte. Die Perspektive der Kinder - sowohl von Patienten als auch von Geschwistern - sollte hierbei miterfasst werden, um eine allumfassende Verbesserung der Lebensqualität der Patienten zu ermöglichen. Um dies zu gewährleisten, schreiben O'Quinn und Giambra (2014), sei ein zuverlässiges Instrument erforderlich, welches es erlaube spirituelle Elemente und spezielle Bedürfnisse von Kindern mit einer lebenslimitierenden Erkrankung einzubeziehen. Die zukünftige Forschung mit diesem Werkzeug werde dazu beitragen, Fragen vollständig zu beantworten und die Lebensqualität der Kinder mit Hilfe professioneller Palliativteams zu verbessern. Qualitative Forschungsmethoden bieten hier einen Ansatz, einige der offenen Fragen zu beantworten und grundlegende Forschungsergebnisse zu liefern, indem die Bedürfnisse von lebenslimitieren erkrankten Kindern und ihren Familien vollständiger wahrgenommen werden können. Insgesamt könnte so eine Verbesserung der Lebensqualität lebenslimitierend erkrankter Kinder erreicht werden und damit zu einer Verbesserung der Gesamtsituation in der Familie beitragen. Um Kriterien für die frühe Integration von Palliativmedizin in der Pädiatrie noch genauer eingrenzen zu können, wäre es erstrebenswert, mehr über die Sichtweisen der Eltern bezüglich der Inanspruchnahme spezialisierter ambulanter pädiatrischer Palliativversorgung zu erfahren und Ergebnisse aus der Evaluation palliativer Mitversorgung aus der Perspektive der Eltern und ggfls. Geschwisterkinder mit in eine Studie einfließen zu lassen. Auch wäre es sinnvoll zu untersuchen, ob die Art einer Erkrankung, der zu erwartende Verlauf oder das Alter eines Kindes bei Diagnosestellung Einfluss auf die Inanspruchnahme palliativer Versorgungsstrukturen haben könnte. Der Effekt einer spezialisierten ambulanten pädiatrischen Palliativversorgung wirkt sich positiv auf die Lebensqualität der Betroffenen aus und ermöglicht den Patienten häufiger ein Sterben zuhause (FRIEDRICHSDORF et al. 2015). Eine frühzeitige spezialisierte palliativmedizinische Mitbehandlung pädiatrischer Patienten im ambulanten Bereich verbessert ihre Mobilität, Autonomie und die Lebensqualität (TADMOR et al. 2003). 


\section{Zusammenfassung}

Die vorliegende Studie hatte zum Ziel, Kriterien für die (frühe) Integration von Palliativmedizin in der Pädiatrie aus Sicht niedergelassener und stationär tätiger Pädiater zu ermitteln. Zu diesem Zweck wurden nach der Erfassung personenbezogener Daten der Teilnehmer mittels eines eigens erstellten Fragebogens zwanzig Pädiater interviewt. Zehn von ihnen waren in einer Kinderarztpraxis tätig, zehn weitere im Krankenhaus beschäftigt. Im Rahmen der Untersuchung fanden semistrukturierte Einzelinterviews statt, die sich an einem zuvor erstellten Interviewleitfaden orientierten. Das Interviewmaterial wurde anschließend wortgetreu transkribiert und die Texte mithilfe des Verfahrens der qualitativen Inhaltsanalyse nach Mayring zusammengefasst. Dabei wurde induktiv vorgegangen. Das durch Zusammenfassung (Paraphrasierung, Generalisierung, 1. Reduktion, Generalisierung, 2. Reduktion) entstandene Kategoriensystem wurde daraufhin nochmals am Ausgangsmaterial rücküberprüft. Ein häufig zitierter Vorteil der Qualitativen Inhaltsanalyse ist, dass sie schnell und einfach durchzuführen sei (LAMNEK 2005). Besonders bei der hier verwendeten induktiven Kategorienbildung wurden jedoch bis zur Erstellung des endgültigen Kategoriensystems unterschiedliche Vorgehensweisen (MAXQDA und händisch mit Tabellen) angewendet sowie in mehreren Durchgängen verschiedene Varianten von Kategoriensystemen erarbeitet und überprüft, so dass die Auswertung und Analyse des Materials insgesamt nicht wenig Zeit und Geduld beansprucht haben. Um subjektive Wahrnehmungen, Empfindungen und Erfahrungen der Befragten abzubilden, stellt die qualitative Inhaltsanalyse nach Mayring ein empfehlenswertes Instrument dar.

Die Betreuung lebenslimitierend erkrankter Kinder und ihrer Familien ist wichtiger Bestandteil der beruflichen Tätigkeit des Pädiaters, jedoch kommen niedergelassene Pädiater seltener als stationär tätige Pädiater mit schwerstkranken Kindern und ihren Familien in Kontakt. Anstelle der Versorgung in der Klinik ist eine Betreuung der Patienten in ihrem vertrauten Umfeld zu begrüßen. Häufig stellt dies einen intensiven Wunsch des Patienten und der Familie dar. Eine weitere Förderung der ambulanten spezialisierten pädiatrischen Palliativversorgung wäre unbedingt wünschenswert. Denkbar wäre eine größere Einbindung niedergelassener Pädiater, eine engere Zusammenarbeit mit den spezialisierten Palliativteams für Kinder, Jugendliche und junge Erwachsene und die persönliche Begleitung lebenslimitierend erkrankter Kinder auch im niedergelassen Bereich.

Die Diagnose einer lebenslimitierenden Erkrankung bringt für den behandelnden Pädia- 
ter zahlreiche Herausforderungen mit sich. Insbesondere zu nennen sind hier die Übermittlung der Diagnose einer lebenslimitierenden Erkrankung und das Ansprechen einer möglichen Einbindung palliativer Versorgungsstrukturen. Stimmt die betroffene Familie einer spezialisierten ambulanten palliativen Mitversorgung zu, kommen organisatorische Aufgaben hinzu. Kind und Familie werden im Idealfall bis über den Tod hinaus unterstützt und begleitet.

Unter den Befragten zeigte sich erhebliches Interesse und große Aufgeschlossenheit hinsichtlich dieser Studie. Alle Teilnehmer wiesen ein Grundverständnis für pädiatrische palliativmedizinische Versorgungsstrukturen in ihrer Umgebung auf und waren über die Existenz des Kinderpalliativzentrums Göttingen informiert. Ergänzende Fortbildungsmaßnahmen wurden von den Befragten gewünscht.

Da für jeden Patienten individuell sehr unterschiedliche Faktoren in die Entscheidung für eine spezialisiere Palliativversorgung mit einfließen, ließ die Befragung der Kinderärzte eine eindeutige Festlegung allgemeingültiger Merkmale für die Integration von Palliativmedizin in der Pädiatrie nicht zu. Grundsätzlich können die im Folgenden genannten Punkte als Kriterien verstanden werden, die von niedergelassenen und stationär tätigen Pädiatern hinsichtlich einer palliativen Versorgung ihrer Patienten als wichtig erachtet werden. Voraussetzung für das Hinzuziehen einer spezialisierten ambulanten pädiatrischen Versorgung ist das Vorhandensein einer lebenslimitierenden Erkrankung. Zudem sind die Akzeptanz und Bereitschaft der Eltern und des Patienten als erforderlich anzusehen. Die Notwendigkeit einer spezialisierten Versorgung muss eindeutig erkennbar sein, etwa aufgrund von Überforderung und hoher Belastung der gesamten Familie und/ oder unzureichender Möglichkeiten der ambulanten Versorgung aufgrund hoher Symptomlast und/oder komplexem Symptomgeschehen. Eine entscheidende Rolle spielen vor allem der Zustand des Patienten, das Fortschreiten der Erkrankung und die damit verbundenen leidvollen Symptome. Ergänzend zu den in $\S 2$ der Richtlinien des Gemeinsamen Bundesausschusses zur Verordnung von spezialisierter ambulanter Palliativversorgung (SAPV-RL) geregelten Voraussetzungen, die neben dem Vorliegen einer nicht heilbaren Erkrankung das Vorhandensein eines komplexen Symptomgeschehens beschreiben, fließen laut Aussage der Befragten dieser Studie auch Aspekte einer grundsätzlichen Bereitschaft und Offenheit der Familie gegenüber palliativer Mitversorgung und die Einschätzung eigener Ressourcen sowie auch das Erreichen von persönlichen Kompetenzgrenzen des Pädiaters in den Entscheidungsprozess für eine spezialisierte ambulante pädiatrische Palliativversorgung mit ein. Der ideale Zeitpunkt für das Ansprechen von palliativer Mitversorgung ist also individuell unterschiedlich. Viele der 
befragten Pädiater äußerten jedoch, dass sie einen frühen Zeitpunkt palliativer Integration prinzipiell befürworten.

Ziel der spezialisierten ambulanten pädiatrischen Palliativversorgung ist eine professionelle ambulante Versorgung schwerstkranker Kinder und ihrer Familien mit entsprechender pflegerischer, medizinischer, psychosozialer und spiritueller Betreuung. Sie trägt dazu bei, leidvolle Symptome zu lindern und die Lebensqualität der Kinder zu erhalten und zu verbessern. Das gewohnte Umfeld bietet hierbei eine vertraute Atmosphäre und die Möglichkeit, so lange wie möglich am Altag der Familie teilzunehmen. Häufige Krankenhausaufenthalte können mit professioneller Unterstützung vermieden werden und ein Abschied im häuslichen Umfeld wird möglich.

Diese Studie gibt Hinweise darauf, dass qualitative Forschungsmethoden im Bereich der Palliativmedizin einen neuen Erkenntnisgewinn bringen können, den quantitative Daten gerade bei der Erfassung von Sichtweisen und persönlichen Erfahrungen nicht zwangsläufig ermöglichen. Eine Intensivierung der Forschung in der Palliativmedizin sollte sich mit den speziellen Bedürfnissen von Kindern und deren Familien, sowie der Erfassung von Symptomen, Prozessen der Entscheidungsfindung und bestehenden Versorgungsstrukturen und deren Vorgehensweise beschäftigen, um eine flächendeckende, professionelle und evidenzbasierte Palliativversorgung für Kinder, Jugendliche und junge Erwachsene und ihre Familien gewährleisten zu können.

In einem Netzwerk flächendeckender spezialisierter pädiatrischer Palliativversorgung nehmen sowohl niedergelassene als auch stationär tätige Pädiater eine entscheidende Rolle ein. Neben einer gesetzlichen Verankerung stellt eine ausreichend geregelte strukturelle, administrative, monetäre und ideelle Förderung aller Bereiche dieses Netzwerkes eine wichtige Motivation dar, um persönliches Engagement in die Versorgung lebenslimitierend erkrankter Kinder einzubringen. Das Anliegen der Palliativmedizin in der Pädiatrie sollte im klinischen Alltag weiterhin Berücksichtigung finden, stärker im beruflichen Selbstverständnis verankert sein und weiter gefördert werden. 


\section{Anhang}

\subsection{Kooperationserklärung}

A.nlege Kooperation mit der Klinık fur Kin:Jer und Jugendmedizln

Georg-August-Universität Göttingen

Abte lung Paliativmedizin

Abte lungsdircktcr: Prof. Dr. Fredemann Nauck

Wissenzchofiliche Studienlcitung:

Dr. med. Gesine Benze

Robert-Koch-Str. 1C, 37075 Gottingen

Telefon: :0551) 39-10501

\section{$\begin{aligned} \text { LNIVERSITÄTSMEDIZIN } & =1 / \mathbf{M} \\ \text { COTTINGEN } & =\mathbf{O}\end{aligned}$}

Kooperationserklärung

Das Projekt der Klinik für Pallistumedizh der Unive sittatemesizin Gotingen rit sem Tiel ,Kriterien für die frjh Intedration wor Paliativmedizn in de- Padiatriz - Welche Kriterien erazhten n edergelasscrie und slationar larige ragdater als relevant fiit tie Zuweisung ihrer Pattenten zu ener spezialisierten pădiatnschen pallativen Versorgung?" wird von der Kinik fur Kinder- und Jugendmedizn der Universilätsmedizin zälingen unterstutzt.

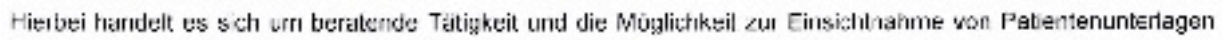
der Kirder, die eine spezialisierte ambulanle Pallialiwersorgung erhiehen. Die Auswertung der Daten iAlter. Ges:hlectt. Haupt- und Nebendiagnosen. Symfiome, Enternung des Wohnortcs nach Gxtlingen Anzahl der Kontakte zum SAPP'-Team) erfolgt in anonymisierter Form.

\footnotetext{
$10.9 \cdot 18$ Datum
}

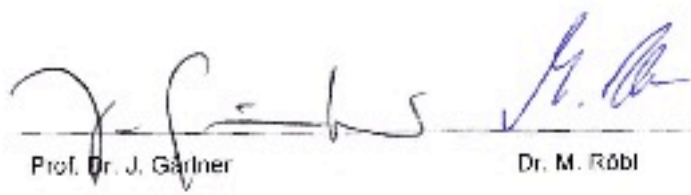




\subsection{Ethikvotum}

\section{UNIVERSITÄTSMEDIZIN
GÖTTINGEN}

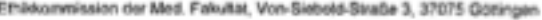

\section{Herrn}

Prof. Dr. med. Friedemann Nauck

Klinik fur Palliativmedizin

Zentrum Anasthesie, Rettungs- und

Intensivmedizin

Robert-Koch-Straße 40

worab per E-Ma:

palliativmedizinemed uni-goettingen de
Medizinische Fakultat

Ethikkommission

Vorsitzender: Prot. Dr. Jürgen Brockmoller Referentin Regierungsratin Doris Wettschereck 0551/39-8644 Telefon

Von-Siebold-Straße 3, 37075 Gottingen

Adresse

0551 / 39-6629 Telefon $0551 / 39-9536$ Fax

ethikemed.uni-goettingen.de E-Mail www.ethikkommission.med.uni-goettingen.de

15.10.2013 br - zi - go Datum
Antragsnummer: $\quad 7 / 7 / 13$ (bitte stets angeben)

Studientitel: $\quad$ Kriterien for die frohe Integration von Pallativmedicin in der Padiatrie - Welche Kriterien erachten niedergelassene und stationar tatige Padiater als relevant för die Zuweisung ihrer Patienten zu einer spezialisierten padiatrischen pallativen Versorgung?

Antragsteller: $\quad$ Prof. Dr. med. Friedemann Nauck, Dr. med. Gesine Benze, Klini für Palliativmedizin, Zentrum Anàsthesie, Retlungs- und Intensivmedizin, Prof. Dr, med. Jutta Gärtner, Dr. med. Markus Robel, Klinik for Kinder- und Jugendmedizin, Abt. Neuropadiatrie, UMG

Doktorandin: Inga Henneke

Folgende Unterlagen wurden zur Bewertung nachgereicht:

- Anschreiben nebst Eributerungen vom 19.09.2013

- Oberarbeitetes Studienprotokoll

- Oberarbeilete Befragungsbögen (Teil 1 und Teil 2)

- Oberarbeitete Informationsschrift

- Kooperationsvereinbarung

Sehr geehrter Herr Prof. Dr. Nauck, sehr geehrte Damen und Herren,

nach Ergänzung der o.g. Dokumente und Beantwortung der im vorlaufigen Votum aufgefuhrten Fragen in ihrem Schreiben vom 19.09.2013 bestehen nunmehr keine ethischen und rechtlichen Bedenken gegen die Durchfärung des oben genannten Forschungsworhabens.

Wir wönschen Ihnen viel Erfolg bei der Durchfohrung Ihres Projektes.

Unabhängig vom Beratungsergebnis macht die Ethik-Kommission daraut autmerksam, dass die ethische und rechtliche Verantwortung für die Durchfuhrung einer wissenschaftichen Studie beim verantwortlichen Studienarzt und aller an der Studie beteiligten Arzte liegt.

Alle Annderungen im Studienprotokoll müssen der Ethik-Kommission vorgelegt werden und düren erst nach der zustimmenden Bewertung umgesetzt werden.

Ober alle schwerwiegenden unerwarteten unerwünschten Ereignisse, die während der Studie auftreten und die Sicherheit der Studienteilnehmer oder die Durchfünung der Studie beeinträchtigen kőnnten, muss die EthikKommission unterrichtet werden.

Der Abschluss/Abbruch der Studie ist mitzuteilen und ein AbschlluBbericht vorzulegen. 


\section{UNIVERSITĀTSMEDIZIN \\ COTINGEN UMG}

Auf die Einhaltung einschlägiger Gesetze und Rechtsworschriften wird hingewiesen. Die nach Rechtslage notwendigen Unterrichtungen (u. A. Anderung des Studienprolokolls, Meldung von Zwischenfallen, neve Datenlage, Nachmeldung von Prützentren, Abschlussbericht) sind der Ethik-Kommission unverzbglich vorzulegen

Die Ethik-Kommission bestätigt, dass sie auf Grundlage nationaler Gesetze, Vorschriften sowie der GCP/CH-Richtlinie arbeitet.

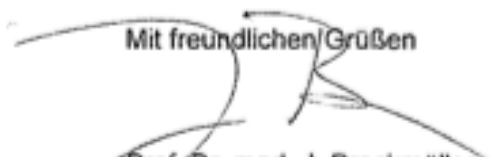

Prok. Dr. med. J. Brockmoller

Vorsitzender der Ethik-Kommission 


\subsection{Information und Aufklärung für die Teilnahme an einem Interview im Rahmen der Studie „Kriterien für die Integration von Palliativmedizin in der Pädiatrie“"}

\section{Georg-August-Universität Göttingen}

Abteilung Palliativmedizin

Abteilungsdirektor: Prof. Dr. Friedemann Nauck

Wissenschaftliche Studienleitung: Dr. med. Gesine Benze, Robert-Koch-Str. 40, 37075 Göttingen

\section{Information und Aufklärung für die Teilnahme an einem Interview \\ im Rahmen der Studie „Kriterien für die Integration von Palliativmedizin in der Pädiatrie“}

Sehr geehrte Frau Kollegin, sehr geehrter Herr Kollege,

im Rahmen der pädiatrischen Versorgung von Patienten können immer wieder auch palliativmedizinische Aspekte eine Rolle spielen.

Im Rahmen eines Projektes möchten wir untersuchen, welche Kriterien es für die Integration von Palliativmedizin in der Pädiatrie geben könnte. Daher möchten wir Sie einladen, an einem Interview teilzunehmen, in dem Sie die Gelegenheit erhalten, generelle Vorstellungen über dieses Thema, aber auch konkrete Beispiele aus ihrer Perspektive zu schildern.

Die Teilnahme an dieser Studie ist freiwillig, eine Nichtteilnahme bringt keinerlei Nachteile mit sich. Da in dem Interview nur wenig Fragen vorgegeben werden, hängt die Länge des Interviews davon ab, wie viel sie erzählen möchten. Es kann daher 15 Minuten oder auch länger in Anspruch nehmen. Im Vorfeld möchten wir Sie bitten, einen begleitenden Kurzfragebogen mit Daten zu Ihrer Person auszufüllen.

Für eine bessere Auswertung wird das Interview auf Tonband aufgenommen und anschließend wörtlich niedergeschrieben. Die Aufnahmen dienen lediglich dem hier beschriebenen Forschungszweck. Die Auswertung des Interviews sowie des Kurzfragebogens erfolgt pseudonymisiert. Das bedeutet, dass die Daten ohne Verwendung Ihres Namens mit Nummern kodiert werden. Die Zuordnung der Daten zu einer Person ist dann nur noch durch die verantwortlichen Studienärzte möglich, die Zugriff auf den Schlüssel haben, mit dem die Daten pseudonomisiert wurden. Die personenbezogenen Daten werden unter besonderen Schutzvorkehrungen getrennt von der Schlüsselliste aufbewahrt.

Die Studie ist Teil des Dissertationsprojektes von Frau Inga Steging. Sie wird die Interviews führen.

Aus Gründen der Qualitätssicherung werden Ihre Daten sowie die Tonbandaufnahme für einen Zeitraum von 10 Jahren unter den Bedingungen des Datenschutzes gespeichert, eine Weitergabe an Dritte ist ausgeschlossen. Sie können Ihr einmal gegebenes 
Einverständnis jederzeit ohne Angabe von Gründen zurückziehen, ohne dass Ihnen dadurch irgendwelche Nachteile entstehen; alle bis dahin erhobenen Daten würden in diesem Fall vernichtet werden.

Wir werden mit Ihnen in den nächsten Tagen telefonisch Kontakt aufnehmen, um Ihre Teilnahme an der Studie zu erfragen und um ggf. einen Interviewtermin zu vereinbaren. Die Einverständniserklärung sowie den Fragebogen können Sie entweder per Post oder per Fax (0551-393189) an uns ausgefüllt zurücksenden.

Sollten Sie noch Fragen haben, rufen Sie gerne an oder schreiben Sie ein e-Mail.

\section{Dr. med. Gesine Benze}

Oberärztin, Abteilung Palliativmedizin, Universitätsmedizin Göttingen

Robert-Koch-Straße 40, 37075 Göttingen

Tel.: (0551) 39-10501; E-Mail: gesine.benze@med.uni-goettingen.de

\section{Inga Steging}

Doktorandin 


\title{
6.4 Einverständniserklärung zur Teilnahme an einem Interview im Rah- men der Studie "Kriterien für die Integration von Palliativmedizin in der Pädiatrie"
}

\author{
Georg-August-Universität Göttingen \\ Abteilung Palliativmedizin \\ Abteilungsdirektor: Prof. Dr. Friedemann Nauck \\ Wissenschaftliche Studienleitung: Dr. med. Gesine Benze, Robert-Koch-Str. 40, 37075 Göttingen
}

\section{Einverständniserklärung zur Teilnahme an einem Interview}

im Rahmen der Studie „Kriterien für die Integration von Palliativmedizin in der Pädiatrie“

Ich, , wurde vollstän-

dig über die Durchführung des Interviews und dem damit verbundenen Kurzfragebogen im Rahmen der Studie mit dem o.g. Titel aufgeklärt. Ich hatte die Möglichkeit Fragen zu stellen, habe die Antworten verstanden und akzeptiere sie.

Mir ist bekannt, dass im Rahmen des Forschungsvorhabens personenbezogene Daten und Äußerungen erhoben werden. Ich bin damit einverstanden, dass die im Rahmen der Studie erhobenen Daten in anonymisierter Form unter den Bedingungen des Datenschutzes für einen Zeitraum von 10 Jahren gespeichert und in anonymisierter Form zu Forschungszwecken inklusive Publikationen und Vorträge verwendet werden. Die personenbezogenen Daten werden nicht an Dritte weitergegeben. Eine Anonymisierung der Daten findet statt, sobald dies nach dem Forschungszweck möglich ist. Die Tonaufnahmen dienen lediglich dem hier beschriebenen Forschungszweck; nach Beendigung der Studie werden sie nicht für weitere Studien oder im Unterricht für die Studierenden verwendet.

Ich hatte ausreichend Zeit mich zur Teilnahme an dem Interview zu entscheiden und weiß, dass meine Teilnahme freiwillig und mit keinerlei persönlichem Nutzen oder mit Nachteilen für mich verbunden ist. Ich weiß, dass ich jederzeit und ohne Angabe von Gründen diese Zustimmung widerrufen kann, ohne dass sich dieser Entschluss nachteilig für mich auswirken wird. Die bisher erhobenen Daten würden in diesem Fall vernichtet werden. 
Ich bin damit einverstanden, dass das Gespräch auf Tonband aufgezeichnet wird, und erkläre hiermit meine freiwillige Teilnahme an dem Interview.

Ich habe die Informationen zur Studie und eine Kopie der Einverständniserklärung erhalten.

Datum, Unterschrift der Teilnehmerin/ des Teilnehmers 
6.5 Kriterien für die Integration von Palliativmedizin in der Pädiatrie- Befragung niedergelassener oder stationär tätiger Pädiater, Teil 1 Basisdaten

\section{Kriterien für die Integration \\ von Palliativmedizin \\ in der Pädiatrie}

\section{Befragung niedergelassener oder stationär tätiger Pädia- ter}

\section{Teil 1 Basisdaten}

Datum der Befragung:

Informationen, die zur Identifikation von Personen führen könnten, werden nicht veröffentlicht. 
Sehr geehrte Frau Kollegin, sehr geehrter Herr Kollege,

Wir danken Ihnen für Ihre Bereitschaft, an dieser Befragung teilzunehmen und bitten Sie im Vorfeld des noch ausstehenden Interviews die unten stehenden Fragen zu beantworten.

Ihre Informationen werden vertraulich behandelt. Bei der Auswertung der Daten wird durch die Pseudonymisierung nicht ersichtlich sein, wer welche Antworten gegeben hat.

\section{A) Informationen zur Person des Pädiaters}

A1) Alter: , Geschlecht: $\square$ weiblich $\square$ männlich

A2) Jahr des Universitätsabschlusses:

A3) Weiterbildungen (Welche und wann?):

Zusatzbezeichnungen:

Palliativmedizin $\square$ ja, seit ; $\square$ nein

Weitere Zusatzbezeichnungen:

A4) Dauer der stationären Tätigkeit (in Jahren):

Dauer der ambulanten Tätigkeit (in Jahren): Jahr der Niederlassung:

\section{B) Wie sieht Ihre Patientenklientel aus?}

B1) geschätzter Altersdurchschnitt (Altersangabe):

B2) häufigste Grunderkrankungen: 
B3) geschätzte Häufigkeit eines primär palliativen (nicht-kurativen)

Behandlungsansatzes (in \%):

B4) Art der lebenslimitierenden Erkrankung (z.B. Stoffwechselerkrankung, Malig nom):

B5) Dauer der bisherigen palliativmedizinischen

Betreuung pro Patient:

C) Verfügbarkeit von (spezialisierter) pädiatrischer Palliativversorgung im Um kreis von $50 \mathrm{~km}$ (Mehrfachnennung möglich)

pädiatrische Palliativstation

spezialisierte ambulante pädiatrische Palliativversorg (SAPPV)

$\square$ Kinderhospiz $\square$ pädiatrischer Palliativpflegedienst

ambulante Hospizbewegung

andere

D) Sind Sie selbst in der spezialisierten ambulanten Palliativversorgung (SAPPV) involviert?

ja, in Form von

$\square$ nein

Sind Sie selbst in der allgemeinen ambulanten Palliativversorgung involviert?

ja, in Form von

nein 
E) Wie viel Zeit nutzen Sie persönlich in der Woche insgesamt für palliativmedizinische Aspekte? (Angabe in Stunden und Minuten) Welche Aspekte sind dies?

Wie viel Zeit für palliativmedizinische Aspekte halten Sie insgesamt für angemessen? (Angabe in Stunden und Minuten)

Welche zusätzlichen palliativmedizinischen Aspekte würden Sie gerne behandeln? 
6.6 Kriterien für die Integration von Palliativmedizin in der Pädiatrie- Befragung niedergelassener oder stationär tätiger Pädiater, Teil 2

\author{
Kriterien für die Integration \\ von Palliativmedizin \\ in der Pädiatrie
}

Befragung niedergelassener oder stationär tätiger Pädiater

\title{
Teil 2
}

Datum der Befragung:

Dauer der Befragung: Beginn:

Ende:

Minuten:

Interviewer:

Ort:

Informationen, die zur Identifikation von Personen führen könnten, werden nicht veröffentlicht. 


\section{Begrüßung und Einführung in das Interview}

(Ankündigung: Das Aufnahmegerät wird nun eingeschaltet.)

Vielen Dank, dass Sie sich zu einem Interview bereit erklärt haben. Wir interessieren uns in unserer Studie für die Integration von Palliativmedizin in der Pädiatrie. Dabei kommt es uns in unserer Studie auf ihre Perspektive und ihre Erfahrungen an. Das Interview wird ca. 15 Minuten dauern. Sie können sich aber auch gerne für Ihre Antworten mehr Zeit nehmen. Zur besseren Nachbereitung des Interviews würde ich unser Gespräch gerne aufnehmen. Ihre Daten werden pseudonymisiert und vertraulich behandelt und werden nur im Rahmen des Forschungsprojektes verwendet. Sind Sie damit einverstanden?

Haben Sie vor Beginn des Interviews noch Fragen?

Dann würde ich jetzt gerne mit dem Interview beginnen.

I) Kriterien, Krankheitsstadium

a. Sie haben im Fragebogen folgende palliativmedizinischen Versorgungsstrukturen genannt .... In welchen Fällen überweisen Sie an diese Strukturen bzw. nehmen sie in Anspruch?

b. Bei welchen Erkrankungen und Symptomen im Speziellen würden Sie palliativmedizinische Versorgungsstrukturen hinzuziehen?

c. Ist das Hinzuziehen abhängig von der Symptomstärke?

d. Gibt es einen bestimmten Zeitpunkt im Krankheitsverlauf, bei dem Sie an palliativmedizinische Mitversorgung denken?

e. Können Sie einen konkreten bzw. Ihren letzten Fall schildern, bei dem Sie eine Palliativversorgung mit einbezogen haben?

\section{II) Kommunikation}

Zu welchem Zeitpunkt thematisieren Sie gegenüber den Eltern bzw. einem Patienten eine mögliche palliativmedizinische Mitversorgung?

III) Haltung/ Einstellung

a. Wie sollte die Integration von Palliativmedizin in der Pädiatrie aus Ihrer Sicht aussehen?

b. Wann wäre für Sie der ideale Zeitpunkt für die Integration von Palliativmedizin? 
IV) Erfahrungen

Wie sehen Ihre bisherigen Erfahrungen bezüglich palliativmedizinischer Mitversorgung aus? (Was war die beste, was die schlechteste Erfahrung?)

\section{Nach dem Interview:}

Bedanken und fragen, ob man noch etwas hinzufügen möchte oder es Dinge gibt die evtl. vergessen wurden. Optional: Fragen wie das Gespräch empfunden wurde und ob Interviewpartner für ein weiteres Gespräch (wenn nötig) bereit wäre.

Dann das Interview abschließen und sich nochmal für die Teilnahme bedanken. Wenn Interview beendet Gerät ausschalten. 


\section{Literaturverzeichnis}

Adler P A, Adler P: The reluctant respondent. In: Handbook of interview research: context and method. Grubrium J F, Holstein J A (Hrsg.): Sage Publication, Thousand Oaks 2002, 515-536

Bakitas M, Lyons K D, Hegel M T, Balan S, Brokaw F C, Seville J, Ahles T A (2009): The project ENABLE II randomized controlled trial to improve palliative care for patients with advanced cancer. JAMA $\underline{302}, 741-749$

Barbour R S (2003): The newfound credibility of qualitative research? Tales of technical essentialism and cooption. Qual Health Res $\underline{13}, 1019-1027$

Bergstraesser E, Zimmermann K, Eskola K, Luck P, Ramelet A S, Cignacco E (2015): Paediatric end-of-life care needs in Switzerland: current practices, and perspectives from parents and professionals. A study protocol. J Adv Nurs $\underline{71}$, 1940-1947

Boenke U: Pädiatrische Palliativmedizinische Versorgung und präfinale Belastungen in der Kinderonkologie. Med. Diss. Unna 2009

Bohnsack R: Rekonstruktive Sozialforschung. Einführung in Methodologie und Praxis qualitativer Sozialforschung. 2. Auflage; Verlag Leske und Budrich, Opladen 1993

Bortz J, Döring N: Forschungsmethoden und Evaluation für Sozialwissenschaftler. 2. Auflage; Springer Verlag, Heidelberg 1995

Brosius H B, Koschel F: Methoden der empirischen Kommunikationsforschung. Eine Einführung. 2. Auflage; VS Verlag für Sozialwissenschaften, Wiesbaden 2001

Buckman R: How to break bad news: A guide for health care professionals. The John Hopkins University Press, Baltimore 1992

Byrne M, Tresgallo M, Saroyan J (2011): Qualitative analysis of consults by pediatric advanced care team during its first year of service. Am J Hosp Palliat Care $\underline{28}, 109-117$

Flick U: Methodologie qualitativer Forschung. In: Flick U, von Kardoff E, Steinke I (Hrsg.): Qualitative Forschung. Ein Handbuch. Rowohlt Verlag, Reinbek 2000, 252-264 
Flick U: Qualitative Sozialforschung - eine Einführung. 6. Auflage; Rowohlt Verlag, Reinbek 2002

Friedrichsdorf S J, Postier A, Dreyfus J, Osenga K, Sencer S, Wolfe J (2015): Improved quality of life at end of life related to home-based palliative care in children with cancer. J Palliat Med $\underline{18}, 143-150$

Führer M (2011): Kinderpalliativmedizin. Monatsz Kinderheilk 159, 583-596

Fuhs B: Qualitative Methoden in der Erziehungswissenschaft. Wissenschaftliche Buchgesellschaft, Darmstadt 2007

Gysels M, Evans C J, Lewis P, Speck P, Benalia H, Preston N J, Grande G E, Short V, Owens, Jones E, Todd C J, Higginson I J (2013): MORECare research methods guidance development: Recommendations for ethical issues in palliative and end-of-life care research. Palliat Med $\underline{27}$, 908-917

Helfferich C: Die Qualität qualitativer Daten. Manual für die Durchführung qualitativer Interviews. 2. Auflage; VS Verlag für Sozialwissenschaften, Wiesbaden 2005

Higginbottom G M (2004): Sampling issues in qualitative research. Nurse Res $\underline{12}$, 7-19

Honer A: Interview. In: Bohnsack R, Marotzki W, Meuser M (Hrsg.): Hauptbegriffe qualitativer Sozialforschung. Westdeutscher Verlag, Opladen 2003, 94-99

Hopf C (1987): Die Pseudo-Exploration-Überlegungen zur Technik qualitativer Interviews in der Sozialforschung. ZfS $\underline{7}, 97-115$

Hopf C: Qualitative Interviews- Ein Überblick. In: Flick U, von Kardoff E, Steinke I (Hrsg.): Qualitative Forschung. Ein Handbuch. 12. Auflage; Rowohlt Verlag, Reinbek 2005

Hospiz- und Palliativgesetz 2015: Gesetz zur Verbesserung der Hospiz- und Palliativversorgung in Deutschland in der Fassung der Bekanntmachung vom 1. Dezember 2015.

Hynson J, Aroni R, Bauld C (2006): Research with bereaved parents: a question of how not why. Palliat Med $\underline{20}, 805-811$ 
Ingleton C, Seymour J E (2001): Analysing qualitative data: examples from two studies of end-of- life-care. Int J Palliat Nurs $\underline{7}, 227-233$

Janke- Hoppe K (2004): Palliativmedizin ausbauen. Deutsche Gesellschaft für Palliativmedizin präsentiert Empfehlungen des Europarats und zeigt Handlungsbedarf in Deutschland auf. Rhein Arztebl $\underline{58}, 20$

Kaasa S, Radbruch L (2008): Palliative care research - priorities and the way forward. Eur J Cancer 44, 1175-1179

Klaschik E: Entwicklung und Stand der Palliativmedizin in Europa. In: Husebø S, Klaschik E, Jaspers B (Hrsg.): Palliativmedizin: Grundlagen und Praxis. 5. Auflage; Springer Verlag, Heidelberg 2009, 4-21

Klinkhammer G (2007): Palliativmedizin: Junge Disziplin mit großem Potenzial. Dtsch Artztebl $\underline{5}, 217-220$

Knesebeck O v d, Blum K, Grosse K, Klein J (2010): Arbeitsbedingungen und Patientenversorgung. Eine Befragung von Chirurgen und Gynäkologen zur psychosozialen Arbeitsbelastung. Arzt Krankenh $\underline{82}$, 210-213

Kohlbacher F (2006): The use of qualitative content analysisin case study research. Forum Qualitative Sozialforschung/ Forum: Qual Soc Res $\underline{7}, 21$

Kremeike K, Mohr A, Nachtmann J, Reinhardt D, Geraedts M, Sander A: Evaluation der spezialisierten ambulanten pädiatrischen Palliativversorgung in Niedersachsen - Eine qualitative Studie aus Elternsicht. Gesundheitswesen $\underline{78}, 306-312$

Kuckartz U, Dresing T, Rädiker S, Stefer C (Hrsg.): Qualitative Evaluation. Der Einstieg in die Praxis. 2. Auflage; VS Verlag für Sozialwissenschaften, Wiesbaden 2008

Kübler-Ross E: Interviews mit Sterbenden. 1. Auflage; Kreuz Verlag, Freiburg 2014

Kvale S: Interviews: An Introduction to Qualitative Research Interviewing. Sage Publications, Thousand Oaks 1996

Kvale S: Doing interviews. Sage Publications, London 2007 
Lamnek S: Qualitative Soziallforschung: Methodologie. 3. Auflage; Beltz Verlag, Weinheim 1995

Lamnek S: Qualitative Sozialforschung: Lehrbuch. 4. Auflage; Beltz Verlag, Weinheim 2005

Lotz J D, Daxer M, Jox R J, Borasio G D, Führer M (2016): "Hope for the best, prepare for the worst": A qualitative interview study on parents' needs and fears in pediatric advance care planning. Palliat Med $\underline{31}, 764-771$

Lowery W J, Lowery A W, Barnett J C, Lopez-Acevedo M, Lee P S, Secord A A, Havrilesky L (2013): Cost-effectiveness of early palliative care intervention in recurrent platinum-resistant ovarian cancer. Gynecol Oncol 130, 426-430

Mack J W and Wolfe J (2006): Early integration of pediatric palliative care: for some children, palliative care starts at diagnosis. Curr Opin Pediatr $\underline{18}, 10-14$

Maguire P, Pitceathly (2002): Key communication skills and how to acquire them. BMJ $\underline{325}, 697-700$

Mayring P. (2000): Qualitative Inhaltsanalyse. Forum Qualitative Sozialforschung/ Forum: Qualitative Social Research 1, 20

Mayring P (2001): Combination and Integration of Qualitative and Quantitative Analysis. Forum Qualitative Sozialforschung/ Forum: Qualitative Social Research 2, 6

Mayring P: Einführung in die Qualitative Sozialforschung: eine Anleitung zum qualitativen Denken. 5. Auflage; Beltz Verlag, Weinheim 2002

Mayring P: Qualitative Inhaltsanalyse: Grundlagen und Techniken. 8. Auflage; Beltz Verlag, Weinheim 2003

Mayring P: Qualitative Inhaltsanalyse. Grundlagen und Techniken, 9. Auflage; Beltz Verlag, Weinheim 2007

Mayring P: Qualitative Inhaltsanalyse. Grundlagen und Techniken. 10. Auflage; Beltz Verlag, Weinheim 2008 
Mayring P: Qualitative Inhaltsanalyse. Grundlagen und Techniken. 12. Auflage; Beltz Verlag, Weinheim 2015

Miller W R, Rollnick S (2002): Motivierende Gesprächsführung. BMJ $\underline{325}$, 697- 700

Misoch S: Qualitative Interviews. De Gruyter, Oldenbourg 2015

Monterosso L, Kristjanson L (2008): Supportive and palliative care needs of families of children who die from cancer: an Australien study. Palliat Med 22, 59-69

Müller-Busch H C: Kurze Geschichte der Palliativmedizin. In: Schnell M W und Schulz C (Hrsg.): Basiswissen Palliativmedizin. 2. Auflage; Springer Verlag, Heidelberg 2014

Nauck F, Radbruch L (2012): Evidenz in der Palliativmedizin: Auf dem Weg zur Therapieempfehlung Palliativmedizin. Schmerz $\underline{26}$, 473-474

Nauck F, Alt- Epping B, Benze G (2015): The current situation of palliative medicine in Germany- clinical implications, education and research. Anasth Intensiv Notf 50, 36-46

Neilson S J, Gibson F, Greenfield S M (2015): Pediatric Oncology Palliative Care: Experiences of General Practitioners and Berevaed Parents. J Palliat Care Med $\underline{5}, 214$

Niswander L M, Cromwell P, Chirico J, Gupton A, Korones D N (2014): End-of-life care for children enrolled in a community-based pediatric palliative care program. J Palliat Med $\underline{17}, 589-591$

Olagunju A T, Sarimiye F O, Olagunju T O, Habeebu M Y, Aina O F (2016): Child's symptom burden and depressive symptoms among caregivers of children with cancers: an argument for early integration of pediatric palliative care. Ann Palliat Med $\underline{5}, 157-65$

O'Quinn L P, Giambra B K (2014): Evidence of improved quality of life with pediatric palliative care. Pediatr Nurs $\underline{40}, 284-288,296$

Palliativversorgungsrichtlinie 2007: Richtlinie des Gemeinsamen Bundesausschusses zur Verordnung von spezialisierter ambulanter Palliativversorgung (Spezialisierte Ambulante Palliativversorgungs-Richtlinie/ SAPV-RL) vom 20. Dezember 2007 in der Fassung der Bekanntmachung vom 11. März 2008, zuletzt geändert am 15. April 2010 
Press N (2005): Qualitative research: thoughts on how to do it; how to judge it; when to use it. Genet Med $\underline{7}, 155-158$

Przyborski A, Wohlrab-Sahr M: Qualitative Forschung - ein Arbeitsbuch. 3. Auflage; Oldenbourg Wissenschaftsverlag, München 2010

Ptacek J T and Eberhardt T L (1996): Breaking bad news. A review of the literature. JAMA $\underline{276}, 496-502$

Rattay P, Starker A, Domanska O, Butschalowsky H, Gutsche J, Kamtsiuris P. KiGGS Study Group (2014): Trends in der Inanspruchnahme ambulant-ärztlicher Leistungen im Kindes- und Jugendalter. Bundesgesundheitsbl $\underline{57}, 878-891$

Ritter M: Begegnung und Begleitung im Angesicht des Todes. In: Zernikow B (Hrsg.) Palliativversorgung von Kindern, Jugendlichen und jungen Erwachsenen, 2. Auflage; Springer Verlag, Heidelberg 2013, 93-105

Rugno F C, Paiva B S, Paiva C E (2014): Early integration of palliative care facilitates the discontinuation of anticancer treatment in women with advanced breast or gynecologic cancers. Gynecol Oncol 135, 249-254

Salins N, Ramanjulu R, Patra L, Deodhar J, Muckaden M A (2016): Integration of early Specialist Palliative Care in Cancer Care and Patient Related Outcomes: A Critical Review of Evidence. Indian J Palliat Care 22, 252-257

Scheele B, Groeben N: Dialog-Konsens-Methoden zur Rekonstruktion Subjektiver Theorien: die Heidelberger Struktur- Lage- Technik (SLT), konsuale Ziel-Mittel-Argumentation und kommunikative Flußdiagramm- Beschreibung von Handlungen. Francke Verlag, Tübingen 1988

Schiestl C, Stark G B: Plastische Chirurgie bei Kindern und Jugendlichen. In: Schiestl C, Stark G B, Lenz Y, Neuhaus K (Hrsg.): Allgemeiner Teil. Springer Verlag, Heidelberg 2017, 3-118

Schikora S (2007): Klinikärzte - mehr Arbeit in kürzerer Zeit! - Chirurgen haben die höchste Arbeitsbelastung. Klinikarzt $\underline{36}, 678$ 
Schnell R, Hill P, Esser E: Methoden der empirischen Sozialforschung. 5. Auflage; Oldenbourg Verlag, München 1995

Tadmor C S, Postovsky S, Elhasid R, Ben Barak A and Arush M B (2003): Policies designed to enhance the quality of life of children with cancer at the end-of-life. Pediatr Hematol Oncol 20, 43-54

Terzioglu P, Jonitz B, Schwantes U, Burger W (2003): Kommunikative und soziale Kompetenzen. Vermittlung muss im Medizinstudium beginnen. Deutsch Arztebl 100, 36

Tesser A, Rosen S, Tesser M (1971): On the reluctance to communicate undesirable messages (the MUM effect): A field study. Psychol Rep 29 6 , 651-6544

Verberne L M, Kars M C, Schouten-van Meeteren A Y, Bosman D K, Colenbrander D A, Grootenhuis M A, van Delden J J (2017): Aims and tasks in parental caregiving for children receiving palliative care at home: a qualitative study. Eur J Pediatr $\underline{176}$, 343-354

Vollenbroich R, Duroux A, Grasser M (2012): Effectveness of a pediatric palliative home care team as experienced by parents and health care professionals. J Palliat Med $\underline{15}$, 294-300

Walker J, Hansen C H, Martin P, Symeonides S, Gourley C, Wall L, Weller D, Murray G, Sharpe M, SMaRT (Symptom Management Research Trials) Oncology-3 Teamet (2014): Integrated collaborative care for major depression comorbid with a poor prognosis cancer (SMaRT Oncology-3): A multicentre randomised controlled trial in patients with lung cancer. Lancet Oncol $\underline{15}, 1168-1176$

Weidner N, Cameron M, Lee R (2011): End-of-life care for the dying child: what matters most to parents. J Palliat Care 27, 279-286

Wolf S: Der Methodenstreit quantitativer und qualitativer Sozialforschung unter besonderer Berücksichtigung der Grundlegenden Unterschiede beider Forschungstraditionen. Bachelor-Arb. Augsburg 2008

Zernikow B (2007): IMPaCCT: Standards of Paediatric Palliative Care in Europe. J Palliat Care $\underline{14}, 109-114$ 
Zernikow B, Nauck F (2008): Kinderpalliativmedizin: Kindern ein gutes Sterben ermöglichen. Dtsch Arztebl 105, 1376-1380

Ziebertz H G: Herder Korrespondenz Spezial. Nach der Glaubensspaltung zur Zukunft des Kristentums. Heft 2; Herder Verlag, Freiburg 2016

Zimmermann C, Swami N, Krzyzanowska M, Hannon B, Leighl N, Oza A, Moore M, Rydall A, Rodin G, Tannok I et al. (2014): Early palliative care for patients with advanced cancer: A cluster-randomised controlled trial. Lancet, $\underline{383}, 1721-1730$

Zimmermann C, Swami N, Krzyzanowska M, Leighl N, Rydall A, Rodin G, Tannock I, Hannon B (2016): Perceptions of palliative care among patients with advanced cancer and their caregivers. CMAJ $\underline{188}, 217-227$ 


\section{Internetquellen}

Ambulanter Kinder- und Jugendhospizdienst Göttingen (2015): Begleitung und Unterstützung von Kindern und Jugendlichen mit lebensverkürzender Erkrankung, ihren Eltern und Geschwistern

https://www.deutscher-kinderhospizverein.de/fileadmin/pdf/Flyer/Flyer_neu/Flyer_Goettingen_2015.pdf;

abgerufen am 14.01.2017

Betreuungsnetz.Org. Netzwerk für die Versorgung schwerkranker Kinder und Jugendlicher e.V. (2017a): Spezialisierte ambulante Palliativversorgung für Kinder- und Jugendliche (SAPV-KJ) https://www.betreuungsnetz.org/spezialisierte-ambulante-paediatrische-palliativversorgung-sapv-kj/;

abgerufen am 14.01.2017

Betreuungsnetz.Org. Netzwerk für die Versorgung schwerkranker Kinder und Jugendlicher e.V. (2017b): Koordinierungsbüro

https://www.betreuungsnetz.org/koordinierungsbuero-fuer-niedersachsen/; abgerufen am 14.01.2017

Betreuungsnetz.Org. Netzwerk für die Versorgung schwerkranker Kinder und Jugendlicher e.V. (2017c): Regionalteam

https://www.betreuungsnetz.org/spezialisierte-ambulante-paediatrische-palliativversorgung-sapv-kj-regionalteam-braunschweig/...;

abgerufen am 14.01.2017

Braun (2015): Fachwissen Palliative Care - Geschichte

http://www.palliativecare.bbraun.de/cps/rde/xchg/om-palliativecare-dede/hs.xsl/7216.html;

abgerufen am 16.03.2015

Bundesverband Kinderhospiz e.V. (2017): Übersichtskarte aller stationären Kinderhospize

http://www.bundesverband-kinderhospiz.de/übersichtskarte-aller-stationären-kinderhospize-in-betrieb;

abgerufen am 14.01.2017 
Charta Bundesärztekammer. Deutsche Gesellschaft für Palliativmedizin e. V., Deutscher Hospiz- und Palliativverband e. V., Bundesärztekammer (Hrsg.): Charta zur Betreuung schwerstkranker und sterbender Menschen in Deutschland. 8. Auflage; o.Verl., o.O. 2015

https://www.bundesaerztekammer.de/fileadmin/user_upload/downloads/pdf-Ordner/Hospiz/charta_broschuere.pdf abgerufen am 09.09.2017

Deutsche Gesellschaft für Palliativmedizin (2015): Wegweiser Hospiz- und Palliativversorgung Deutschland. Übersicht zum aktuellen Stand der Hospiz- und Palliativversorgung in Deutschland https://www.dgpalliativmedizin.de/images/stories/pdf/151026_Wegweiserübersicht_Homepage_Kinder.pdf; abgerufen am 21.03.2016

Deutscher Hospiz- und Palliativverband e. V.: Stationäre Hospize für Erwachsene, Stationäre Hospize für Kinder, Jugendliche und junge Erwachsene sowie Palliativstationen in Deutschland - Daten zur Entwicklung und zum aktuellen Stand vom 11.04.2016 http://www.dhpv.de/service_zahlen-fakten.html;

abgerufen am 20.03.2017

Deutsche Krebshilfe (2015): Dr. Mildred Scheel Akademie - Gründung https://www.krebshilfe.de/helfen/seminare-kurse/dr-mildred-scheel-akademie/; abgerufen am 16.03.2015

DKHV e.V. (Deutscher Kinderhospizverein) (2017): Geschichte https://www.deutscher-kinderhospizverein.de/der-verein/; abgerufen am 20.03.2017

Duden (2018): Begriffserklärung Information https://www.duden.de/rechtschreibung/Information; abgerufen am 20.04.2018

Gesundheitsberichterstattung des Bundes (2018): Zahl der Mediziner mit einer Zusatzausbildung für Palliativmedizin https://www.dhpv.de/service_zahlen-fakten.html abgerufen am 20.04.2018 
Kinder- und Jugendhospiz Balthasar (2015a): Idee und Geschichte http://www.kinderhospiz-balthasar.de/kinderhospiz/idee-und-geschichte.html; abgerufen am 20.03.2015

Kinder- und Jugendhospiz Balthasar (2015b): Unser Haus https://www.kinderhospiz-balthasar.de/unser-haus/kinderhospiz.html; abgerufen am 20.03.2015

Kinderpalliativzentrum Göttingen (2017): Ambulante Palliativversorgung http://www.kinderpalliativmedizin-goettingen.de; abgerufen am 14.01.2017

Kinderpalliativzentrum Datteln (2017): Fort- und Weiterbildung https://www.kinderpalliativzentrum.de/de/fort-und-weiterbildung; abgerufen am 21.03.2017

Online- Lexikon für Psychologie und Pädagogik (2017): Begriffsdefinition Abstraktionsniveau

http://lexikon.stangl.eu/6911/abstraktionsniveau/; abgerufen am 14.01.2017

Palliativ Portal (2015): Geschichte der Palliativmedizin http://www.palliativ-portal.de/Historie; abgerufen am 16.03.2015

Pons (2015): Wortbedeutung palliare http://de.pons.com/übersetzung/latein-deutsch/palliare; abgerufen am16.03.2015

Schweizerische Palliativgesellschaft (2015): Die Geschichte der Palliative Care https://www.palliative.ch/de/palliative-care/die-geschichte-der-palliative-care/; abgerufen am 16.03.2015

Statistisches Bundesamt (2015): Zahlen und Fakten: Kinder mit lebenslimitierenden Erkrankungen https://www.destatis.de/DE/ZahlenFakten/GesellschaftStaat/Bevoelkerung/Bevoelkerungsstand/Tabellen_/lrbev01.html; abgerufen am 16.05.2016 
Weiterbildungsordnung Palliativmedizin 2003: Weiterbildungsordnung Palliativmedizin von 2003 in der Fassung vom 25.06.11. Akademie für Palliativmedizin, Palliativpflege und Hospizarbeit Nordhessen e.V.

http//www.apph-nordhessen.de/satzung.htm; abgerufen am 14.01.2017

WHO (1998): Definition von Palliativmedizin

http://www.who.int./cancer/palliative/definition/print.html;

abgerufen am15.03.2015

WHO (2002a): Definition von Palliativmedizin

https://www.dgpalliativmedizin.de/images/stories/WHO_Definition_2002_Pallia-

tive_Care_englisch-deutsch.pdf;

abgerufen am 02.02.2017

WHO (2002b): Definition von Palliativmedizin

http://www.who.int./cancer/palliative/definition/en/;

abgerufen am 02.02.2017

Wikipedia (2015): Transkription (Sozialwissenschaften)

https://de.wikipedia.org/wiki/Transkription_(Sozialwissenschaften);

abgerufen am 21.03.2015

Zernikow B: Spezialisierte Ambulante Palliatativ-Versorgung (SAPV) von Kindern und Jugendlichen! Zuhause und darüber hinaus? Vortrag im Rahmen der Elternbegegnungstagung des Vereins Intensivkinder zuhause e.V., Hamburg 2014

https://www.intensivkinder.de/dld/berichte/veranstaltungen/Vortrag_Dr_Zerni-

kow_EBT_2014;

abgerufen am 31.01.2017 


\section{Danksagung}

Zunächst möchte ich mich bei Prof. Dr. med. Friedemann Nauck bedanken, der mir diese Doktorarbeit ermöglicht und mich stets zuverlässig betreut hat. Weiterer Dank gilt Frau Prof. Dr. med. Jutta Gärtner für die wertvolle Kooperation mit der Klinik für Kinder- und Jugendmedizin Göttingen. Besonderer Dank gilt meiner Betreuerin Frau Dr. med. Gesine Benze für ihr persönliches Engagement, ihre Geduld, ihr Vertrauen und ihre vielfältige Unterstützung. Dr. disc. pol. Gabriella Marx, Sonja Owusu-Boakye MA, und Maximiliane Jansky MA möchte ich ebenfalls danken. Mit ihrer Kompetenz und Erfahrung im Bereich der qualitativen Forschung haben sie diese Arbeit begleitet und mich durch ihre fördernde Haltung immer wieder motiviert und ermutigt. Im Weiteren möchte ich mich bei den Teilnehmern der "Forschungswerkstatt" für den konstruktiven Austausch und die wertvollen Anregungen bedanken. Außerdem gilt mein Dank den Teilnehmern der Studie für die Offenheit in den Interviews und ihre Zeit, die ich in Anspruch nehmen durfte. 